\title{
GLOBAL STRONG SOLUTION TO THE DENSITY-DEPENDENT INCOMPRESSIBLE FLOW OF LIQUID CRYSTALS
}

\author{
XIAOLI LI AND DEHUA WANG
}

Abstract. The initial-boundary value problem for the density-dependent incompressible flow of liquid crystals is studied in a three-dimensional bounded smooth domain. For the initial density away from vacuum, the existence and uniqueness are established for both the local strong solution with large initial data and the global strong solution with 'small' data. It is also proved that when the strong solution exists, a weak solution with the same data must be equal to the unique strong solution.

\section{INTRODUCTION}

Liquid crystals are substances that exhibit a phase of matter that has properties between those of a conventional liquid and those of a solid crystal. A liquid crystal may flow like a liquid, but its molecules may be oriented in a crystal-like way. There are many different types of liquid crystal phases, which can be distinguished based on their different optical properties. One of the most common liquid crystal phases is the nematic, where the molecules have no positional order, but they have longrange orientational order. The three-dimensional density-dependent incompressible flow of nematic liquid crystals can be governed by the following system of partial differential equations $([4,12,25,28])$ :

$$
\begin{aligned}
& \partial_{t} \rho+\nabla \cdot(\rho \mathbf{u})=0, \\
& \partial_{t}(\rho \mathbf{u})+\nabla \cdot(\rho \mathbf{u} \otimes \mathbf{u})+\nabla P=\mu \triangle \mathbf{u}-\lambda \nabla \cdot(\nabla \mathbf{d} \odot \nabla \mathbf{d}), \\
& \partial_{t} \mathbf{d}+\mathbf{u} \cdot \nabla \mathbf{d}=\gamma\left(\Delta \mathbf{d}+|\nabla \mathbf{d}|^{2} \mathbf{d}\right), \\
& \nabla \cdot \mathbf{u}=0,
\end{aligned}
$$

where $\rho$ denotes the density, $\mathbf{u} \in \mathbb{R}^{3}$ the velocity, $\mathbf{d} \in \mathbb{S}^{2}$ (the unit sphere in $\mathbb{R}^{3}$ ) the unit-vector field that represents the macroscopic molecular orientations, $P \in \mathbb{R}$ the pressure (including both the hydrostatic part and the induced elastic part from the orientation field arising from the incompressibility $\nabla \cdot \mathbf{u}=0$ ); they all depend on the spatial variable $\mathbf{x} \in \mathbb{R}^{3}$ and the time variable $t>0$. The positive

Received by the editors January 3, 2012 and, in revised form, June 27, 2012.

2010 Mathematics Subject Classification. Primary 35A05, 76A10, 76D03.

Key words and phrases. Liquid crystals, incompressible flow, density-dependent, global strong solution, existence and uniqueness.

The first author's research was supported in part by the National Natural Science Foundation of China under grant 11401036, by the National Natural Science Foundation of China under grants 11271052 and 11471050, by the China Postdoctoral Science Foundation Funded Project under grant 2013T60085, and by the Fundamental Research for the Central Universities No. 2014 RC 0901.

The second author's research was supported in part by the National Science Foundation under grant DMS-0906160, and by the Office of Naval Research under grant N00014-07-1-0668. 
constants $\mu, \lambda, \gamma$ stand for viscosity, the competition between kinetic energy and potential energy, and microscopic elastic relaxation time or the Deborah number for the molecular orientation field, respectively. The term $\lambda \nabla \cdot(\nabla \mathbf{d} \odot \nabla \mathbf{d})$ in the stress tensor represents the anisotropic feature of the system. We set $\mu=\lambda=$ $\gamma=1$ since their exact values do not play any role in our analysis. The symbol $\nabla \mathbf{d} \odot \nabla \mathbf{d}$ denotes a matrix whose $(i, j)$-th entry is $\partial_{x_{i}} \mathbf{d} \cdot \partial_{x_{j}} \mathbf{d}$ for $1 \leq i, j \leq$ 3 , and it is easy to see that $\nabla \mathbf{d} \odot \nabla \mathbf{d}=(\nabla \mathbf{d})^{\top} \nabla \mathbf{d}$, where $(\nabla \mathbf{d})^{\top}$ denotes the transpose of the $3 \times 3$ matrix $\nabla \mathbf{d}$. System (1.1) is a simplified version, but still retains most of the interesting mathematical properties (without destroying the basic nonlinear structure) of the original Ericksen-Leslie model ([14, 15, 18, 19, 25, 27] ) for the hydrodynamics of nematic liquid crystals; see [10,11,28, 34, 39, for more discussions on the relations of the two models. Both the Ericksen-Leslie system and the simplified one (1.1) describe the macroscopic continuum time evolution of liquid crystal materials under the influence of both the velocity and the orientation of crystals which can be derived from the averaging/coarse graining of the directions of rod-like liquid crystal molecules. In particular, there is a force term in the usystem (1.1b) depending on $\mathbf{d}$; the left-hand side of the $\mathbf{d}$-system (1.1c) stands for the kinematic transport by the flow field, while the right-hand side represents the internal relaxation due to the elastic energy. In many situations, the flow velocity field does disturb the alignment of molecules, and in turn a change in the alignment will induce velocity.

We consider the initial-boundary value problem of system (1.1) in a bounded smooth domain $\Omega \subset \mathbb{R}^{3}$ with the initial condition:

$$
\left.(\rho, \mathbf{u}, \mathbf{d})\right|_{t=0}=\left(\rho_{0}, \mathbf{u}_{0}, \mathbf{d}_{0}\right), \quad \mathbf{x} \in \Omega,
$$

and the boundary condition:

$$
\left.\left(\mathbf{u}, \partial_{\nu} \mathbf{d}\right)\right|_{\partial \Omega}=(\mathbf{0}, 0)
$$

where $\rho_{0}: \Omega \rightarrow \mathbb{R}^{+}, \mathbf{u}_{0}: \Omega \rightarrow \mathbb{R}^{3}, \mathbf{d}_{0}: \Omega \rightarrow \mathbb{S}^{2}$ are given with compatibility, and $\nu$ denotes the outer unit-normal vector field on $\partial \Omega$. The boundary condition implies nonslip on the boundary and no contribution to the surface forces from the director field $\mathbf{d}$. The homogeneous Neumann boundary condition on $\mathbf{d}$ makes it mathematically convenient since $\left.\partial_{\nu}(\mathbf{d}-\overline{\mathbf{d}})\right|_{\partial \Omega}=\left.\partial_{\nu} \mathbf{d}\right|_{\partial \Omega}=0$ with a constant vector $\overline{\mathbf{d}} \in \mathbb{S}^{2}$ and this 'no-flux' condition conforms to the homogeneous boundary conditions in [1]. Roughly speaking, (1.1) is a coupling between the incompressible inhomogeneous (density-dependent) Navier-Stokes equations and the transported flow of harmonic maps. In the homogeneous case $\rho \equiv 1$, (1.1) becomes the hydrodynamic flow system of incompressible liquid crystals. In a series of papers [28]-31, Lin and Liu addressed both the regularity and existence of global weak solutions to the Leslie system of variable length, i.e. when the Dirichlet energy

$$
\frac{1}{2} \int_{\Omega}|\nabla \mathbf{d}|^{2} d \mathbf{x}, \quad \mathbf{d}: \Omega \rightarrow \mathbb{S}^{2},
$$

is replaced by the Ginzburg-Landau energy

$$
\int_{\Omega}\left(\frac{1}{2}|\nabla \mathbf{d}|^{2}+\frac{\left(1-|\mathbf{d}|^{2}\right)^{2}}{4 \varepsilon^{2}}\right) d \mathbf{x}(\varepsilon>0), \quad \mathbf{d}: \Omega \rightarrow \mathbb{R}^{3} .
$$

In particular, for any fixed $\varepsilon>0$, they 28 proved the global existence of weak solutions with large initial data under the assumption that $\mathbf{u}_{0} \in L^{2}(\Omega), \mathbf{d}_{0} \in H^{1}(\Omega)$ with $\left.\mathbf{d}_{0}\right|_{\partial \Omega} \in H^{\frac{3}{2}}(\partial \Omega)$ in the two-dimensional and three-dimensional cases. The 
existence and uniqueness of global classical solution were also obtained if $\mathbf{u}_{0} \in$ $H^{1}(\Omega), \mathbf{d}_{0} \in H^{2}(\Omega)$ when the fluid viscosity $\mu$ is large enough. The partial regularity of the weak solution was investigated in [29] (and also in [5, 19, 34]), similarly to the classical theorem by Caffarelli, Kohn, and Nirenburgh [3] on the Navier-Stokes equations that asserts that the one-dimensional parabolic Hausdorff measure of the singular set of any suitable weak solution is zero. With the Ginzburg-Landau penalty function, the global strong and weak solutions to the compressible flow of liquid crystals were obtained in [35, 37, 40. See also [6, 20, 23, 34, 39] for some related discussions. For the incompressible version of system (1.1) with constant density, Lin-Lin-Wang [32] established the existence of global weak solutions that are smooth away from at most finitely many singular times in any bounded smooth domain of $\mathbb{R}^{2}$, and we [26] proved the global existence of a strong solution in a bounded smooth domain of $\mathbb{R}^{3}$. For the compressible version of system (1.1), the one-dimensional classical solution was obtained in [10,11,38, and the blowup criteria of strong solutions were studied in [21,22].

In this paper, we are interested in the existence and uniqueness of global strong solution $(\rho, \mathbf{u}, P, \mathbf{d})$ of (1.1) in $W^{1, r}(\Omega) \times W^{2, q}(\Omega)^{3} \times W^{1, q}(\Omega) \times W^{3, q}(\Omega)^{3}$ with $3<q \leq r \leq \infty$ while assuming in addition that the initial density is bounded away from zero. By a strong solution, we mean a quadruplet $(\rho, \mathbf{u}, P, \mathbf{d})$ satisfying (1.1) almost everywhere with the initial-boundary conditions (1.2)-(1.3). Note that, for a constant vector $\overline{\mathbf{d}} \in \mathbb{S}^{2}, \nabla \mathbf{d}=\nabla(\mathbf{d}-\overline{\mathbf{d}}), \partial_{t} \mathbf{d}=\partial_{t}(\mathbf{d}-\overline{\mathbf{d}}), \Delta \mathbf{d}=\Delta(\mathbf{d}-\overline{\mathbf{d}})$ in $\Omega$, and $\left.\partial_{\nu} \mathbf{d}\right|_{\partial \Omega}=\left.\partial_{\nu}(\mathbf{d}-\overline{\mathbf{d}})\right|_{\partial \Omega}=0$. Our strategy is to consider the following auxiliary problem:

$$
\left\{\begin{array}{l}
\partial_{t} \rho+\mathbf{v} \cdot \nabla \rho=0 \\
\rho \partial_{t} \mathbf{u}-\Delta \mathbf{u}+\nabla P=-\rho \mathbf{v} \cdot \nabla \mathbf{v}-\nabla \cdot\left((\nabla(\mathbf{f}-\overline{\mathbf{d}}))^{\top} \nabla(\mathbf{f}-\overline{\mathbf{d}})\right) \\
\partial_{t}(\mathbf{d}-\overline{\mathbf{d}})-\Delta(\mathbf{d}-\overline{\mathbf{d}})=-\mathbf{v} \cdot \nabla(\mathbf{f}-\overline{\mathbf{d}})+|\nabla(\mathbf{f}-\overline{\mathbf{d}})|^{2}(\mathbf{f}-\overline{\mathbf{d}})+|\nabla(\mathbf{f}-\overline{\mathbf{d}})|^{2} \overline{\mathbf{d}} \\
\nabla \cdot \mathbf{u}=0 \\
\left.(\rho, \mathbf{u}, \mathbf{d}-\overline{\mathbf{d}})\right|_{t=0}=\left(\rho_{0}, \mathbf{u}_{0}, \mathbf{d}_{0}-\overline{\mathbf{d}}\right) \\
\left.\left(\mathbf{u}, \partial_{\nu}(\mathbf{d}-\overline{\mathbf{d}})\right)\right|_{\partial \Omega}=(\mathbf{0}, 0)
\end{array}\right.
$$

for some given vectors $\mathbf{v} \in \mathbb{R}^{3}, \mathbf{f} \in \mathbb{R}^{3}$. One of the motivations for such a strategy is that the continuity equation (1.1a) is the transport equation of $\rho$, (1.1b) is the evolutionary density-dependent incompressible Navier-Stokes equation with the source term $-\lambda \nabla \cdot(\nabla \mathbf{d} \odot \nabla \mathbf{d})$, while (1.1c) is the parabolic system in terms of d. Therefore we can use a result of the transport equation (cf. Proposition 3.1), the maximal regularities of the parabolic equations (cf. Theorem 3.1) and densitydependent Stokes equations (cf. Theorem [3.2). We first use an iteration method based on (1.4) to establish the local existence and uniqueness of the strong solution with general initial data. Then we prove the global existence by establishing some global estimates under the condition that the initial data are small (close to an equilibrium state) in some sense. As system (1.1) contains the Navier-Stokes equations as a subsystem, one cannot expect in general any better results than those for the Navier-Stokes equations. The uniqueness of a global weak solution is always an open problem. We shall prove that when the strong solution exists, all the global weak solutions must be equal to the unique strong solution, which is called the weak-strong uniqueness. Similar results were obtained by Danchin [8] for the density-dependent incompressible viscous fluids in a bounded domain of $\mathbb{R}^{2}$ with 
$C^{2+\varepsilon}$ boundary. We shall establish our results in the spirit of 8 , while developing new estimates for the crystal orientation field. Due to the particular structure of the equations for the velocity, especially the strongly nonlinear term $(\nabla \mathbf{d})^{\top} \triangle \mathbf{d}$ in the $\mathbf{u}$-system, it will be necessary to obtain more regularities for the crystal orientation field. By developing more novel and subtle estimates, we will be able to finally establish the global existence of strong solution and weak-strong uniqueness for the initial-boundary value problem (1.1)-(1.3) of the density-dependent incompressible flow of liquid crystals. The results of this paper generalize our early results in 26$]$ for the incompressible case with constant density. The analysis in this paper is much more difficult and complicated than that in [26] due to the appearance of nonconstant density.

The rest of the paper is organized as follows. In Section 2, we state our main results on local and global existence of the strong solution, as well as the weak-strong uniqueness. In Section 3, we recall a standard result for the transport equation, the maximal regularities for the nonhomogeneous nonstationary Stokes operator and the parabolic operator, and also some $L^{\infty}$ estimates in the spatial variable. In Section 4, we give the proof of the local existence. In Section 5, we prove the global existence. Finally, in Section 6, we show the weak-strong uniqueness.

\section{MAin Results}

In this section, we state our main results. If $k>0$ is an integer and $p \geq 1$, we denote by $W^{k, p}$ the set of functions in $L^{p}(\Omega)$ whose derivatives of up to order $k$ belong to $L^{p}(\Omega)$. For $T>0$ and a function space $X$, denote by $L^{p}(0, T ; X)$ the set of Bochner measurable $X$-valued time dependent functions $f$ such that $t \rightarrow\|f\|_{X}$ belongs to $L^{p}(0, T)$, and the corresponding Lebesgue norm is denoted by $\|\cdot\|_{L_{T}^{p}(X)}$. We will consider the solutions in the functional spaces defined below.

Definition 2.1. For $T>0$ and $1<p, q, r<\infty$, we denote by $M_{T}^{p, q, r}$ the set of quadruplets $(\rho, \mathbf{u}, P, \mathbf{d})$ such that

$$
\begin{gathered}
\mathbf{u} \in C\left([0, T] ; D_{A_{q}}^{1-\frac{1}{p}, p}\right) \cap L^{p}\left(0, T ; W^{2, q}(\Omega) \cap W_{0}^{1, q}(\Omega)\right), \\
\partial_{t} \mathbf{u} \in L^{p}\left(0, T ; L^{q}(\Omega)\right), \nabla \cdot \mathbf{u}=0 ; \\
\mathbf{d} \in C\left([0, T] ; B_{q, p}^{3\left(1-\frac{1}{p}\right)}\right) \cap L^{p}\left(0, T ; W^{3, q}(\Omega)\right), \quad \partial_{t} \mathbf{d} \in L^{p}\left(0, T ; L^{q}(\Omega)\right) ; \\
\rho \in C\left([0, T] ; W^{1, r}(\Omega)\right) ; \quad P \in L^{p}\left(0, T ; W^{1, q}(\Omega)\right) \text { and } \int_{\Omega} P d \mathbf{x}=0 .
\end{gathered}
$$

If $r=\infty$, then $\rho$ belongs to $L^{\infty}\left(0, T ; W^{1, \infty}(\Omega)\right) \cap C(\Omega \times[0, T])$ instead of $C\left([0, T] ; W^{1, \infty}(\Omega)\right)$. The corresponding norm is denoted by $\|\cdot\|_{M_{T}^{p, q, r}}$.

We notice that the condition $\int_{\Omega} P d \mathbf{x}=0$ in Definition 2.1 holds automatically if we replace $P$ by

$$
P-\frac{1}{|\Omega|} \int_{\Omega} P d \mathbf{x}
$$

in (1.1). Also, in the above definition, the space $D_{A_{q}}^{1-\frac{1}{p}, p}$ stands for some fractional domain of the Stokes operator in $L^{q}$ (cf. Section 2.3 in [8]). Roughly, the vectorfields of $D_{A_{q}}^{1-\frac{1}{p}, p}$ are vectors which have $2-\frac{2}{p}$ derivatives in $L^{q}$, are divergence-free, 
and vanish on $\partial \Omega$. The Besov space (for definition, see [2]) $B_{q, p}^{3\left(1-\frac{1}{p}\right)}$ can be regarded as the interpolation space between $L^{q}$ and $W^{3, q}$, that is,

$$
B_{q, p}^{3\left(1-\frac{1}{p}\right)}=\left(L^{q}, W^{3, q}\right)_{1-\frac{1}{p}, p}
$$

Moreover, we note that $B_{q, p}^{3\left(1-\frac{1}{p}\right)} \hookrightarrow W^{1, q}$ if $p \geq \frac{3}{2}$. By the embedding $W^{1, q} \hookrightarrow$ $L^{\infty}$ as $q>3$, one has $B_{q, p}^{3\left(1-\frac{1}{p}\right)} \hookrightarrow L^{\infty}$, which will be used repeatedly in this paper.

Remark 2.1. System (1.1) has the following scaling property. If $\rho(\mathbf{x}, t), \mathbf{u}(\mathbf{x}, t)$, $P(\mathbf{x}, t)$ and $\mathbf{d}(\mathbf{x}, t)$ solve (1.1), then for each $l>0, \rho_{l}=\rho\left(l \mathbf{x}, l^{2} t\right), \mathbf{u}_{l}=l \mathbf{u}\left(l \mathbf{x}, l^{2} t\right)$, $P_{l}=l^{2} P\left(l \mathbf{x}, l^{2} t\right)$ and $\mathbf{d}_{l}=\mathbf{d}\left(l \mathbf{x}, l^{2} t\right)$ also solve (1.1). Motivated by this scaling invariance observation, the regularity of the director field $\mathbf{d}$ is one order higher than that of the velocity $\mathbf{u}$. A well-known global existence result was obtained in [16] for the compressible Navier-Stokes equations in the homogeneous Sobolev space $\dot{H}^{\frac{1}{2}}$; this reminds us of a function space like $\dot{H}^{\frac{3}{2}}$ for $\mathbf{d}$. However, bounds of the director field $\mathbf{d}$ in $\dot{H}^{\frac{3}{2}}$ cannot imply the $L^{\infty}$ bound of $\mathbf{d}$. To overcome this difficulty, inspired by [7] for the compressible Navier-Stokes equations, it seems more natural to work the liquid crystal equation in the framework of homogeneous Besov space $B_{q, p}^{3\left(1-\frac{1}{p}\right)}$ which is continuously embedded into $L^{\infty}$. Taking the definition of the strong solution into account, we choose the $L^{q}$ setting instead of an $L^{2}$ one so that (1.1) can be satisfied almost everywhere.

The local existence will be shown by using an iterative method, and if the initial data are sufficiently 'small' in some suitable function spaces, which means that the initial data $\left(\mathbf{u}_{0}, \mathbf{d}_{0}\right)$ are close to an equilibrium state $(\mathbf{0}, \overline{\mathbf{d}})$ with $\overline{\mathbf{d}} \in \mathbb{S}^{2}$ being a constant unit vector, the solution is indeed global in time. More precisely, our existence result reads as follows.

Theorem 2.1. Let $\Omega$ be a bounded smooth domain of $\mathbb{R}^{3}$. Assume that $\frac{3}{2} \leq$ $p<\infty, 3<q \leq r \leq \infty$, and $\rho_{0} \in W^{1, r}(\Omega)$ with $\rho_{0} \geq \check{\rho}$ for some $\check{\rho}>0$, $\mathbf{u}_{0} \in D_{A_{q}}^{1-\frac{1}{p}, p}, \mathbf{d}_{0}-\overline{\mathbf{d}} \in B_{q, p}^{3\left(1-\frac{1}{p}\right)}, \overline{\mathbf{d}} \in \mathbb{S}^{2}$ is an arbitrary constant vector. Then,

(1) there exists $T_{0}>0$ such that system (1.1) with the initial-boundary conditions (1.2) -(1.3) has a unique strong solution $(\rho, \mathbf{u}, P, \mathbf{d})$ such that $(\rho, \mathbf{u}, P$, $\mathbf{d}-\overline{\mathbf{d}}) \in M_{T_{0}}^{p, q, r}$ with $0<\check{\rho} \leq \rho$ and $|\mathbf{d}|=1$ on $\Omega \times\left[0, T_{0}\right]$.

(2) Moreover, there exist a constant $\nu>0$ depending on $p, q, r, \check{\rho}, \mu, \lambda, \gamma, \Omega$ and a constant $\delta>0$ depending only on $p, q, r$ such that if

$$
\left\|\mathbf{u}_{0}\right\|_{D_{A_{q}}^{1-\frac{1}{p}, p}}+\left\|\mathbf{d}_{0}-\overline{\mathbf{d}}\right\|_{B_{q, p}^{3\left(1-\frac{1}{p}\right)}} \leq \frac{\nu}{\left(1+\left\|\rho_{0}\right\|_{W^{1, r}}\right)^{\delta}}
$$

holds for the initial data, then the initial-boundary value problem (1.1)-(1.3) has a unique strong solution $(\rho, \mathbf{u}, P, \mathbf{d})$ such that $(\rho, \mathbf{u}, P, \mathbf{d}-\overline{\mathbf{d}}) \in M_{T}^{p, q, r}$ for all $T>0$. Furthermore, denoting by $\lambda_{1}$ the first eigenvalue of the Dirichlet-Laplace operator in $\Omega$, for some constant $C$ depending on $\mu, \lambda$ and $\gamma$, we have the following inequality for all $t \in \mathbb{R}^{+}$:

$$
\|(\sqrt{\rho} \mathbf{u})(t)\|_{L^{2}}+\|\nabla \mathbf{d}(t)\|_{L^{2}} \leq C e^{-\frac{\lambda_{1}}{\hat{\rho}} t}\left(\left\|\sqrt{\rho_{0}} \mathbf{u}_{0}\right\|_{L^{2}}+\left\|\nabla \mathbf{d}_{0}\right\|_{L^{2}}\right)\left(1+\left(\frac{2 \lambda_{1}}{\hat{\rho}} t\right)^{\frac{1}{2}} e^{\frac{\lambda_{1}}{\hat{\rho}} t}\right),
$$


with $\hat{\rho}=\left\|\rho_{0}\right\|_{L^{\infty}}$, and for some positive constant $K$ depending only on $\left\|\rho_{0}\right\|_{W^{1, r},}, p, q, r, \mu, \lambda, \gamma, \check{\rho}$ and $\Omega$,

$$
\|(\rho, \mathbf{u}, P, \mathbf{d}-\overline{\mathbf{d}})\|_{M_{t}^{p, q, r}} \leq K\left(\left\|\mathbf{u}_{0}\right\|_{D_{A_{q}}^{1-\frac{1}{p}, p}}+\left\|\mathbf{d}_{0}-\overline{\mathbf{d}}\right\|_{B_{q, p}^{3\left(1-\frac{1}{p}\right)}}\right) .
$$

Similar to [32], a weak solution to (1.1) with the initial-boundary conditions (1.2)-(1.3) means a quadruplet $(\tilde{\rho}, \tilde{\mathbf{u}}, \Pi, \tilde{\mathbf{d}})$ satisfying system (1.1) in $\Omega \times(0, T)$ for $0<T \leq \infty$ in the sense of distributions, i.e.

$$
\begin{gathered}
\iint_{\Omega \times(0, T)}\left(\tilde{\rho} \partial_{t} \phi+\tilde{\rho} \tilde{\mathbf{u}} \cdot \nabla \phi\right) d \mathbf{x} d t+\int_{\Omega} \rho_{0} \phi(\cdot, 0) d \mathbf{x}=0 \\
-\iint_{\Omega \times(0, T)} \tilde{\rho} \tilde{\mathbf{u}} \cdot\left(\partial_{t} \phi+\tilde{\mathbf{u}} \cdot \nabla \phi\right) d \mathbf{x} d t+\iint_{\Omega \times(0, T)} \nabla \tilde{\mathbf{u}}: \nabla \phi d \mathbf{x} d t \\
=\int_{\Omega} \rho_{0} \mathbf{u}_{0} \cdot \phi(\cdot, 0) d \mathbf{x}+\iint_{\Omega \times(0, T)}(\nabla \mathbf{d} \odot \nabla \mathbf{d}): \nabla \phi d \mathbf{x} d t
\end{gathered}
$$

and

$$
\begin{aligned}
& -\iint_{\Omega \times(0, T)} \tilde{\mathbf{d}} \cdot \partial_{t} \phi d \mathbf{x} d t+\iint_{\Omega \times(0, T)} \tilde{\mathbf{u}} \cdot \nabla \tilde{\mathbf{d}} \cdot \phi d \mathbf{x} d t+\iint_{\Omega \times(0, T)}(\nabla \tilde{\mathbf{d}}):(\nabla \phi) d \mathbf{x} d t \\
& =\int_{\Omega} \mathbf{d}_{0} \cdot \phi(\cdot, 0) d \mathbf{x}+\iint_{\Omega \times(0, T)}|\nabla \tilde{\mathbf{d}}|^{2} \tilde{\mathbf{d}} \cdot \phi d \mathbf{x} d t
\end{aligned}
$$

for all $\phi \in C_{c}^{\infty}(\Omega \times[0, T) ; \mathbb{R})$ or $C_{c}^{\infty}\left(\Omega \times[0, T) ; \mathbb{R}^{3}\right)$. Moreover, $(\tilde{\mathbf{u}}, \tilde{\mathbf{d}})$ satisfies (1.3) in the sense of trace. In this weak formulation, the pressure $\Pi$ can be determined as in the Navier-Stokes equations (see [17]).

Next, we will give a uniqueness result. Suppose

$$
\begin{gathered}
\tilde{\rho} \in L^{\infty}(\Omega \times[0, T]) \cap C\left(0, T ; L^{p}(\Omega)\right), \forall p \geq 1, \\
\tilde{\mathbf{u}} \in\left(L^{2, \infty}(\Omega \times[0, T]) \cap W_{2}^{1,0}\left(\Omega_{T}\right)\right)^{3}, \tilde{\rho}|\tilde{\mathbf{u}}|^{2} \in L^{\infty}\left(0, T ; L^{1}(\Omega)\right), \\
\tilde{\mathbf{d}} \in\left(L^{\infty}\left([0, T], H^{1}(\Omega)\right) \cap L^{2}\left([0, T], H^{2}(\Omega)\right)\right)^{3},|\tilde{\mathbf{d}}|=1, \nabla \Pi \in\left(L^{\frac{4}{3}}\left(0, T ; L^{\frac{6}{5}}(\Omega)\right)\right)^{3}
\end{gathered}
$$

(for all $T \in(0, \infty)$ ) is a global weak solution to (1.1)-(1.3). Then, we have the following energy inequality (cf. [32, Section 5, for the two-dimensional homogeneous case):

$$
\begin{aligned}
& \frac{1}{2} \int_{\Omega}\left(\tilde{\rho}(t)|\tilde{\mathbf{u}}(t)|^{2}+|\nabla \tilde{\mathbf{d}}(t)|^{2}\right) d \mathbf{x}+\int_{0}^{t} \int_{\Omega}\left(|\nabla \tilde{\mathbf{u}}|^{2}+\left.\left.|\triangle \tilde{\mathbf{d}}+| \nabla \tilde{\mathbf{d}}\right|^{2} \tilde{\mathbf{d}}\right|^{2}\right) d \mathbf{x} d \tau \\
& \leq \frac{1}{2} \int_{\Omega}\left(\rho_{0}\left|\mathbf{u}_{0}\right|^{2}+\left|\nabla \mathbf{d}_{0}\right|^{2}\right) d \mathbf{x}
\end{aligned}
$$

for all $t \in(0, \infty)$.

However, as for the standard Navier-Stokes equations, the question of uniqueness in the above class of solutions remains open. For the same initial-boundary conditions, the relation between weak solutions and strong solutions can be formulated as:

Theorem 2.2. Let $\Omega, p, q, r$ be as in Theorem 2.1 and $\rho_{0}, \mathbf{u}_{0}, \mathbf{d}_{0}$ satisfy the assumptions of Theorem 2.1. Then any weak solution to (1.1)-(1.3) in the above class is unique and indeed is equal to its unique strong solution. 
Usually, the uniqueness in the above theorem is called weak-strong uniqueness. For similar results on the compressible Navier-Stokes equation, we refer the reader to 13,33 .

\section{Maximal Regularity}

In this section, we recall a quite standard result for the transport equation and the maximal regularities for the parabolic operator and the nonhomogeneous nonstationary Stokes operator, and prove some $L^{\infty}$ and $L^{r}$ estimates in the spatial variable as well.

For $T>0,1<p, q<\infty$, denote

$$
\mathcal{W}_{q, p}(0, T):=\left(W^{1, p}\left(0, T ; L^{q}(\Omega)\right)\right)^{3} \cap\left(L^{p}\left(0, T ; W^{3, q}(\Omega)\right)\right)^{3} .
$$

Throughout this paper, $C$ stands for a generic positive constant.

We first recall a result for the transport equation (cf. Proposition 3.1 in [8]):

Proposition 3.1. Let $\Omega$ be a Lipschitz domain of $\mathbb{R}^{3}$ and $\mathbf{v} \in\left(L^{1}(0, T ; \text { Lip })\right)^{3}$ be a solenoidal vector-field such that $\mathbf{v} \cdot \mathbf{n}=0$ on $\partial \Omega$. Let $\rho_{0} \in W^{1, r}(\Omega)$ with $r \in[1, \infty]$. Then the system

$$
\left\{\begin{array}{l}
\partial_{t} \rho+\mathbf{v} \cdot \nabla \rho=0 \\
\left.\rho\right|_{t=0}=\rho_{0}
\end{array}\right.
$$

has a unique solution in $L^{\infty}\left(0, T ; W^{1, \infty}(\Omega)\right) \cap C\left([0, T] ; \cap_{q<\infty} W^{1, q}(\Omega)\right)$ if $r=\infty$, and in $C\left([0, T] ; W^{1, r}(\Omega)\right)$ if $r<\infty$. Moreover, the following estimate holds:

$$
\|\rho(t)\|_{W^{1, r}} \leq e^{\int_{0}^{t}\|\nabla \mathbf{v}(\tau)\|_{L^{\infty} d \tau}}\left\|\rho_{0}\right\|_{W^{1, r}}, \quad t \in[0, T] .
$$

If in addition $\rho$ belongs to $L^{p}(\Omega)$ for some $p \in[1, \infty]$, then

$$
\|\rho(t)\|_{L^{p}}=\left\|\rho_{0}\right\|_{L^{p}}, \quad t \in[0, T] .
$$

We first recall the maximal regularity for the parabolic operator (cf. Theorem 4.10.7 and Remark 4.10.9 in [1]):

Theorem 3.1. Given $1<p, q<\infty, \omega_{0} \in B_{q, p}^{3\left(1-\frac{1}{p}\right)}$ and $f \in\left(L^{p}\left(0, T ; L^{q}\left(\mathbb{R}^{3}\right)\right)\right)^{3}$, the Cauchy problem

$$
\left\{\begin{array}{l}
\omega_{t}-\Delta \omega=f \\
\left.\omega\right|_{t=0}=\omega_{0}
\end{array}\right.
$$

has a unique solution $\omega \in \mathcal{W}_{q, p}(0, T)$, and

$$
\|\omega\|_{\mathcal{W}_{q, p}(0, T)} \leq C\left(\|f\|_{L_{T}^{p}\left(L^{q}\right)}+\left\|\omega_{0}\right\|_{B_{q, p}^{3\left(1-\frac{1}{p}\right)}}\right),
$$

where $C$ is independent of $\omega_{0}, f$ and $T$. Moreover, there exists a positive constant $c_{0}$ independent of $f$ and $T$ such that

$$
\|\omega\|_{\mathcal{W}_{q, p}(0, T)} \geq c_{0} \sup _{t \in(0, T)}\|\omega(t)\|_{B_{q, p}^{3\left(1-\frac{1}{p}\right)}} .
$$

Now we recall the existence theorem (cf. Theorem 3.7 in [8]) for the linear system

$$
\left\{\begin{array}{l}
\rho \partial_{t} \mathbf{u}-\mu \triangle \mathbf{u}+\nabla P=f, \quad \int_{\Omega} P d \mathbf{x}=0 \\
\nabla \cdot \mathbf{u}=0, \\
\left.\mathbf{u}\right|_{t=0}=\mathbf{u}_{0},\left.\quad \mathbf{u}\right|_{\partial \Omega}=0 .
\end{array}\right.
$$


Theorem 3.2. Let $\Omega \subset \mathbb{R}^{3}$ be a bounded domain with $C^{2+\varepsilon}$ boundary, $1<p<\infty$ and $3<q \leq r \leq \infty$. Let $\mathbf{u}_{0} \in D_{A_{q}}^{1-\frac{1}{p}, p}$ and $f \in\left(L^{p}\left(0, T ; L^{q}(\Omega)\right)\right)^{3}$. Assume that the density $\rho$ satisfies

$$
0<\check{\rho} \leq \rho(\mathbf{x}, t) \leq \hat{\rho}<\infty, \quad(\mathbf{x}, t) \in \Omega \times(0, T),
$$

and for some $\beta \in(0,1]$,

$$
\rho \in L^{\infty}\left(0, T ; W^{1, r}(\Omega)\right) \cap C^{\beta}\left([0, T] ; L^{\infty}(\Omega)\right) .
$$

Then the system (3.1) has a unique solution $(\mathbf{u}, P)$ such that

$$
\begin{gathered}
\mathbf{u} \in C\left([0, T] ; D_{A_{q}}^{1-\frac{1}{p}, p}\right) \cap\left(L^{p}\left(0, T ; W^{2, q}(\Omega) \cap W_{0}^{1, q}(\Omega)\right)\right)^{3}, \\
\partial_{t} \mathbf{u} \in\left(L^{p}\left(0, T ; L^{q}(\Omega)\right)\right)^{3},
\end{gathered}
$$

and

$$
P \in L^{p}\left(0, T ; W^{1, q}(\Omega)\right) .
$$

Moreover, there exists some constant $C$ depending on $p, q, r$ and $\Omega$ such that for all $t \in[0, T]$, the following inequalities hold:

$$
\begin{aligned}
& \check{\rho}^{\frac{1}{p}} \mu^{1-\frac{1}{p}}\|\mathbf{u}(t)\|_{D_{D_{A_{q}}^{1-\frac{1}{p}, p}}+\mu\|\mathbf{u}\|_{L_{t}^{p}\left(W^{2, q}\right)}+\check{\rho}\left\|\partial_{t} \mathbf{u}\right\|_{L_{t}^{p}\left(L^{q}\right)}+\|P\|_{L_{t}^{p}\left(W^{1, q}\right)}} \\
& \leq C \xi_{\rho}^{3} \mathcal{B}_{\rho}^{2+\tilde{\varsigma}}(t) e^{\frac{C \mu t \mathcal{C}_{\rho}(t)}{\check{\rho} d(\Omega)^{2}}}\left(\check{\rho}^{\frac{1}{p}} \mu^{1-\frac{1}{p}}\left\|\mathbf{u}_{0}\right\|_{D_{A_{q}}^{1-\frac{1}{p}, p}}+\|f\|_{L_{t}^{p}\left(L^{q}\right)}\right)
\end{aligned}
$$

and

$$
\begin{aligned}
& \check{\rho}^{\frac{1}{p}} \mu^{1-\frac{1}{p}}\|\mathbf{u}(t)\|_{D_{A_{q}}^{1-\frac{1}{p}, p}}+\left\|\check{\rho} \partial_{t} \mathbf{u}, \mu \nabla^{2} \mathbf{u}, \nabla P\right\|_{L_{t}^{p}\left(L^{q}\right)} \\
& \leq C\left(\xi_{\rho}^{4} \mathcal{B}_{\rho}^{2+\tilde{\varsigma}}(t)\left(\check{\rho}^{\frac{1}{p}} \mu^{1-\frac{1}{p}}\left\|\mathbf{u}_{0}\right\|_{D_{A_{q}}^{1-\frac{1}{p}, p}}+\|f\|_{L_{t}^{p}\left(L^{q}\right)}\right)+\frac{\xi_{\rho} \mu}{d(\Omega)^{2}} \mathcal{C}_{\rho}(t)\|\mathbf{u}\|_{L_{t}^{p}\left(L^{q}\right)}\right),
\end{aligned}
$$

where $d(\Omega)$ is the diameter of $\Omega, \xi_{\rho}:=\hat{\rho} / \check{\rho}$, and

$$
\begin{gathered}
\mathcal{B}_{\rho}(t):=1+d(\Omega)\left(\check{\rho}^{-1}\|\nabla \rho\|_{L_{t}^{\infty}\left(L^{r}\right)}\right)^{\frac{r}{r-3}} \\
\mathcal{C}_{\rho}(t):=\xi_{\rho}^{\frac{2 q}{q-1}} \mathcal{B}_{\rho}(t)^{r^{*}}+\hat{\rho} \mu^{-1} d(\Omega)^{2} \xi_{\rho}^{1+\frac{1}{\beta}} \mathcal{B}_{\rho}(t)^{\left(1+\frac{1}{\beta}\right)(2+\tilde{\varsigma})} M_{\beta}(t)^{\frac{1}{\beta}},
\end{gathered}
$$

with

$$
M_{\beta}(t):=\check{\rho}^{-1}\|\rho\|_{C_{0, \beta}^{0,0}(\Omega \times[0, t])},
$$

and the exponents $\tilde{\varsigma}, r^{*}$ are numbers determined by $p, q, r$.

Remark 3.1. The reader can also refer to Theorem 3.7 in 8 for more details about Theorem 3.2. We notice that (3.2) and (3.3) do not include the estimate for $\|\mathbf{u}\|_{L_{T}^{p}\left(L^{q}\right)}$. Indeed, since we consider only in a bounded domain $\Omega$, then there exists a constant $C=C(q, d(\Omega))$ such that

$$
\|\mathbf{u}\|_{W^{2, q}} \equiv\left\|\nabla^{2} \mathbf{u}\right\|_{L^{q}}+d(\Omega)^{-1}\|\nabla \mathbf{u}\|_{L^{q}}+d(\Omega)^{-2}\|\mathbf{u}\|_{L^{q}} \leq C\left\|\nabla^{2} \mathbf{u}\right\|_{L^{q}}
$$

whenever $\mathbf{u} \in\left(W^{2, q}(\Omega) \cap W_{0}^{1, q}(\Omega)\right)^{3}$ (cf. Proposition 2.4 in [8] ).

We also have the following two interpolation inequalities for the $L^{\infty}$ and $L^{r}$ estimates in the spatial variable (cf. Lemma 4.1 in [8], also Lemmas 3.1 and 3.3 in [26]) which are useful in our proof. 
Lemma 3.1. Let $1<p, q, r, s<\infty$ satisfy

$$
0<\frac{p}{2}-\frac{3 p}{2 q}<1, \quad \frac{1}{s}=\frac{1}{r}+\frac{1}{q} .
$$

Then the following inequalities hold:

$$
\begin{gathered}
\|\nabla f\|_{L_{T}^{p}\left(L^{\infty}\right)} \leq C T^{\frac{1}{2}-\frac{3}{2 q}}\|f\|_{L_{T}^{\infty}\left(D_{A_{q}}^{1-\frac{1}{p}, p}\right)}^{1-\theta}\|f\|_{L_{T}^{p}\left(W^{2, q}\right)}^{\theta}, \\
\|\nabla f\|_{L_{T}^{p}\left(L^{r}\right)} \leq C T^{\frac{1}{2}-\frac{3}{2 q}}\|f\|_{L_{T}^{\infty}\left(D_{A_{s}}^{1-\frac{1}{p}, p}\right)}^{1-\theta}\|f\|_{L_{T}^{p}\left(W^{2, s}\right)}^{\theta},
\end{gathered}
$$

for some constant $C$ depending only on $\Omega, p, q, r$, and

$$
\frac{1-\theta}{p}=\frac{1}{2}-\frac{3}{2 q} \text {. }
$$

Similarly, we can prove

Lemma 3.2. Let $1<p, q, r, s<\infty$ satisfy

$$
0<\frac{2 p}{3}-\frac{p}{q}<1, \quad \frac{1}{s}=\frac{1}{r}+\frac{1}{q} .
$$

Then

$$
\begin{gathered}
\|\nabla f\|_{L_{T}^{p}\left(L^{\infty}\right)} \leq C T^{\frac{2}{3}-\frac{1}{q}\|f\|^{1-\theta}} \\
\left.\|\nabla f\|_{L_{T}^{p}\left(L^{r}\right)} \leq C T^{\frac{2}{3}-\frac{1}{q}}\|f\|_{L_{T}^{\infty}\left(B_{q, p}^{3\left(1-\frac{1}{p}\right)}\right)}^{1-\theta}\|f\|_{L_{T}^{p}\left(W^{3, p}\right)}^{\theta\left(1-\frac{1}{p}\right)}\right) \\
\|f\|_{L_{T}^{p}\left(W^{3, s}\right)}^{\theta},
\end{gathered}
$$

for some constant $C$ depending only on $\Omega, p, q, r$, and

$$
\frac{1-\theta}{p}=\frac{2}{3}-\frac{1}{q} \text {. }
$$

Proof. The proof is based on the applications of embedding and interpolation results in [2]. First, we notice that, from Theorem 6.4.5 in [2],

$$
\left(B_{\infty, \infty}^{3-\frac{3}{p}-\frac{3}{q}}, B_{\infty, \infty}^{3-\frac{3}{q}}\right)_{\theta, 1}=B_{\infty, 1}^{1} \text { with } \frac{1-\theta}{p}=\frac{2}{3}-\frac{1}{q},
$$

and from the embedding (Theorem 6.2.4 in [2]) $B_{\infty, 1}^{1} \hookrightarrow W^{1, \infty}$, we get

$$
\|\nabla f\|_{L^{\infty}} \leq C\|f\|_{\substack{3-\frac{3}{q} \\ B_{\infty, \infty}}}^{\theta}\|f\|_{B_{\infty, \infty}^{3-\frac{3}{p}-\frac{3}{q}}}^{1-\theta}
$$

We remark that

$$
B_{q, p}^{3\left(1-\frac{1}{p}\right)} \hookrightarrow B_{\infty, \infty}^{3-\frac{3}{p}-\frac{3}{q}}, \quad W^{3, q} \hookrightarrow B_{q, \infty}^{3} \hookrightarrow B_{\infty, \infty}^{3-\frac{3}{q}}
$$

(cf. Theorems 6.5.1 and 6.2.4 in [2]). Therefore, according to (3.4), (3.5) and by Hölder's inequality, we deduce that

$$
\begin{aligned}
& \|\nabla f\|_{L_{T}^{p}\left(L^{\infty}\right)} \leq C\left(\int_{0}^{T}\|f\|_{\substack{3-\frac{3}{p}-\frac{3}{q} \\
B_{\infty, \infty}}}^{p(1-\theta)}\|f\|_{B_{\infty, \infty}^{3-\frac{3}{q}}}^{p \theta} d t\right)^{\frac{1}{p}} \leq C\left(\int_{0}^{T}\|f\|_{B_{q, p}^{3\left(1-\frac{1}{p}\right)}}^{p(1-\theta)}\|f\|_{W^{3, q}}^{p \theta} d t\right)^{\frac{1}{p}} \\
& \leq C T^{\frac{2}{3}-\frac{1}{q}}\|f\|_{L_{T}^{\infty}\left(B_{q, p}^{3\left(1-\frac{1}{p}\right)}\right)}^{1-\theta}\|f\|_{L_{T}^{p}\left(W^{3, q}\right)}^{\theta} .
\end{aligned}
$$

The proof of the second inequality is based on the fact that

$$
B_{r, 1}^{0}=\left(B_{r, p}^{2-\frac{3}{p}-\frac{3}{q}}, B_{r, r}^{2-\frac{3}{q}}\right)_{\theta, 1} \hookrightarrow L^{r} \text { with } \frac{1-\theta}{p}=\frac{2}{3}-\frac{1}{q}
$$


(cf. Theorem 6.4.5 and Theorem 6.2.4 in [2]) and that

$$
W^{2, s} \hookrightarrow B_{r, r}^{2-\frac{3}{q}}, \quad B_{s, p}^{2-\frac{3}{p}} \hookrightarrow B_{r, p}^{2-\frac{3}{p}-\frac{3}{q}}
$$

(cf. Theorems 6.2.4 and 6.5.1 in [2]). In fact, by Hölder's inequality, we have

$$
\begin{aligned}
\|\nabla f\|_{L_{T}^{p}\left(L^{r}\right)} & \leq C\left(\int_{0}^{T}\|\nabla f\|_{B_{r, p}^{2-\frac{3}{p}-\frac{3}{q}}}^{p(1-\theta)}\|\nabla f\|_{B_{r, r}^{2-\frac{3}{q}}}^{p \theta} d t\right)^{\frac{1}{p}} \\
& \leq C\left(\int_{0}^{T}\|\nabla f\|_{B_{s, p}^{2-\frac{3}{p}}}^{p(1-\theta)}\|\nabla f\|_{W^{2, s}}^{p \theta} d t\right)^{\frac{1}{p}} \\
& \leq C T^{\frac{2}{3}-\frac{1}{q}\|f\|_{L_{T}^{\infty}\left(B_{s, p}^{3\left(1-\frac{1}{p}\right)}\right)}^{1-\theta}\|f\|_{L_{T}^{p}\left(W^{3, s}\right)}^{\theta} .}
\end{aligned}
$$

Lemma 3.3. Let $1<p, q, r, s<\infty$ satisfy

$$
0<\frac{p}{3}-\frac{p}{q}<1, \quad \frac{1}{s}=\frac{1}{r}+\frac{1}{q} .
$$

Then

$$
\begin{gathered}
\left\|\nabla^{2} f\right\|_{L_{T}^{p}\left(L^{\infty}\right)} \leq C T^{\frac{1}{3}-\frac{1}{q}}\|f\|_{L_{T}^{\infty}\left(B_{q, p}^{3\left(1-\frac{1}{p}\right)}\right)}^{1-\theta}\|f\|_{L_{T}^{p}\left(W^{3, q}\right)}^{\theta}, \\
\left\|\nabla^{2} f\right\|_{L_{T}^{p}\left(L^{r}\right)} \leq C T^{\frac{1}{3}-\frac{1}{q}\|f\|_{L_{T}^{\infty}\left(B_{s, p}^{3\left(1-\frac{1}{p}\right)}\right)}^{1-\theta}\|f\|_{L_{T}^{p}\left(W^{3, s}\right)}^{\theta},}
\end{gathered}
$$

for some constant $C$ depending only on $\Omega, p, q, r$, and

$$
\frac{1-\theta}{p}=\frac{1}{3}-\frac{1}{q} \text {. }
$$

Proof. First, we notice that (cf. Theorem 6.4.5 in [2])

$$
\left(B_{\infty, \infty}^{2-\frac{3}{p}-\frac{3}{q}}, B_{\infty, \infty}^{2-\frac{3}{q}}\right)_{\theta, 1}=B_{\infty, 1}^{1} \text { with } \frac{1-\theta}{p}=\frac{1}{3}-\frac{1}{q} .
$$

Hence,

$$
\left\|\nabla^{2} f\right\|_{L^{\infty}} \leq C\|\nabla f\|_{W^{1, \infty}} \leq C\|\nabla f\|_{B_{\infty, 1}^{1}} \leq C\|\nabla f\|_{\substack{B_{\infty, \infty}^{2-\frac{3}{q}} \\ \theta}}\|\nabla f\|_{\substack{B_{\infty, \infty}^{2-\theta} \\ 2-\frac{3}{q}}}^{1-\theta} .
$$

We remark that (cf. Theorems 6.2.4 and 6.5.1 in [2])

$$
B_{q, p}^{3\left(1-\frac{1}{p}\right)} \hookrightarrow B_{\infty, \infty}^{3-\frac{3}{p}-\frac{3}{q}}, \quad W^{2, q} \hookrightarrow B_{q, \infty}^{2} \hookrightarrow B_{\infty, \infty}^{2-\frac{3}{q}} .
$$

Thus, according to (3.6) and by applying Hölder's inequality, we deduce that

$$
\begin{aligned}
\left\|\nabla^{2} f\right\|_{L_{T}^{p}\left(L^{\infty}\right)} & \leq C\left(\int_{0}^{T}\|\nabla f\|_{B_{\infty, \infty}^{2-\frac{3}{q}}}^{p \theta}\|\nabla f\|_{B_{\infty, \infty}^{2-\frac{3}{p}-\frac{3}{q}}}^{p(1-\theta)} d t\right)^{\frac{1}{p}} \\
& \leq C\left(\int_{0}^{T}\|f\|_{W^{3, q}}^{p \theta}\|f\|_{B_{q, p}^{3\left(1-\frac{1}{p}\right)}}^{p(1-\theta)} d t\right)^{\frac{1}{p}} \\
& \leq C T^{\frac{1}{3}-\frac{1}{q}\|f\|_{L_{T}^{\infty}\left(B_{q, p}^{3\left(1-\frac{1}{p}\right)}\right)}^{1-\theta}\|f\|_{L_{T}^{p}\left(W^{3, q}\right)}^{\theta} .}
\end{aligned}
$$


The proof of the second inequality is based on the fact that

$$
B_{r, 1}^{0}=\left(B_{r, p}^{1-\frac{3}{p}-\frac{3}{q}}, B_{r, r}^{1-\frac{3}{q}}\right)_{\theta, 1} \hookrightarrow L^{r} \text { with } \frac{1-\theta}{p}=\frac{1}{3}-\frac{1}{q}
$$

(cf. Theorem 6.4.5 and Theorem 6.2.4 in [2]) and that

$$
W^{1, s} \hookrightarrow B_{r, r}^{1-\frac{3}{q}}, \quad B_{s, p}^{1-\frac{3}{p}} \hookrightarrow B_{r, p}^{1-\frac{3}{p}-\frac{3}{q}}
$$

(cf. Theorems 6.2.4 and 6.5.1 in [2]). In fact, by Hölder's inequality, we have

$$
\begin{aligned}
\|\nabla f\|_{L_{T}^{p}\left(L^{r}\right)} & \leq C\left(\int_{0}^{T}\|\nabla f\|_{B_{r, p}^{1-\frac{3}{p}-\frac{3}{q}}}^{p(1-\theta)}\|\nabla f\|_{B_{r, r}^{1-\frac{3}{q}}}^{p \theta} d t\right)^{\frac{1}{p}} \\
& \leq C\left(\int_{0}^{T}\|\nabla f\|_{B_{s, p}^{1-\frac{3}{p}}}^{p(1-\theta)}\|\nabla f\|_{W^{1, s}}^{p \theta} d t\right)^{\frac{1}{p}} \\
& \leq C T^{\frac{1}{3}-\frac{1}{q}\|f\|_{L_{T}^{\infty}\left(B_{s, p}^{2-\frac{3}{p}}\right)}^{1-\theta}\|f\|_{L_{T}^{p}\left(W^{2, s}\right)}^{\theta} .}
\end{aligned}
$$

\section{LOCAL EXISTENCE}

In this section, we prove the existence and uniqueness of the strong solution on a short time interval, i.e. the local strong solution in Theorem 2.1. The proof will be divided into several steps, including constructing the approximate solutions by iteration, obtaining the uniform estimates, and showing the convergence, consistency and uniqueness.

4.1. Construction of approximate solutions. We initialize the construction of approximate solutions by setting $\rho^{0}:=\rho_{0}, \mathbf{u}^{0}:=\mathbf{u}_{0}$ and $\mathbf{d}^{0}:=\mathbf{d}_{0}$. Given $\left(\rho^{n}, \mathbf{u}^{n}, P^{n}, \mathbf{d}^{n}-\overline{\mathbf{d}}\right)$ with a constant vector $\overline{\mathbf{d}} \in \mathbb{S}^{2}$, Proposition 3.1, Theorem 3.1 and Theorem 3.2 enable us to define respectively $\rho^{n+1}(\mathbf{x}, t)$ as the (global) solution of the transport equation

$$
\left\{\begin{array}{l}
\partial_{t} \rho^{n+1}+\mathbf{u}^{n} \cdot \nabla \rho^{n+1}=0, \\
\left.\rho^{n+1}\right|_{t=0}=\rho_{0},
\end{array}\right.
$$

$\mathbf{d}^{n+1}(\mathbf{x}, t)-\overline{\mathbf{d}}$ as the (global) solution of

$$
\left\{\begin{array}{l}
\partial_{t}\left(\mathbf{d}^{n+1}-\overline{\mathbf{d}}\right)-\Delta\left(\mathbf{d}^{n+1}-\overline{\mathbf{d}}\right) \\
\quad=-\mathbf{u}^{n} \cdot \nabla\left(\mathbf{d}^{n}-\overline{\mathbf{d}}\right)+\left|\nabla\left(\mathbf{d}^{n}-\overline{\mathbf{d}}\right)\right|^{2}\left(\mathbf{d}^{n}-\overline{\mathbf{d}}\right)+\left|\nabla\left(\mathbf{d}^{n}-\overline{\mathbf{d}}\right)\right|^{2} \overline{\mathbf{d}} \\
\mathbf{d}^{n+1}-\left.\overline{\mathbf{d}}\right|_{t=0}=\mathbf{d}_{0}-\overline{\mathbf{d}},\left.\quad \partial_{\nu}\left(\mathbf{d}^{n+1}-\overline{\mathbf{d}}\right)\right|_{\partial \Omega}=0
\end{array}\right.
$$

and $\left(\mathbf{u}^{n+1}(\mathbf{x}, t), P^{n+1}(\mathbf{x}, t)\right)$ as the (global) solution of

$$
\left\{\begin{array}{l}
\rho^{n+1} \partial_{t} \mathbf{u}^{n+1}-\Delta \mathbf{u}^{n+1}+\nabla P^{n+1} \\
\quad=-\rho^{n+1} \mathbf{u}^{n} \cdot \nabla \mathbf{u}^{n}-\nabla \cdot\left(\left(\nabla\left(\mathbf{d}^{n}-\overline{\mathbf{d}}\right)\right)^{\top} \nabla\left(\mathbf{d}^{n}-\overline{\mathbf{d}}\right)\right), \\
\nabla \cdot \mathbf{u}^{n+1}=0, \quad \int_{\Omega} P^{n+1} d \mathbf{x}=0 \\
\left.\mathbf{u}^{n+1}\right|_{t=0}=\mathbf{u}_{0},\left.\quad \mathbf{u}^{n+1}\right|_{\partial \Omega}=0
\end{array}\right.
$$

An argument by induction yields a sequence $\left\{\left(\rho^{n}, \mathbf{u}^{n}, P^{n}, \mathbf{d}^{n}-\overline{\mathbf{d}}\right)\right\}_{n \in \mathbb{N}} \subset M_{T}^{p, q, r}$ for all $T>0$. 
4.2. Uniform estimate for some small fixed time $T_{*}$. We aim at finding a positive time $T_{*}$ independent of $n$ for which $\left\{\left(\rho^{n}, \mathbf{u}^{n}, P^{n}, \mathbf{d}^{n}-\overline{\mathbf{d}}\right)\right\}_{n \in \mathbb{N}}$ is uniformly bounded in the space $M_{T_{*}}^{p, q, r}$.

First we control $\rho^{n+1}$ and its derivative in the time variable in terms of $\mathbf{u}_{n}$ and $\nabla \mathbf{u}_{n}$ in spaces $W^{1, r}(\Omega)$ and $L^{s}(\Omega)$ with $s$ (depending on $q, r$ ) to be specified below. Applying Proposition 3.1 to (4.1), we get

$$
\left\|\rho^{n+1}(t)\right\|_{W^{1, r}} \leq e^{\int_{0}^{t}\left\|\nabla \mathbf{u}^{n}(\tau)\right\|_{L} \infty d \tau}\left\|\rho_{0}\right\|_{W^{1, r}}
$$

and

$$
\min _{\mathbf{x} \in \bar{\Omega}} \rho^{n+1}(\mathbf{x}, t)=\check{\rho}:=\min _{\mathbf{x} \in \bar{\Omega}} \rho_{0}(\mathbf{x}) \text { and } \max _{\mathbf{x} \in \bar{\Omega}} \rho^{n+1}(\mathbf{x}, t)=\hat{\rho}:=\max _{\mathbf{x} \in \bar{\Omega}} \rho_{0}(\mathbf{x}) .
$$

Since $\partial_{t} \rho^{n+1}=-\mathbf{u}^{n} \cdot \nabla \rho^{n+1}$, then by Hölder's inequality, we have

$$
\partial_{t} \rho^{n+1} \in L_{\mathrm{loc}}^{\infty}\left(\mathbb{R}^{+} ; L^{s}(\Omega)\right)
$$

with $s=\frac{q r}{q+r}(s=q$ if $r=\infty)$, and for $t \geq 0$,

$$
\left\|\partial_{t} \rho^{n+1}\right\|_{L_{t}^{\infty}\left(L^{s}\right)} \leq\left\|\mathbf{u}^{n}\right\|_{L_{t}^{\infty}\left(L^{q}\right)}\left\|\nabla \rho^{n+1}\right\|_{L_{t}^{\infty}\left(L^{r}\right)} .
$$

Then we control $\mathbf{u}^{n+1}$ in $D_{A_{q}}^{1-\frac{1}{p}, p}$ in terms of $\rho^{n+1}, \mathbf{u}^{n}$ and $\mathbf{d}^{n}-\overline{\mathbf{d}}$. In order to apply Theorem 3.2 to (4.3), we need to prove $\rho^{n+1} \in C^{\beta}\left([0, T] ; L^{\infty}(\Omega)\right)$ for some $\beta \in(0,1]$. Actually, noticing that $\rho^{n+1}$ belongs to $C^{\beta}\left([0, T] ; L^{\infty}(\Omega)\right)$ whenever $\beta \in\left(0, \frac{1-\frac{3}{r}}{1+\frac{3}{q}}\right)$ by interpolation between $L^{\infty}\left(0, T ; W^{1, r}(\Omega)\right)$ and $W^{1, \infty}\left(0, T ; L^{s}(\Omega)\right)$, it holds that

$$
\left\|\rho^{n+1}\right\|_{C_{t}^{\beta}\left(L^{\infty}\right)} \leq C\left(\left\|\rho^{n+1}\right\|_{L_{t}^{\infty}\left(W^{1, r}\right)}+\left\|\partial_{t} \rho^{n+1}\right\|_{L_{t}^{\infty}\left(L^{s}\right)}\right) .
$$

Here we have used Young's inequality.

Hence, applying Theorem 3.2 to (4.3) yields

$$
\begin{aligned}
& \left\|\mathbf{u}^{n+1}(t)\right\|_{D_{A_{q}}^{1-\frac{1}{p}, p}}+\left\|\mathbf{u}^{n+1}\right\|_{L_{t}^{p}\left(W^{2, q}\right)}+\left\|\partial_{t} \mathbf{u}^{n+1}\right\|_{L_{t}^{p}\left(L^{q}\right)}+\left\|P^{n+1}\right\|_{L_{t}^{p}\left(W^{1, q}\right)} \\
& \leq C e^{C t \psi(t)}\left(\left\|\mathbf{u}_{0}\right\|_{D_{A_{q}}^{1-\frac{1}{p}, p}}+\left\|\mathbf{u}^{n} \cdot \nabla \mathbf{u}^{n}+\nabla \cdot\left(\left(\nabla\left(\mathbf{d}^{n}-\overline{\mathbf{d}}\right)\right)^{\top} \nabla\left(\mathbf{d}^{n}-\overline{\mathbf{d}}\right)\right)\right\|_{L_{t}^{p}\left(L^{q}\right)}\right),
\end{aligned}
$$

where

$$
\psi(t)=\left(1+\left\|\rho^{n+1}\right\|_{L_{t}^{\infty}\left(W^{1, r}\right)}\right)^{\gamma_{0}}\left(1+\left\|\rho^{n+1}\right\|_{C_{t}^{\beta}\left(L^{\infty}\right)}^{\frac{1}{\beta}}\right)
$$

for some positive exponent $\gamma_{0}$ depending only on $p, q, r, \beta$ and the constant $C$ depending only on $p, q, r, \check{\rho}, \hat{\rho}, \Omega, \beta$. Using (4.6) and (4.7), we get

$$
\psi(t) \leq C\left(1+\left\|\rho^{n+1}\right\|_{L_{t}^{\infty}\left(W^{1, r}\right)}\right)^{\sigma}\left(1+\left\|\mathbf{u}^{n}\right\|_{L_{t}^{\infty}\left(L^{q}\right)}\right)^{\frac{1}{\beta}},
$$

where $\sigma$ depends only on $p, q, r$ and $\beta$. Therefore,

$$
\begin{aligned}
& \left\|\mathbf{u}^{n+1}(t)\right\|_{D_{A_{q}}^{1-\frac{1}{p}, p}}+\left\|\mathbf{u}^{n+1}\right\|_{L_{t}^{p}\left(W^{2, q}\right)}+\left\|\partial_{t} \mathbf{u}^{n+1}\right\|_{L_{t}^{p}\left(L^{q}\right)}+\left\|P^{n+1}\right\|_{L_{t}^{p}\left(W^{1, q}\right)} \\
& \leq C e^{C t\left(1+\left\|\rho^{n+1}\right\|_{L_{t}^{\infty}\left(W^{1, r}\right)}\right)^{\sigma}\left(1+\left\|\mathbf{u}^{n}\right\|_{L_{t}^{\infty}\left(L^{q}\right)}\right)^{\frac{1}{\beta}}}\left(\left\|\mathbf{u}_{0}\right\|_{D_{A_{q}}^{1-\frac{1}{p}, p}}+\left\|\mathbf{u}^{n} \cdot \nabla \mathbf{u}^{n}\right\|_{L_{t}^{p}\left(L^{q}\right)}\right. \\
& \left.\quad+\left\|\nabla \cdot\left(\left(\nabla\left(\mathbf{d}^{n}-\overline{\mathbf{d}}\right)\right)^{\top} \nabla\left(\mathbf{d}^{n}-\overline{\mathbf{d}}\right)\right)\right\|_{L_{t}^{p}\left(L^{q}\right)}\right) .
\end{aligned}
$$


Finally we control $\mathbf{d}^{n+1}-\overline{\mathbf{d}}$ in Besov space $B_{q, p}^{3\left(1-\frac{1}{p}\right)}$ in terms of $\mathbf{u}^{n}$ and $\mathbf{d}^{n}-\overline{\mathbf{d}}$. Applying Theorem 3.1 to (4.2), we obtain

$$
\begin{aligned}
& \left\|\mathbf{d}^{n+1}(t)-\overline{\mathbf{d}}\right\|_{B_{q, p}^{3\left(1-\frac{1}{p}\right)}}+\left\|\mathbf{d}^{n+1}-\overline{\mathbf{d}}\right\|_{\mathcal{W}_{q, p}(0, t)} \\
& \leq C\left(\left\|\mathbf{d}_{0}-\overline{\mathbf{d}}\right\|_{B_{q, p}^{3\left(1-\frac{1}{p}\right)}}+\|-\mathbf{u}^{n} \cdot \nabla\left(\mathbf{d}^{n}-\overline{\mathbf{d}}\right)+\left|\nabla\left(\mathbf{d}^{n}-\overline{\mathbf{d}}\right)\right|^{2}\left(\mathbf{d}^{n}-\overline{\mathbf{d}}\right)\right. \\
& \left.\quad+\left|\nabla\left(\mathbf{d}^{n}-\overline{\mathbf{d}}\right)\right|^{2} \overline{\mathbf{d}} \|_{L_{t}^{p}\left(L^{q}\right)}\right) .
\end{aligned}
$$

Define

$$
\begin{gathered}
U^{n}(t):=\left\|\mathbf{u}^{n}\right\|_{L_{t}^{\infty}\left(D_{A_{q}}^{1-\frac{1}{p}, p}\right)}+\left\|\mathbf{u}^{n}\right\|_{L_{t}^{p}\left(W^{2, q}\right)}+\left\|\partial_{t} \mathbf{u}^{n}\right\|_{L_{t}^{p}\left(L^{q}\right)} \\
+\left\|\mathbf{d}^{n}-\overline{\mathbf{d}}\right\|_{L_{t}^{\infty}\left(B_{q, p}^{3\left(1-\frac{1}{p}\right)}\right)}+\left\|\mathbf{d}^{n}-\overline{\mathbf{d}}\right\|_{\mathcal{W}_{q, p}(0, t)}, \\
U^{0}=\left\|\mathbf{u}_{0}\right\|_{D_{A_{q}}^{1-\frac{1}{p}, p}}+\left\|\mathbf{d}_{0}-\overline{\mathbf{d}}\right\|_{B_{q, p}^{3\left(1-\frac{1}{p}\right)}}, \\
\varrho^{n}(t):=\left\|\rho^{n}\right\|_{L_{t}^{\infty}\left(W^{1, r}\right)} \text { and } \quad \varrho_{0}:=\left\|\rho_{0}\right\|_{W^{1, r}} .
\end{gathered}
$$

Next we will estimate the right-hand side of (4.4), (4.8) and (4.9) by using Lemmas 3.1]3.3. Since Lemmas 3.1]3.3 are valid for different ranges of $\frac{1}{p}$, we need to establish the estimates for different cases. In fact, the indices are special in Lemmas 3.1 3.3. and $\frac{1}{3}-\frac{1}{q}<\frac{1}{2}-\frac{3}{2 q}<\frac{2}{3}-\frac{1}{q}$ for $q>3$, so we will consider seven different ranges for $\frac{1}{p}$ as follows.

Case 1. $\frac{2}{3}-\frac{1}{q}<\frac{1}{p}$, using Lemmas 3.1 3.3, we get

$$
\varrho^{n+1}(t) \leq e^{t^{1-\frac{1}{p}}\left\|\nabla \mathbf{u}^{n}\right\|_{L_{t}^{p}\left(L^{\infty}\right)}}\left\|\rho_{0}\right\|_{W^{1, r}} \leq \varrho_{0} e^{C t^{\frac{3}{2}-\frac{1}{p}-\frac{3}{2 q}} U^{n}(t)},
$$

$$
\begin{aligned}
& \left\|\mathbf{u}^{n+1}(t)\right\|_{D_{A_{q}}^{1-\frac{1}{p}, p}}+\left\|\mathbf{u}^{n+1}\right\|_{L_{t}^{p}\left(W^{2, q}\right)}+\left\|\partial_{t} \mathbf{u}^{n+1}\right\|_{L_{t}^{p}\left(L^{q}\right)}+\left\|P^{n+1}\right\|_{L_{t}^{p}\left(W^{1, q}\right)} \\
& \leq C e^{C t\left(1+\left\|\rho^{n+1}\right\|_{L_{t}^{\infty}\left(W^{1, r}\right)}\right)^{\sigma}\left(1+\left\|\mathbf{u}^{n}\right\|_{L_{t}^{\infty}\left(L^{q}\right)}\right)^{\frac{1}{\beta}}}\left(\left\|\mathbf{u}_{0}\right\|_{D_{A_{q}}^{1-\frac{1}{p}, p}}+\left\|\mathbf{u}^{n}\right\|_{L_{t}^{\infty}\left(L^{q}\right)}\left\|\nabla \mathbf{u}^{n}\right\|_{L_{t}^{p}\left(L^{\infty}\right)}\right.
\end{aligned}
$$

$$
\left.+\left\|\nabla\left(\mathbf{d}^{n}-\overline{\mathbf{d}}\right)\right\|_{L_{t}^{\infty}\left(L^{q}\right)}\left\|\triangle\left(\mathbf{d}^{n}-\overline{\mathbf{d}}\right)\right\|_{L_{t}^{p}\left(L^{\infty}\right)}\right),
$$

$$
\left\|\mathbf{d}^{n+1}(t)-\overline{\mathbf{d}}\right\|_{B_{q, p}^{3\left(1-\frac{1}{p}\right)}}+\left\|\mathbf{d}^{n+1}-\overline{\mathbf{d}}\right\|_{\mathcal{W}_{q, p}(0, t)}
$$

$$
\begin{aligned}
\leq & C\left(\left\|\mathbf{d}_{0}-\overline{\mathbf{d}}\right\|_{B_{q, p}^{3\left(1-\frac{1}{p}\right)}}+\left\|\mathbf{u}^{n}\right\|_{L_{t}^{\infty}\left(L^{q}\right)}\left\|\nabla\left(\mathbf{d}^{n}-\overline{\mathbf{d}}\right)\right\|_{L_{t}^{p}\left(L^{\infty}\right)}\right. \\
& \left.+\left(\left\|\mathbf{d}^{n}-\overline{\mathbf{d}}\right\|_{L_{t}^{\infty}\left(L^{\infty}\right)}+1\right)\left\|\nabla\left(\mathbf{d}^{n}-\overline{\mathbf{d}}\right)\right\|_{L_{t}^{\infty}\left(L^{q}\right)}\left\|\nabla\left(\mathbf{d}^{n}-\overline{\mathbf{d}}\right)\right\|_{L_{t}^{p}\left(L^{\infty}\right)}\right),
\end{aligned}
$$

and

$$
\begin{aligned}
U^{n+1}(t) \leq & C e^{C t\left(1+\varrho^{n+1}(t)\right)^{\sigma}\left(1+U^{n}(t)\right)^{\frac{1}{\beta}}}\left(U^{0}+\left(t^{\frac{1}{2}-\frac{3}{2 q}}+t^{\frac{1}{3}-\frac{1}{q}}+t^{\frac{2}{3}-\frac{1}{q}}\right)\left(U^{n}(t)\right)^{2}\right. \\
& \left.+t^{\frac{2}{3}-\frac{1}{q}}\left(U^{n}(t)\right)^{3}\right) .
\end{aligned}
$$

Assuming that $t$ is sufficiently small so that

$$
C t^{\frac{3}{2}-\frac{1}{p}-\frac{3}{2 q}} U^{n}(t) \leq \ln 2,
$$


we get from (4.10) that

$$
\varrho^{n+1}(t) \leq 2 \varrho_{0}
$$

and from (4.11) that

$$
\begin{aligned}
U^{n+1}(t) \leq C & e^{2^{\sigma} C t\left(1+U^{n}(t)\right)^{\frac{1}{\beta}}\left(1+\varrho_{0}\right)^{\sigma}}\left(U^{0}+\left(t^{\frac{1}{2}-\frac{3}{2 q}}+t^{\frac{1}{3}-\frac{1}{q}}+t^{\frac{2}{3}-\frac{1}{q}}\right)\left(U^{n}(t)\right)^{2}\right. \\
& \left.+t^{\frac{2}{3}-\frac{1}{q}}\left(U^{n}(t)\right)^{3}\right) .
\end{aligned}
$$

Case 2. $\frac{2}{3}-\frac{1}{q}=\frac{1}{p}$, then $B_{q, p}^{3\left(1-\frac{1}{p}\right)} \hookrightarrow W^{1, \infty-}$ with $\infty$ - denoting any positive number large enough (but not $\infty$ ), using Lemmas 3.1 and 3.3 , we get

$$
\begin{aligned}
& \left\|\mathbf{d}^{n+1}(t)-\overline{\mathbf{d}}\right\|_{B_{q, p}^{3\left(1-\frac{1}{p}\right)}}+\left\|\mathbf{d}^{n+1}-\overline{\mathbf{d}}\right\|_{\mathcal{W}_{q, p}(0, t)} \\
& \leq C\left(\left\|\mathbf{d}_{0}-\overline{\mathbf{d}}\right\|_{B_{q, p}^{3\left(1-\frac{1}{p}\right)}}+t^{\frac{1}{p}}\left\|\mathbf{u}^{n}\right\|_{L_{t}^{\infty}\left(L^{q+}\right)}\left\|\nabla\left(\mathbf{d}^{n}-\overline{\mathbf{d}}\right)\right\|_{L_{t}^{\infty}\left(L^{\infty-}\right)}\right. \\
& \left.\quad+t^{\frac{1}{p}}\left(\left\|\mathbf{d}^{n}-\overline{\mathbf{d}}\right\|_{L_{t}^{\infty}\left(L^{\infty}\right)}+1\right)\left\|\nabla\left(\mathbf{d}^{n}-\overline{\mathbf{d}}\right)\right\|_{L_{t}^{\infty}\left(L^{q+}\right)}\left\|\nabla\left(\mathbf{d}^{n}-\overline{\mathbf{d}}\right)\right\|_{L_{t}^{\infty}\left(L^{\infty}-\right)}\right)
\end{aligned}
$$

and

$$
\begin{array}{rl}
U^{n+1}(t) \leq C & C t\left(1+e^{n+1}(t)\right)^{\sigma}\left(1+U^{n}(t)\right)^{\frac{1}{\beta}}\left(U^{0}+\left(t^{\frac{1}{2}-\frac{3}{2 q}}+t^{\frac{1}{3}-\frac{1}{q}}+t^{\frac{1}{p}}\right)\left(U^{n}(t)\right)^{2}\right. \\
& \left.+t^{\frac{1}{p}}\left(U^{n}(t)\right)^{3}\right) .
\end{array}
$$

Assuming that $t$ is sufficiently small as in (4.12), we get (4.13) and

$$
\begin{aligned}
& U^{n+1}(t) \\
& \leq C e^{2^{\sigma} C t\left(1+U^{n}(t)\right)^{\frac{1}{\beta}}\left(1+\varrho_{0}\right)^{\sigma}}\left(U^{0}+\left(t^{\frac{1}{2}-\frac{3}{2 q}}+t^{\frac{1}{3}-\frac{1}{q}}+t^{\frac{1}{p}}\right)\left(U^{n}(t)\right)^{2}+t^{\frac{1}{p}}\left(U^{n}(t)\right)^{3}\right) .
\end{aligned}
$$

Case 3. $\frac{2}{3}-\frac{1}{q}>\frac{1}{p}>\frac{1}{2}-\frac{3}{2 q}$, then $B_{q, p}^{3\left(1-\frac{1}{p}\right)} \hookrightarrow W^{1, \infty}$, using Lemmas 3.1 and 3.3. we get

$$
\begin{aligned}
& \left\|\mathbf{d}^{n+1}(t)-\overline{\mathbf{d}}\right\|_{B_{q, p}^{3\left(1-\frac{1}{p}\right)}}+\left\|\mathbf{d}^{n+1}-\overline{\mathbf{d}}\right\|_{\mathcal{W}_{q, p}(0, t)} \\
& \leq C\left(\left\|\mathbf{d}_{0}-\overline{\mathbf{d}}\right\|_{B_{q, p}^{3\left(1-\frac{1}{p}\right)}}+t^{\frac{1}{p}}\left\|\mathbf{u}^{n}\right\|_{L_{t}^{\infty}\left(L^{q}\right)}\left\|\nabla\left(\mathbf{d}^{n}-\overline{\mathbf{d}}\right)\right\|_{L_{t}^{\infty}\left(L^{\infty}\right)}\right. \\
& \left.\quad+t^{\frac{1}{p}}\left(\left\|\mathbf{d}^{n}-\overline{\mathbf{d}}\right\|_{L_{t}^{\infty}\left(L^{\infty}\right)}+1\right)\left\|\nabla\left(\mathbf{d}^{n}-\overline{\mathbf{d}}\right)\right\|_{L_{t}^{\infty}\left(L^{q}\right)}\left\|\nabla\left(\mathbf{d}^{n}-\overline{\mathbf{d}}\right)\right\|_{L_{t}^{\infty}\left(L^{\infty}\right)}\right),
\end{aligned}
$$

and again, by choosing $t$ sufficiently small as in (4.12), then (4.13) and (4.14) follow.

Case 4. $\frac{1}{2}-\frac{3}{2 q}=\frac{1}{p}$, then $D_{A_{q}}^{1-\frac{1}{p}, p} \hookrightarrow W^{1, \infty-}$ and $B_{q, p}^{3\left(1-\frac{1}{p}\right)} \hookrightarrow W^{1, \infty}$, using Lemma 3.3. we get

$$
\varrho^{n+1}(t) \leq \varrho_{0} e^{C t^{1-\frac{1}{p}} U^{n}(t)}
$$


and

$$
\begin{aligned}
& \left\|\mathbf{u}^{n+1}(t)\right\|_{D_{A_{q}}^{1-\frac{1}{p}, p}}+\left\|\mathbf{u}^{n+1}\right\|_{L_{t}^{p}\left(W^{2, q}\right)}+\left\|\partial_{t} \mathbf{u}^{n+1}\right\|_{L_{t}^{p}\left(L^{q}\right)}+\left\|P^{n+1}\right\|_{L_{t}^{p}\left(W^{1, q}\right)} \\
& \leq C e^{C t\left(1+\left\|\rho^{n+1}\right\|_{L_{t}^{\infty}\left(W^{1, r}\right)}\right)^{\sigma}\left(1+\left\|\mathbf{u}^{n}\right\|_{L_{t}^{\infty}\left(L^{q}\right)}\right)^{\frac{1}{\beta}}} \\
& \quad \times\left(\left\|\mathbf{u}_{0}\right\|_{D_{A_{A_{q}}^{1-\frac{1}{p}, p}}+t^{\frac{1}{p}}\left\|\mathbf{u}^{n}\right\|_{L_{t}^{\infty}\left(L^{q+}\right)}\left\|\nabla \mathbf{u}^{n}\right\|_{L_{t}^{\infty}\left(L^{\infty-}\right)}} \quad+\left\|\nabla\left(\mathbf{d}^{n}-\overline{\mathbf{d}}\right)\right\|_{L_{t}^{\infty}\left(L^{q}\right)}\left\|\triangle\left(\mathbf{d}^{n}-\overline{\mathbf{d}}\right)\right\|_{L_{t}^{p}\left(L^{\infty}\right)}\right)
\end{aligned}
$$

$U^{n+1}(t) \leq C e^{C t\left(1+\varrho^{n+1}(t)\right)^{\sigma}\left(1+U^{n}(t)\right)^{\frac{1}{\beta}}}\left(U^{0}+\left(t^{\frac{1}{3}-\frac{1}{q}}+2 t^{\frac{1}{p}}\right)\left(U^{n}(t)\right)^{2}+t^{\frac{1}{p}}\left(U^{n}(t)\right)^{3}\right)$.

Assuming that $t$ is sufficiently small so that

$$
C t^{1-\frac{1}{p}} U^{n}(t) \leq \ln 2
$$

we get from (4.15) that (4.13) holds and

$$
U^{n+1}(t) \leq C e^{2^{\sigma} C t\left(1+U^{n}(t)\right)^{\frac{1}{\beta}}\left(1+\varrho_{0}\right)^{\sigma}}\left(U^{0}+\left(t^{\frac{1}{3}-\frac{1}{q}}+2 t^{\frac{1}{p}}\right)\left(U^{n}(t)\right)^{2}+t^{\frac{1}{p}}\left(U^{n}(t)\right)^{3}\right) .
$$

Case 5. $\frac{1}{2}-\frac{3}{2 q}>\frac{1}{p}>\frac{1}{3}-\frac{1}{q}$, then $D_{A_{q}}^{1-\frac{1}{p}, p} \hookrightarrow W^{1, \infty}$ and $B_{q, p}^{3\left(1-\frac{1}{p}\right)} \hookrightarrow W^{1, \infty}$, using Lemma 3.3. we get

$$
\begin{aligned}
& \left\|\mathbf{u}^{n+1}(t)\right\|_{D_{A_{q}}^{1-\frac{1}{p}, p}}+\left\|\mathbf{u}^{n+1}\right\|_{L_{t}^{p}\left(W^{2, q}\right)}+\left\|\partial_{t} \mathbf{u}^{n+1}\right\|_{L_{t}^{p}\left(L^{q}\right)}+\left\|P^{n+1}\right\|_{L_{t}^{p}\left(W^{1, q}\right)} \\
& \leq C e^{C t\left(1+\left\|\rho^{n+1}\right\|_{L_{t}^{\infty}\left(W^{1, r}\right)}\right)^{\sigma}\left(1+\left\|\mathbf{u}^{n}\right\|_{L_{t}^{\infty}\left(L^{q}\right)}\right)^{\frac{1}{\beta}}} \\
& \quad \times\left(\left\|\mathbf{u}_{0}\right\|_{D_{A_{q}}^{1-\frac{1}{p}, p}}+t^{\frac{1}{p}}\left\|\mathbf{u}^{n}\right\|_{L_{t}^{\infty}\left(L^{q}\right)}\left\|\nabla \mathbf{u}^{n}\right\|_{L_{t}^{\infty}\left(L^{\infty}\right)}\right. \\
& \left.\quad+\left\|\nabla\left(\mathbf{d}^{n}-\overline{\mathbf{d}}\right)\right\|_{L_{t}^{\infty}\left(L^{q}\right)}\left\|\triangle\left(\mathbf{d}^{n}-\overline{\mathbf{d}}\right)\right\|_{L_{t}^{p}\left(L^{\infty}\right)}\right),
\end{aligned}
$$

and (4.16) follows. Moreover, by choosing $t$ sufficiently small as in (4.17), then (4.13) and (4.18) follow.

Case 6. $\frac{1}{3}-\frac{1}{q}=\frac{1}{p}$, then $D_{A_{q}}^{1-\frac{1}{p}, p} \hookrightarrow W^{1, \infty}$ and $B_{q, p}^{3\left(1-\frac{1}{p}\right)} \hookrightarrow W^{2, \infty-}$, we get

$$
\begin{aligned}
& \left\|\mathbf{u}^{n+1}(t)\right\|_{D_{A_{q}}^{1-\frac{1}{p}, p}}+\left\|\mathbf{u}^{n+1}\right\|_{L_{t}^{p}\left(W^{2, q}\right)}+\left\|\partial_{t} \mathbf{u}^{n+1}\right\|_{L_{t}^{p}\left(L^{q}\right)}+\left\|P^{n+1}\right\|_{L_{t}^{p}\left(W^{1, q}\right)} \\
& \leq C e^{C t\left(1+\left\|\rho^{n+1}\right\|_{L_{t}^{\infty}\left(W^{1, r}\right)}\right)^{\sigma}\left(1+\left\|\mathbf{u}^{n}\right\|_{L_{t}^{\infty}\left(L^{q}\right)}\right)^{\frac{1}{\beta}}} \\
& \quad \times\left(\left\|\mathbf{u}_{0}\right\|_{D_{A_{q}}^{1-\frac{1}{p}, p}}+t^{\frac{1}{p}}\left\|\mathbf{u}^{n}\right\|_{L_{t}^{\infty}\left(L^{q}\right)}\left\|\nabla \mathbf{u}^{n}\right\|_{L_{t}^{\infty}\left(L^{\infty}\right)}\right. \\
& \left.\quad+t^{\frac{1}{p}}\left\|\nabla\left(\mathbf{d}^{n}-\overline{\mathbf{d}}\right)\right\|_{L_{t}^{\infty}\left(L^{q+}\right)}\left\|\triangle\left(\mathbf{d}^{n}-\overline{\mathbf{d}}\right)\right\|_{L_{t}^{p}\left(L^{\infty-}\right)}\right)
\end{aligned}
$$

and

$$
U^{n+1}(t) \leq C e^{C t\left(1+\varrho^{n+1}(t)\right)^{\sigma}\left(1+U^{n}(t)\right)^{\frac{1}{\beta}}}\left(U^{0}+3 t^{\frac{1}{p}}\left(U^{n}(t)\right)^{2}+t^{\frac{1}{p}}\left(U^{n}(t)\right)^{3}\right) .
$$


Assuming that $t$ is sufficiently small as in (4.17), we get (4.13) and

$$
U^{n+1}(t) \leq C e^{2^{\sigma} C t\left(1+U^{n}(t)\right)^{\frac{1}{\beta}}\left(1+\varrho_{0}\right)^{\sigma}}\left(U^{0}+3 t^{\frac{1}{p}}\left(U^{n}(t)\right)^{2}+t^{\frac{1}{p}}\left(U^{n}(t)\right)^{3}\right) .
$$

Case 7. $\frac{1}{3}-\frac{1}{q}>\frac{1}{p}$, then $D_{A_{q}}^{1-\frac{1}{p}, p} \hookrightarrow W^{1, \infty}$ and $B_{q, p}^{3\left(1-\frac{1}{p}\right)} \hookrightarrow W^{2, \infty}$, we get

$$
\begin{aligned}
& \left\|\mathbf{u}^{n+1}(t)\right\|_{D_{A_{q}}^{1-\frac{1}{p}, p}}+\left\|\mathbf{u}^{n+1}\right\|_{L_{t}^{p}\left(W^{2, q}\right)}+\left\|\partial_{t} \mathbf{u}^{n+1}\right\|_{L_{t}^{p}\left(L^{q}\right)}+\left\|P^{n+1}\right\|_{L_{t}^{p}\left(W^{1, q}\right)} \\
& \leq C e^{C t\left(1+\left\|\rho^{n+1}\right\|_{L_{t}^{\infty}\left(W^{1, r}\right)}\right)^{\sigma}\left(1+\left\|\mathbf{u}^{n}\right\|_{L_{t}^{\infty}\left(L^{q}\right)}\right)^{\frac{1}{\beta}}} \\
& \quad \times\left(\left\|\mathbf{u}_{0}\right\|_{D_{A_{q}}^{1-\frac{1}{p}, p}}+t^{\frac{1}{p}}\left\|\mathbf{u}^{n}\right\|_{L_{t}^{\infty}\left(L^{q}\right)}\left\|\nabla \mathbf{u}^{n}\right\|_{L_{t}^{\infty}\left(L^{\infty}\right)}\right. \\
& \left.\quad+t^{\frac{1}{p}}\left\|\nabla\left(\mathbf{d}^{n}-\overline{\mathbf{d}}\right)\right\|_{L_{t}^{\infty}\left(L^{q}\right)}\left\|\triangle\left(\mathbf{d}^{n}-\overline{\mathbf{d}}\right)\right\|_{L_{t}^{p}\left(L^{\infty}\right)}\right),
\end{aligned}
$$

and (4.19) follows. Assuming that $t$ is sufficiently small as in (4.17), then (4.13) and (4.20) hold.

Hence, for Cases 1, 2, and 3, if we assume that $U^{n}(t) \leq 4 C U^{0}$ on $\left[0, T_{*}\right]$ with

$$
\begin{aligned}
T_{*}=\min \left\{\left(\frac{\ln 2}{4 C^{2} U^{0}}\right)^{\frac{3 p q-2 q-3 p}{2 p q}}, \frac{\ln 2}{2^{\sigma} C\left(1+\varrho_{0}\right)^{\sigma}\left(1+4 C U^{0}\right)^{\frac{1}{\beta}}},\right. \\
\left.\left(\frac{1}{16 C^{2} U^{0}\left(3+4 C U^{0}\right)}\right)^{\frac{3 q}{q-3}}\right\} \leq 1
\end{aligned}
$$

or

$$
\begin{aligned}
1<T_{*}=\min \left\{\left(\frac{\ln 2}{4 C^{2} U^{0}}\right)^{\frac{3 p q-2 q-3 p}{2 p q}}, \frac{\ln 2}{2^{\sigma} C\left(1+\varrho_{0}\right)^{\sigma}\left(1+4 C U^{0}\right)^{\frac{1}{\beta}}},\right. \\
\left.\left(\frac{1}{16 C^{2} U^{0}\left(3+4 C U^{0}\right)}\right)^{\max \left\{p, \frac{3 q}{2 q-3}\right\}}\right\},
\end{aligned}
$$

and for Cases $4,5,6$, and 7 , if we assume that $U^{n}(t) \leq 4 C U^{0}$ on $\left[0, T_{*}\right]$ with

$$
\begin{array}{r}
T_{*}=\min \left\{\left(\frac{\ln 2}{4 C^{2} U^{0}}\right)^{\frac{p}{p-1}}, \frac{\ln 2}{2^{\sigma} C\left(1+\varrho_{0}\right)^{\sigma}\left(1+4 C U^{0}\right)^{\frac{1}{\beta}}},\right. \\
\left.\left(\frac{1}{16 C^{2} U^{0}\left(3+4 C U^{0}\right)}\right)^{\max \left\{p, \frac{3 q}{q-3}\right\}}\right\} \leq 1
\end{array}
$$

or

$$
\begin{gathered}
1<T_{*}=\min \left\{\left(\frac{\ln 2}{4 C^{2} U^{0}}\right)^{\frac{p}{p-1}}, \frac{\ln 2}{2^{\sigma} C\left(1+\varrho_{0}\right)^{\sigma}\left(1+4 C U^{0}\right)^{\frac{1}{\beta}}},\right. \\
\left.\left(\frac{1}{16 C^{2} U^{0}\left(3+4 C U^{0}\right)}\right)^{p}\right\}
\end{gathered}
$$

then a direct computation yields

$$
U^{n+1}(t) \leq 4 C U^{0} \text { on }\left[0, T_{*}\right]
$$


Coming back to (4.10), we conclude that the sequence $\left\{\left(\rho^{n}, \mathbf{u}^{n}, P^{n}, \mathbf{d}^{n}-\overline{\mathbf{d}}\right)\right\}$ is uniformly bounded in $M_{T_{*}}^{p, q, r}$. More precisely, we have proved the following estimates:

Lemma 4.1. For all $t \in\left[0, T_{*}\right]$ with $T_{*}$ satisfying (4.21) or (4.22) for Cases 1-3, and (4.23) or (4.24) for Cases 4-7,

$$
\varrho^{n}(t) \leq 2 \varrho_{0} \text { and } U^{n}(t) \leq 4 C U^{0} .
$$

\subsection{Convergence of the approximate sequence.}

Lemma 4.2. There exists $T_{0}$ such that $\left\{\left(\rho^{n}, \mathbf{u}^{n}, P^{n}, \mathbf{d}^{n}-\overline{\mathbf{d}}\right)\right\}_{n=1}^{\infty}$ is a Cauchy sequence in $M_{T_{0}}^{p, s, r}$ and thus converges.

Proof. Let

$$
\bar{\rho}^{n}:=\rho^{n+1}-\rho^{n}, \quad \overline{\mathbf{u}}^{n}:=\mathbf{u}^{n+1}-\mathbf{u}^{n}, \quad \bar{P}^{n}:=P^{n+1}-P^{n}, \quad \overline{\mathbf{d}}^{n}:=\mathbf{d}^{n+1}-\mathbf{d}^{n} .
$$

Define

$$
\begin{aligned}
\bar{U}^{n}(t):= & \left\|\overline{\mathbf{u}}^{n}\right\|_{L_{t}^{\infty}\left(D_{A_{s}}^{1-\frac{1}{p}, p}\right)}+\left\|\overline{\mathbf{u}}^{n}\right\|_{L_{t}^{p}\left(W^{2, s}\right)}+\left\|\partial_{t} \overline{\mathbf{u}}^{n}\right\|_{L_{t}^{p}\left(L^{s}\right)} \\
& +\left\|\nabla \bar{P}^{n}\right\|_{L_{t}^{p}\left(L^{s}\right)}+\left\|\overline{\mathbf{d}}^{n}\right\|_{L_{t}^{\infty}\left(B_{s, p}^{3\left(1-\frac{1}{p}\right)}\right)}+\left\|\overline{\mathbf{d}}^{n}\right\|_{\mathcal{W}_{s, p}(0, t)} .
\end{aligned}
$$

It is easy to verify that $\left(\bar{\rho}^{n}, \overline{\mathbf{u}}^{n}, \bar{P}^{n}, \overline{\mathbf{d}}^{n}\right)$ satisfies

$$
\left\{\begin{array}{c}
\partial_{t} \bar{\rho}^{n}+\mathbf{u}^{n} \cdot \nabla \bar{\rho}^{n}=-\overline{\mathbf{u}}^{n-1} \cdot \nabla \rho^{n} \\
\rho^{n+1} \partial_{t} \overline{\mathbf{u}}^{n}-\triangle \overline{\mathbf{u}}^{n}+\nabla \bar{P}^{n} \\
=-\bar{\rho}^{n}\left(\partial_{t} \mathbf{u}^{n}+\mathbf{u}^{n} \cdot \nabla \mathbf{u}^{n}\right)-\rho^{n}\left(\mathbf{u}^{n} \cdot \nabla \overline{\mathbf{u}}^{n-1}+\overline{\mathbf{u}}^{n-1} \cdot \nabla \mathbf{u}^{n-1}\right) \\
\quad-\nabla \cdot\left(\left(\nabla \overline{\mathbf{d}}^{n-1}\right)^{\top} \nabla\left(\mathbf{d}^{n}-\overline{\mathbf{d}}\right)\right)-\nabla \cdot\left(\left(\nabla\left(\mathbf{d}^{n-1}-\overline{\mathbf{d}}\right)\right)^{\top} \nabla \overline{\mathbf{d}}^{n-1}\right), \\
\quad+\left(\left(\nabla\left(\mathbf{d}^{n}-\overline{\mathbf{d}}\right)+\nabla\left(\mathbf{d}^{n-1}-\overline{\mathbf{d}}\right)\right): \nabla \overline{\mathbf{d}}^{n-1}\right)\left(\mathbf{d}^{n-1}-\overline{\mathbf{d}}\right) \\
\quad+\left(\left(\nabla\left(\mathbf{d}^{n}-\overline{\mathbf{d}}\right)+\nabla\left(\mathbf{d}^{n-1}-\overline{\mathbf{d}}\right)\right): \nabla \overline{\mathbf{d}}^{n-1}\right) \overline{\mathbf{d}} \\
\quad \overline{\mathbf{d}}^{n}=-\overline{\mathbf{u}}^{n-1} \cdot \nabla\left(\mathbf{d}^{n}-\overline{\mathbf{d}}\right)-\mathbf{u}^{n-1} \cdot \nabla \overline{\mathbf{d}}^{n-1}+\mid \nabla\left(\mathbf{d}^{n}-\overline{\mathbf{d}}\right) \overline{\mathbf{d}}^{n-1} \\
\quad{ }_{\Omega} \bar{P}^{n} d \mathbf{x}=0
\end{array}\right.
$$

with the initial-boundary conditions:

$$
\begin{aligned}
\left.\left(\bar{\rho}^{n}, \overline{\mathbf{u}}^{n}, \overline{\mathbf{d}}^{n}\right)\right|_{t=0} & =(0,0,0), \\
\left.\left(\overline{\mathbf{u}}^{n}, \partial_{\nu} \overline{\mathbf{d}}^{n}\right)\right|_{\partial \Omega} & =(0,0) .
\end{aligned}
$$

Applying Theorem 3.1 to

$$
\left\{\begin{aligned}
\partial_{t} \overline{\mathbf{d}}^{n}-\triangle \overline{\mathbf{d}}^{n}= & -\overline{\mathbf{u}}^{n-1} \cdot \nabla\left(\mathbf{d}^{n}-\overline{\mathbf{d}}\right)-\mathbf{u}^{n-1} \cdot \nabla \overline{\mathbf{d}}^{n-1}+\left|\nabla\left(\mathbf{d}^{n}-\overline{\mathbf{d}}\right)\right|^{2} \overline{\mathbf{d}}^{n-1} \\
& +\left(\left(\nabla\left(\mathbf{d}^{n}-\overline{\mathbf{d}}\right)+\nabla\left(\mathbf{d}^{n-1}-\overline{\mathbf{d}}\right)\right): \nabla \overline{\mathbf{d}}^{n-1}\right)\left(\mathbf{d}^{n-1}-\overline{\mathbf{d}}\right) \\
& +\left(\left(\nabla\left(\mathbf{d}^{n}-\overline{\mathbf{d}}\right)+\nabla\left(\mathbf{d}^{n-1}-\overline{\mathbf{d}}\right)\right): \nabla \overline{\mathbf{d}}^{n-1}\right) \overline{\mathbf{d}} \\
\left.\overline{\mathbf{d}}^{n}\right|_{t=0}=0,\left.\quad \partial_{\nu} \overline{\mathbf{d}}^{n}\right|_{\partial \Omega}=0 &
\end{aligned}\right.
$$

and applying Theorem 3.2 to

$$
\left\{\begin{array}{l}
\rho^{n+1} \partial_{t} \overline{\mathbf{u}}^{n}-\triangle \overline{\mathbf{u}}^{n}+\nabla \bar{P}^{n} \\
\quad=-\bar{\rho}^{n}\left(\partial_{t} \mathbf{u}^{n}+\mathbf{u}^{n} \cdot \nabla \mathbf{u}^{n}\right)-\rho^{n}\left(\mathbf{u}^{n} \cdot \nabla \overline{\mathbf{u}}^{n-1}+\overline{\mathbf{u}}^{n-1} \cdot \nabla \mathbf{u}^{n-1}\right) \\
\quad-\nabla \cdot\left(\left(\nabla \overline{\mathbf{d}}^{n-1}\right)^{\top} \nabla\left(\mathbf{d}^{n}-\overline{\mathbf{d}}\right)\right)-\nabla \cdot\left(\left(\nabla\left(\mathbf{d}^{n-1}-\overline{\mathbf{d}}\right)\right)^{\top} \nabla \overline{\mathbf{d}}^{n-1}\right), \\
\nabla \cdot \overline{\mathbf{u}}^{n}=0, \quad \int_{\Omega} \bar{P}^{n} d \mathbf{x}=0, \\
\left.\overline{\mathbf{u}}^{n}\right|_{t=0}=0,\left.\quad \overline{\mathbf{u}}^{n}\right|_{\partial \Omega}=0,
\end{array}\right.
$$


we have

$$
\begin{aligned}
& \bar{U}^{n}(t) \leq C\left(\left\|\bar{\rho}^{n}\left(\partial_{t} \mathbf{u}^{n}+\mathbf{u}^{n} \cdot \nabla \mathbf{u}^{n}\right)\right\|_{L_{t}^{p}\left(L^{s}\right)}+\left\|\mathbf{u}^{n} \cdot \nabla \overline{\mathbf{u}}^{n-1}+\overline{\mathbf{u}}^{n-1} \cdot \nabla \mathbf{u}^{n-1}\right\|_{L_{t}^{p}\left(L^{s}\right)}\right. \\
& +\left\|\nabla \cdot\left(\left(\nabla \overline{\mathbf{d}}^{n-1}\right)^{\top} \nabla\left(\mathbf{d}^{n}-\overline{\mathbf{d}}\right)\right)+\nabla \cdot\left(\left(\nabla\left(\mathbf{d}^{n-1}-\overline{\mathbf{d}}\right)\right)^{\top} \nabla \overline{\mathbf{d}}^{n-1}\right)\right\|_{L_{t}^{p}\left(L^{s}\right)} \\
& +\left\|\overline{\mathbf{u}}^{n-1} \cdot \nabla\left(\mathbf{d}^{n}-\overline{\mathbf{d}}\right)+\mathbf{u}^{n-1} \cdot \nabla \overline{\mathbf{d}}^{n-1}\right\|_{L_{t}^{p}\left(L^{s}\right)}+\left\|\left|\nabla\left(\mathbf{d}^{n}-\overline{\mathbf{d}}\right)\right|^{2} \overline{\mathbf{d}}^{n-1}\right\|_{L_{t}^{p}\left(L^{s}\right)} \\
& +\|\left(\left(\nabla\left(\mathbf{d}^{n}-\overline{\mathbf{d}}\right)+\nabla\left(\mathbf{d}^{n-1}-\overline{\mathbf{d}}\right)\right): \nabla \overline{\mathbf{d}}^{n-1}\right)\left(\mathbf{d}^{n-1}-\overline{\mathbf{d}}\right) \\
& \left.+\left(\left(\nabla\left(\mathbf{d}^{n}-\overline{\mathbf{d}}\right)+\nabla\left(\mathbf{d}^{n-1}-\overline{\mathbf{d}}\right)\right): \nabla \overline{\mathbf{d}}^{n-1}\right) \overline{\mathbf{d}} \|_{L_{t}^{p}\left(L^{s}\right)}\right) \\
& \leq C\left(\left\|\bar{\rho}^{n}\right\|_{L_{t}^{\infty}\left(L^{r}\right)}\left(\left\|\partial_{t} \mathbf{u}^{n}\right\|_{L_{t}^{p}\left(L^{q}\right)}+\left\|\mathbf{u}^{n}\right\|_{L_{t}^{\infty}\left(L^{q}\right)}\left\|\nabla \mathbf{u}^{n}\right\|_{L_{t}^{p}\left(L^{\infty}\right)}\right)\right. \\
& +\left\|\mathbf{u}^{n}\right\|_{L_{t}^{\infty}\left(L^{q}\right)}\left\|\nabla \overline{\mathbf{u}}^{n-1}\right\|_{L_{t}^{p}\left(L^{r}\right)}+\left\|\overline{\mathbf{u}}^{n-1}\right\|_{L_{t}^{\infty}\left(L^{s}\right)}\left\|\nabla \mathbf{u}^{n-1}\right\|_{L_{t}^{p}\left(L^{\infty}\right)} \\
& +\left\|\nabla\left(\mathbf{d}^{n}-\overline{\mathbf{d}}\right)\right\|_{L_{t}^{\infty}\left(L^{q}\right)}\left\|\triangle \overline{\mathbf{d}}^{n-1}\right\|_{L_{t}^{p}\left(L^{r}\right)}+\left\|\nabla \overline{\mathbf{d}}^{n-1}\right\|_{L_{t}^{\infty}\left(L^{s}\right)}\left\|\triangle\left(\mathbf{d}^{n}-\overline{\mathbf{d}}\right)\right\|_{L_{t}^{p}\left(L^{\infty}\right)} \\
& +\left\|\nabla \overline{\mathbf{d}}^{n-1}\right\|_{L_{t}^{\infty}\left(L^{s}\right)}\left\|\triangle\left(\mathbf{d}^{n}-\overline{\mathbf{d}}\right)\right\|_{L_{t}^{p}\left(L^{\infty}\right)}+\left\|\nabla\left(\mathbf{d}^{n-1}-\overline{\mathbf{d}}\right)\right\|_{L_{t}^{\infty}\left(L^{q}\right)}\left\|\triangle \overline{\mathbf{d}}^{n-1}\right\|_{L_{t}^{p}\left(L^{r}\right)} \\
& +\left\|\overline{\mathbf{u}}^{n-1}\right\|_{L_{t}^{\infty}\left(L^{s}\right)}\left\|\nabla\left(\mathbf{d}^{n}-\overline{\mathbf{d}}\right)\right\|_{L_{t}^{p}\left(L^{\infty}\right)}+\left\|\mathbf{u}^{n-1}\right\|_{L_{t}^{\infty}\left(L^{q}\right)}\left\|\nabla \overline{\mathbf{d}}^{n-1}\right\|_{L_{t}^{p}\left(L^{r}\right)} \\
& +\left\|\nabla\left(\mathbf{d}^{n}-\overline{\mathbf{d}}\right)\right\|_{L_{t}^{\infty}\left(L^{q}\right)}\left\|\nabla\left(\mathbf{d}^{n}-\overline{\mathbf{d}}\right)\right\|_{L_{t}^{p}\left(L^{r}\right)}\left\|\overline{\mathbf{d}}^{n-1}\right\|_{L_{t}^{\infty}\left(L^{\infty}\right)} \\
& +\left\|\nabla \overline{\mathbf{d}}^{n-1}\right\|_{L_{t}^{\infty}\left(L^{s}\right)}\left(\left\|\nabla\left(\mathbf{d}^{n}-\overline{\mathbf{d}}\right)\right\|_{L_{t}^{p}\left(L^{\infty}\right)}+\left\|\nabla\left(\mathbf{d}^{n-1}-\overline{\mathbf{d}}\right)\right\|_{L_{t}^{p}\left(L^{\infty}\right)}\right) \\
& \left.\left(\left\|\mathbf{d}^{n-1}-\overline{\mathbf{d}}\right\|_{L_{t}^{\infty}\left(L^{\infty}\right)}+1\right)\right) \text {. }
\end{aligned}
$$

Note that if $\frac{1}{2}-\frac{3}{2 q}<\frac{1}{p}$, using Young's inequality, Lemma 3.1 yields

$$
\left\|\nabla \overline{\mathbf{u}}^{n-1}\right\|_{L_{t}^{p}\left(L^{r}\right)} \leq C t^{\frac{1}{2}-\frac{3}{2 q}}\left(\left\|\overline{\mathbf{u}}^{n-1}\right\|_{L_{t}^{\infty}\left(D_{A_{s}}^{1-\frac{1}{p}, p}\right)}^{1-\theta}+\left\|\overline{\mathbf{u}}^{n-1}\right\|_{L_{t}^{p}\left(W^{2, s}\right)}^{\theta}\right) .
$$

If $\frac{1}{2}-\frac{3}{2 q}>\frac{1}{p}$, we have $D_{A_{s}}^{1-\frac{1}{p}, p} \hookrightarrow W^{1, r}$ so that the above inequality holds with the power of $t$ replaced by $t^{\frac{1}{p}}$. As for $\frac{1}{2}-\frac{3}{2 q}=\frac{1}{p}$, since

$$
\left\|\mathbf{u}^{n} \cdot \nabla \overline{\mathbf{u}}^{n-1}\right\|_{L_{t}^{p}\left(L^{s}\right)} \leq\left\|\mathbf{u}^{n}\right\|_{L_{t}^{\infty}\left(L^{q+}\right)}\left\|\nabla \overline{\mathbf{u}}^{n-1}\right\|_{L_{t}^{p}\left(L^{r-}\right)}
$$

and $D_{A_{s}}^{1-\frac{1}{p}, p} \hookrightarrow L^{q+}, D_{A_{s}}^{1-\frac{1}{p}, p} \hookrightarrow W^{1, r-}$ with $q+($ resp. $r-$ ) slightly greater (resp. smaller) than $q$ (resp. $r$ ), we still have

$$
\left\|\mathbf{u}^{n} \cdot \nabla \overline{\mathbf{u}}^{n-1}\right\|_{L_{t}^{p}\left(L^{s}\right)} \leq t^{\frac{1}{p}}\left\|\mathbf{u}^{n}\right\|_{L_{t}^{\infty}\left(D_{A_{s}}^{1-\frac{1}{p}, p}\right)}\left\|\overline{\mathbf{u}}^{n-1}\right\|_{L_{t}^{\infty}\left(D_{A_{s}}^{1-\frac{1}{p}, p}\right)}
$$

The other terms such as $\left\|\triangle \overline{\mathbf{d}}^{n-1}\right\|_{L_{t}^{p}\left(L^{r}\right)},\left\|\nabla \overline{\mathbf{d}}^{n-1}\right\|_{L_{t}^{p}\left(L^{r}\right)}$ and $\left\|\triangle\left(\mathbf{d}^{n}-\overline{\mathbf{d}}\right)\right\|_{L_{t}^{p}\left(L^{r}\right)}$ may be handled via a similar technique by using the Besov space $B_{s, p}^{3\left(1-\frac{1}{p}\right)}$.

To simplify the presentation, assume from now on that $\frac{2}{3}-\frac{1}{q}<\frac{1}{p}$ so that Lemmas 3.13 .3 can be applied. Otherwise, according to the arguments above, we would get $t^{\frac{1}{p}}$ instead of $t^{\frac{2}{3}-\frac{1}{q}}, t^{\frac{1}{2}-\frac{3}{2 q}}$, or $t^{\frac{1}{3}-\frac{1}{q}}$ below once their exponents are greater than or equal to $\frac{1}{p}$. 
Hence, for all $t \in\left[0, T_{*}\right]$, taking advantage of (4.25), the embedding $B_{s, p}^{3\left(1-\frac{1}{p}\right)} \hookrightarrow$ $W^{1, s}$ and Lemmas 3.1]3.3, we get

$$
\begin{aligned}
& \bar{U}^{n}(t) \leq C\left(\left\|\bar{\rho}^{n}\right\|_{L_{t}^{\infty}\left(L^{r}\right)}+\left\|\nabla \overline{\mathbf{u}}^{n-1}\right\|_{L_{t}^{p}\left(L^{r}\right)}+t^{\frac{1}{2}-\frac{3}{2 q}}\left\|\overline{\mathbf{u}}^{n-1}\right\|_{L_{t}^{\infty}\left(L^{s}\right)}+\left\|\triangle \overline{\mathbf{d}}^{n-1}\right\|_{L_{t}^{p}\left(L^{r}\right)}\right. \\
& +t^{\frac{1}{3}-\frac{1}{q}}\left\|\nabla \overline{\mathbf{d}}^{n-1}\right\|_{L_{t}^{\infty}\left(L^{s}\right)}+t^{\frac{2}{3}-\frac{1}{q}}\left\|\overline{\mathbf{u}}^{n-1}\right\|_{L_{t}^{\infty}\left(L^{s}\right)}+\left\|\nabla \overline{\mathbf{d}}^{n-1}\right\|_{L_{t}^{p}\left(L^{r}\right)} \\
& \left.+t^{\frac{2}{3}-\frac{1}{q}}\left\|\overline{\mathbf{d}}^{n-1}\right\|_{L_{t}^{\infty}\left(L^{\infty}\right)}+t^{\frac{2}{3}-\frac{1}{q}}\left\|\nabla \overline{\mathbf{d}}^{n-1}\right\|_{L_{t}^{\infty}\left(L^{s}\right)}\right) \\
& \leq C\left(\left\|\bar{\rho}^{n}\right\|_{L_{t}^{\infty}\left(L^{r}\right)}+\left(t^{\frac{1}{2}-\frac{3}{2 q}}+t^{\frac{1}{3}-\frac{1}{q}}+t^{\frac{2}{3}-\frac{1}{q}}\right) \bar{U}^{n-1}(t)\right) \text {. }
\end{aligned}
$$

Moreover, multiplying

$$
\partial_{t} \bar{\rho}^{n}+\mathbf{u}^{n} \cdot \nabla \bar{\rho}^{n}=-\overline{\mathbf{u}}^{n-1} \cdot \nabla \rho^{n}
$$

by $\left|\bar{\rho}^{n}\right|^{r-2} \bar{\rho}^{n}$ and integrating over $\Omega$, using $\nabla \cdot \mathbf{u}^{n}=0$ and the zero boundary condition, by Hölder's inequality we have

$$
\begin{aligned}
\frac{1}{r} \frac{d}{d t}\left\|\bar{\rho}^{n}\right\|_{L^{r}}^{r} & =-\frac{1}{r} \int_{\Omega} \mathbf{u}^{n} \cdot \nabla\left(\left|\bar{\rho}^{n}\right|^{r}\right) d \mathbf{x}-\int_{\Omega}\left|\bar{\rho}^{n}\right|^{r-2} \bar{\rho}^{n} \overline{\mathbf{u}}^{n-1} \cdot \nabla \bar{\rho}^{n} d \mathbf{x} \\
& \leq\left\|\bar{\rho}^{n}\right\|_{L^{r}}^{r-1}\left\|\overline{\mathbf{u}}^{n-1} \cdot \nabla \bar{\rho}^{n}\right\|_{L^{r}} .
\end{aligned}
$$

By Hölder's inequality, (4.25) and the embedding

$$
W^{2, s}(\Omega) \hookrightarrow L^{\infty}(\Omega) \quad \text { as } \quad s=\frac{q r}{q+r}>\frac{3}{2},
$$

we eventually obtain

$$
\begin{aligned}
\left\|\bar{\rho}^{n}(t)\right\|_{L^{r}} & \leq \int_{0}^{t}\left\|\overline{\mathbf{u}}^{n-1}(\tau) \cdot \nabla \rho^{n}(\tau)\right\|_{L^{r}} d \tau \\
& \leq t^{1-\frac{1}{p}}\left\|\overline{\mathbf{u}}^{n-1}\right\|_{L_{t}^{p}\left(L^{\infty}\right)}\left\|\nabla \rho^{n}\right\|_{L_{t}^{\infty}\left(L^{r}\right)} \\
& \leq C t^{1-\frac{1}{p}} \bar{U}^{n-1}(t) .
\end{aligned}
$$

Inserting (4.27) into (4.26), we get for $t \in\left[0, T_{*}\right]$,

$$
\bar{U}^{n}(t) \leq C\left(t^{1-\frac{1}{p}}+t^{\frac{1}{2}-\frac{3}{2 q}}+t^{\frac{1}{3}-\frac{1}{q}}+t^{\frac{2}{3}-\frac{1}{q}}\right) \bar{U}^{n-1}(t) .
$$

If we choose $T_{0} \in\left(0, T_{*}\right]$ such that

$$
C\left(T_{0}^{1-\frac{1}{p}}+T_{0}^{\frac{1}{2}-\frac{3}{2 q}}+T_{0}^{\frac{1}{3}-\frac{1}{q}}+T_{0}^{\frac{2}{3}-\frac{1}{q}}\right) \leq \frac{1}{2},
$$

then $\left\{\left(\rho^{n}, \mathbf{u}^{n}, P^{n}, \mathbf{d}^{n}-\overline{\mathbf{d}}\right)\right\}$ is a Cauchy sequence in $M_{T_{0}}^{p, s, r}$ and thus converges in $M_{T_{0}}^{p, s, r}$.

We remark here that the time of existence $T_{0}$ depends (continuously) on the norms of the data, on the bound for the density, on the domain and on the regularity parameters. 
4.4. The limit is a solution. Let $(\rho, \mathbf{u}, P, \mathbf{d}-\overline{\mathbf{d}}) \in M_{T_{0}}^{p, s, r}$ be the limit of the sequence $\left\{\left(\rho^{n}, \mathbf{u}^{n}, P^{n}, \mathbf{d}^{n}-\overline{\mathbf{d}}\right)\right\}_{n=1}^{\infty}$ in $M_{T_{0}}^{p, s, r}$. Passing to the limit in (4.5) and (4.25) yields

$$
\begin{gathered}
0<\check{\rho} \leq \rho(\mathbf{x}, t) \leq \hat{\rho}, \quad(\mathbf{x}, t) \in \Omega \times\left[0, T_{0}\right] \quad \text { and } \quad \rho \in L^{\infty}\left(0, T_{0} ; W^{1, r}(\Omega)\right), \\
\mathbf{u} \in L^{\infty}\left(0, T_{0} ; D_{A_{q}}^{1-\frac{1}{p}, p}\right) \cap L^{p}\left(0, T_{0} ; W^{2, q}(\Omega)\right), \quad \partial_{t} \mathbf{u} \in L^{p}\left(0, T_{0} ; L^{q}(\Omega)\right), \\
\mathbf{d}-\overline{\mathbf{d}} \in L^{\infty}\left(0, T_{0} ; B_{q, p}^{3\left(1-\frac{1}{p}\right)}\right) \cap L^{p}\left(0, T_{0} ; W^{3, q}(\Omega)\right), \quad \partial_{t}(\mathbf{d}-\overline{\mathbf{d}}) \in L^{p}\left(0, T_{0} ; L^{q}(\Omega)\right), \\
P \in L^{p}\left(0, T_{0} ; W^{1, q}(\Omega)\right) .
\end{gathered}
$$

We claim that all the nonlinear terms in (4.1), (4.2), (4.3) converge to their corresponding terms in (1.1) almost everywhere in $\Omega \times\left(0, T_{0}\right)$. Indeed, for $\alpha:=$ $\frac{r s}{r+s}\left(=\frac{q r}{2 q+r}\right)$,

$$
\begin{aligned}
& \left\|\mathbf{u}^{n} \cdot \nabla \rho^{n+1}-\mathbf{u} \cdot \nabla \rho\right\|_{L_{T_{0}}^{\infty}\left(L^{\alpha}\right)} \\
& \leq\left\|\mathbf{u}^{n}-\mathbf{u}\right\|_{L_{T_{0}}^{\infty}\left(L^{s}\right)}\left\|\nabla \rho^{n+1}\right\|_{L_{T_{0}}^{\infty}\left(L^{r}\right)}+\|\mathbf{u}\|_{L_{T_{0}}^{\infty}\left(L^{s}\right)}\left\|\nabla \rho^{n+1}-\nabla \rho\right\|_{L_{T_{0}}^{\infty}\left(L^{r}\right)} \\
& \leq C\left(\varrho_{0}\left\|\mathbf{u}^{n}-\mathbf{u}\right\|_{M_{T_{0}}^{p, s, r}}+\|\mathbf{u}\|_{L_{T_{0}}^{\infty}\left(L^{s}\right)}\left\|\rho^{n+1}-\rho\right\|_{M_{T_{0}}^{p, s, r}}\right) \\
& \quad \rightarrow 0 \text { as } n \rightarrow \infty \\
& \left\|\rho^{n+1} \partial_{t} \mathbf{u}^{n+1}-\rho \partial_{t} \mathbf{u}\right\|_{L_{T_{0}}^{p}\left(L^{\alpha}\right)} \\
& \leq\left\|\rho^{n+1}\right\|_{L_{T_{0}}^{\infty}\left(L^{r}\right)}\left\|\partial_{t} \mathbf{u}^{n+1}-\partial_{t} \mathbf{u}\right\|_{L_{T_{0}}^{p}\left(L^{s}\right)}+\left\|\rho^{n+1}-\rho\right\|_{L_{T_{0}}^{\infty}\left(L^{r}\right)}\left\|\partial_{t} \mathbf{u}\right\|_{L_{T_{0}}^{p}\left(L^{s}\right)} \\
& \leq C\left(\varrho_{0}\left\|\mathbf{u}^{n}-\mathbf{u}\right\|_{M_{T_{0}}^{p, s, r}}+\left\|\partial_{t} \mathbf{u}\right\|_{L_{T_{0}}^{p}\left(L^{s}\right)}\left\|\rho^{n+1}-\rho\right\|_{M_{T_{0}}^{p, s, r}}\right) \\
& \rightarrow 0 \text { as } n \rightarrow \infty
\end{aligned}
$$

and

$$
\begin{aligned}
& \left\|\rho^{n+1} \mathbf{u}^{n} \cdot \nabla \mathbf{u}^{n}-\rho \mathbf{u} \cdot \nabla \mathbf{u}\right\|_{L_{T_{0}}^{p}\left(L^{\alpha}\right)} \\
& \leq\left\|\rho^{n+1}-\rho\right\|_{L_{T_{0}}^{\infty}\left(L^{r}\right)}\left\|\mathbf{u}^{n}\right\|_{L_{T_{0}}^{\infty}\left(L^{s}\right)}\left\|\nabla \mathbf{u}^{n}\right\|_{L_{T_{0}}^{p}\left(L^{\infty}\right)} \\
& +\|\rho\|_{L_{T_{0}}^{\infty}\left(L^{\infty}\right)}\left\|\mathbf{u}^{n}\right\|_{L_{T}^{\infty}\left(L^{s}\right)}\left\|\nabla \mathbf{u}^{n}-\nabla \mathbf{u}\right\|_{L_{T}^{p}\left(L^{r}\right)} \\
& +\|\rho\|_{L_{T_{0}}^{\infty}\left(L^{\infty}\right)}\left\|\mathbf{u}^{n}-\mathbf{u}\right\|_{L_{T_{0}}^{\infty}\left(L^{s}\right)}\|\nabla \mathbf{u}\|_{L_{T_{0}}^{p}\left(L^{r}\right)} \\
& \leq C\left\|\rho^{n+1}-\rho\right\|_{L_{T_{0}}^{\infty}\left(L^{r}\right)}\left\|\mathbf{u}^{n}\right\|_{L_{T_{0}}^{\infty}\left(L^{q}\right)}\left\|\mathbf{u}^{n}\right\|_{L_{T_{0}}^{p}\left(W^{2, q}\right)} \\
& +C\|\rho\|_{L_{T_{0}}^{\infty}\left(L^{\infty}\right)}\left\|\mathbf{u}^{n}\right\|_{L_{T_{0}}^{\infty}\left(L^{q}\right)}\left\|\nabla \mathbf{u}^{n}-\nabla \mathbf{u}\right\|_{L_{T_{0}}^{p}\left(L^{r}\right)} \\
& +\|\rho\|_{L_{T_{0}}^{\infty}\left(L^{\infty}\right)}\left\|\mathbf{u}^{n}-\mathbf{u}\right\|_{L_{T_{0}}^{\infty}\left(L^{s}\right)}\|\nabla \mathbf{u}\|_{L_{T_{0}}^{p}\left(L^{r}\right)} \\
& \leq C\left(\left(U^{0}\right)^{2}\left\|\rho^{n+1}-\rho\right\|_{M_{T_{0}}^{p, s, r}}+U^{0} T_{0}^{\frac{1}{2}-\frac{3}{2 q}}\|\rho\|_{L_{T_{0}}^{\infty}\left(L^{\infty}\right)}\left\|\mathbf{u}^{n}-\mathbf{u}\right\|_{M_{T_{0}}^{p, s, r}}\right. \\
& \left.+T_{0}^{\frac{1}{2}-\frac{3}{2 q}}\|\rho\|_{L_{T_{0}}^{\infty}\left(L^{\infty}\right)}\left\|\mathbf{u}^{n}-\mathbf{u}\right\|_{M_{T_{0}}^{p, s, r}}\|\mathbf{u}\|_{M_{T_{0}}^{p, s, r}}\right) \\
& \rightarrow 0 \text { as } n \rightarrow \infty \text {, }
\end{aligned}
$$

due to $\mathbf{u}^{n} \rightarrow \mathbf{u}$ and $\rho^{n+1} \rightarrow \rho$ in $M_{T_{0}}^{p, s, r}$ as $n \rightarrow \infty$. Hence,

$$
\begin{aligned}
\mathbf{u}^{n} \cdot \nabla \rho^{n+1} & \rightarrow \mathbf{u} \cdot \nabla \rho \quad \text { in } \quad L^{p}\left(0, T_{0} ; L^{\alpha}(\Omega)\right) ; \\
\rho^{n+1} \partial_{t} \mathbf{u}^{n+1} & \rightarrow \rho \partial_{t} \mathbf{u} \quad \text { in } \quad\left(L^{p}\left(0, T_{0} ; L^{\alpha}(\Omega)\right)\right)^{3} ; \\
\rho^{n+1} \mathbf{u}^{n} \cdot \nabla \mathbf{u}^{n} & \rightarrow \rho \mathbf{u} \cdot \nabla \mathbf{u} \quad \text { in } \quad\left(L^{p}\left(0, T_{0} ; L^{\alpha}(\Omega)\right)\right)^{3} .
\end{aligned}
$$


Meanwhile,

$$
\begin{aligned}
& \left\|\nabla \cdot\left(\left(\nabla\left(\mathbf{d}^{n}-\overline{\mathbf{d}}\right)\right)^{\top} \nabla\left(\mathbf{d}^{n}-\overline{\mathbf{d}}\right)\right)-\nabla \cdot\left((\nabla(\mathbf{d}-\overline{\mathbf{d}}))^{\top} \nabla(\mathbf{d}-\overline{\mathbf{d}})\right)\right\|_{L_{T_{0}}^{p}}\left(L^{\alpha}\right) \\
& =\| \frac{1}{2} \nabla\left(\left(\nabla\left(\mathbf{d}^{n}-\overline{\mathbf{d}}\right)+\nabla(\mathbf{d}-\overline{\mathbf{d}})\right):\left(\nabla \mathbf{d}^{n}-\nabla \mathbf{d}\right)\right)+\left(\nabla \mathbf{d}^{n}-\nabla \mathbf{d}\right)^{\top} \triangle\left(\mathbf{d}^{n}-\overline{\mathbf{d}}\right) \\
& +(\nabla(\mathbf{d}-\overline{\mathbf{d}}))^{\top}\left(\triangle \mathbf{d}^{n}-\triangle \mathbf{d}\right) \|_{L_{T_{0}}^{p}\left(L^{\alpha}\right)} \\
& \leq C\left(\left\|\nabla \mathbf{d}^{n}-\nabla \mathbf{d}\right\|_{L_{T_{0}}^{\infty}\left(L^{s}\right)}\left\|\triangle\left(\mathbf{d}^{n}-\overline{\mathbf{d}}\right)+\triangle(\mathbf{d}-\overline{\mathbf{d}})\right\|_{L_{T_{0}}^{p}\left(L^{r}\right)}\right. \\
& +\left\|\nabla\left(\mathbf{d}^{n}-\overline{\mathbf{d}}\right)+\nabla(\mathbf{d}-\overline{\mathbf{d}})\right\|_{L_{T_{0}}^{\infty}\left(L^{s}\right)}\left\|\Delta \mathbf{d}^{n}-\triangle \mathbf{d}\right\|_{L_{T_{0}}^{p}\left(L^{r}\right)} \\
& +\left\|\nabla \mathbf{d}^{n}-\nabla \mathbf{d}\right\|_{L_{T_{0}}^{\infty}\left(L^{s}\right)}\left\|\triangle\left(\mathbf{d}^{n}-\overline{\mathbf{d}}\right)\right\|_{L_{T_{0}}^{p}\left(L^{r}\right)} \\
& \left.+\|\nabla(\mathbf{d}-\overline{\mathbf{d}})\|_{L_{T_{0}}^{\infty}\left(L^{s}\right)}\left\|\triangle \mathbf{d}^{n}-\triangle \mathbf{d}\right\|_{L_{T_{0}}^{p}\left(L^{r}\right)}\right) \\
& \leq C T_{0}^{\frac{1}{3}-\frac{1}{q}}\left(U^{0}+\|\mathbf{d}-\overline{\mathbf{d}}\|_{M_{T_{0}}^{p, s, r}}\right)\left\|\mathbf{d}^{n}-\mathbf{d}\right\|_{M_{T_{0}}^{p, s, r}} \\
& \rightarrow 0 \text { as } n \rightarrow \infty \text {. }
\end{aligned}
$$

Moreover,

$$
\begin{aligned}
& \left\|\mathbf{u}^{n} \cdot \nabla\left(\mathbf{d}^{n}-\overline{\mathbf{d}}\right)-\mathbf{u} \cdot \nabla(\mathbf{d}-\overline{\mathbf{d}})\right\|_{L_{T_{0}}^{p}\left(L^{s}\right)} \\
& \leq\left\|\mathbf{u}^{n}-\mathbf{u}\right\|_{L_{T_{0}}^{\infty}\left(L^{s}\right)}\left\|\nabla\left(\mathbf{d}^{n}-\overline{\mathbf{d}}\right)\right\|_{L_{T_{0}}^{p}\left(L^{\infty}\right)}+\|\mathbf{u}\|_{L_{T_{0}}^{\infty}\left(L^{s}\right)}\left\|\nabla \mathbf{d}^{n}-\nabla \mathbf{d}\right\|_{L_{T_{0}}^{p}\left(L^{\infty}\right)} \\
& \leq C\left(U^{0}\left\|\mathbf{u}^{n}-\mathbf{u}\right\|_{M_{T_{0}}^{p, s, r}}+\|\mathbf{u}\|_{L_{T_{0}}^{\infty}\left(L^{s}\right)}\left\|\mathbf{d}^{n}-\mathbf{d}\right\|_{M_{T_{0}}^{p, s, r}}\right) \\
& \rightarrow 0 \text { as } n \rightarrow \infty
\end{aligned}
$$

$$
\begin{aligned}
& \left\|\left|\nabla\left(\mathbf{d}^{n}-\overline{\mathbf{d}}\right)\right|^{2} \overline{\mathbf{d}}-|\nabla(\mathbf{d}-\overline{\mathbf{d}})|^{2} \overline{\mathbf{d}}\right\|_{L_{T_{0}}^{p}\left(L^{s}\right)} \\
& \leq C\left\|\nabla\left(\mathbf{d}^{n}-\overline{\mathbf{d}}\right)+\nabla(\mathbf{d}-\overline{\mathbf{d}})\right\|_{L_{T_{0}}^{\infty}\left(L^{s}\right)}\left\|\nabla \mathbf{d}^{n}-\nabla \mathbf{d}\right\|_{L_{T_{0}}^{p}\left(L^{\infty}\right)} \\
& \leq C\left(U^{0}+\|\mathbf{d}-\overline{\mathbf{d}}\|_{\left.M_{T_{0}}^{p, s, r}\right)\left\|\nabla \mathbf{d}^{n}-\nabla \mathbf{d}\right\|_{L_{T_{0}}^{p}\left(W^{2, s}\right)}}\right. \\
& \leq C\left(U^{0}+\|\mathbf{d}-\overline{\mathbf{d}}\|_{\left.M_{T_{0}}^{p, s, r}\right)\left\|\mathbf{d}^{n}-\mathbf{d}\right\|_{M_{T_{0}}^{p, s, r}}}\right. \\
& \rightarrow 0 \text { as } n \rightarrow \infty
\end{aligned}
$$

Then, we have

$$
\begin{gathered}
\nabla \cdot\left(\left(\nabla\left(\mathbf{d}^{n}-\overline{\mathbf{d}}\right)\right)^{\top} \nabla\left(\mathbf{d}^{n}-\overline{\mathbf{d}}\right)\right) \rightarrow \nabla \cdot\left((\nabla(\mathbf{d}-\overline{\mathbf{d}}))^{\top} \nabla(\mathbf{d}-\overline{\mathbf{d}})\right) \quad \text { in }\left(L^{p}\left(0, T_{0} ; L^{\alpha}(\Omega)\right)\right)^{3} ; \\
\mathbf{u}^{n} \cdot \nabla\left(\mathbf{d}^{n}-\overline{\mathbf{d}}\right) \rightarrow \mathbf{u} \cdot \nabla(\mathbf{d}-\overline{\mathbf{d}}) \quad \text { in }\left(L^{p}\left(0, T_{0} ; L^{s}(\Omega)\right)\right)^{3} ; \\
\left|\nabla\left(\mathbf{d}^{n}-\overline{\mathbf{d}}\right)\right|^{2} \overline{\mathbf{d}} \rightarrow|\nabla(\mathbf{d}-\overline{\mathbf{d}})|^{2} \overline{\mathbf{d}} \quad \text { in }\left(L^{p}\left(0, T_{0} ; L^{s}(\Omega)\right)\right)^{3} .
\end{gathered}
$$

We know $\mathbf{d}-\overline{\mathbf{d}} \in C\left(\left[0, T_{0}\right] ; B_{s, p}^{3\left(1-\frac{1}{p}\right)}\right) \cap\left(L^{p}\left(0, T_{0} ; W^{3, s}(\Omega)\right)\right)^{3}, s=\frac{q r}{q+r}>\frac{3}{2}$, and $W^{1, s} \hookrightarrow L^{\infty}$ if $s>3$. As for $s=3, W^{1, s} \hookrightarrow L^{\infty-}$ with $\infty$ - denoting any positive number large enough (but not $\infty$ ), we will use $W^{1, s} \hookrightarrow L^{\frac{3 q}{9-q}}$ below. When $\frac{3}{2}<s<3, W^{2, s} \hookrightarrow L^{\infty}$. Due to these different embedding properties, we discuss the term $\left|\nabla\left(\mathbf{d}^{n}-\overline{\mathbf{d}}\right)\right|^{2}\left(\mathbf{d}^{n}-\overline{\mathbf{d}}\right)$ respectively for $s>3, \frac{3}{2}<s<3$ and $s=3$. 
If $s>3$, we have

$$
\begin{aligned}
& \left\|\left|\nabla\left(\mathbf{d}^{n}-\overline{\mathbf{d}}\right)\right|^{2}\left(\mathbf{d}^{n}-\overline{\mathbf{d}}\right)-|\nabla(\mathbf{d}-\overline{\mathbf{d}})|^{2}(\mathbf{d}-\overline{\mathbf{d}})\right\|_{L_{T_{0}}^{p}\left(L^{\frac{q}{3}}\right)} \\
& \leq\left\|\nabla\left(\mathbf{d}^{n}-\overline{\mathbf{d}}\right)\right\|_{L_{T_{0}}^{\infty}\left(L^{q}\right)}\left(\left\|\nabla\left(\mathbf{d}^{n}-\overline{\mathbf{d}}\right)\right\|_{L_{T_{0}}^{p}\left(L^{\infty}\right)}\left\|\mathbf{d}^{n}-\mathbf{d}\right\|_{L_{T_{0}}^{\infty}\left(L^{\frac{q}{2}}\right)}\right. \\
& \left.+\|\mathbf{d}-\overline{\mathbf{d}}\|_{L_{T_{0}}^{p}\left(L^{\infty}\right)}\left\|\nabla \mathbf{d}^{n}-\nabla \mathbf{d}\right\|_{L_{T_{0}}^{\infty}\left(L^{\frac{q}{2}}\right)}\right) \\
& +\|\mathbf{d}-\overline{\mathbf{d}}\|_{L_{T_{0}}^{\infty}\left(L^{\infty}\right)}\|\nabla(\mathbf{d}-\overline{\mathbf{d}})\|_{L_{T_{0}}^{p}\left(L^{q}\right)}\left\|\nabla \mathbf{d}^{n}-\nabla \mathbf{d}\right\|_{L_{T_{0}}^{\infty}\left(L^{\frac{q}{2}}\right)} \\
& \leq C\left\|\nabla\left(\mathbf{d}^{n}-\overline{\mathbf{d}}\right)\right\|_{L_{T_{0}}^{\infty}\left(L^{q}\right)}\left(\left\|\nabla\left(\mathbf{d}^{n}-\overline{\mathbf{d}}\right)\right\|_{L_{T_{0}}^{p}\left(W^{1, q}\right)}\left\|\mathbf{d}^{n}-\mathbf{d}\right\|_{L_{T_{0}}^{\infty}\left(L^{s}\right)}\right. \\
& \left.+\|\mathbf{d}-\overline{\mathbf{d}}\|_{\left.L_{T_{0}}^{p}\left(W^{1, s}\right)\right)}\left\|\nabla \mathbf{d}^{n}-\nabla \mathbf{d}\right\|_{L_{T_{0}}^{\infty}\left(L^{s}\right)}\right) \\
& +C\|\mathbf{d}-\overline{\mathbf{d}}\|_{\left.L_{T_{0}}^{\infty}\left(W^{1, s}\right)\right)}\|\nabla(\mathbf{d}-\overline{\mathbf{d}})\|_{L_{T_{0}}^{p}\left(L^{r}\right)}\left\|\nabla \mathbf{d}^{n}-\nabla \mathbf{d}\right\|_{L_{T_{0}}^{\infty}\left(L^{s}\right)} \\
& \leq C U^{0}\left(U^{0}+\|\mathbf{d}-\overline{\mathbf{d}}\|_{M_{T_{0}}^{p, s, r}}\right)\left\|\mathbf{d}^{n}-\mathbf{d}\right\|_{M_{T_{0}}^{p, s, r}}+C T_{0}^{\frac{2}{3}-\frac{1}{q}}\|\mathbf{d}-\overline{\mathbf{d}}\|_{M_{T_{0}}^{p, s, r}}^{2}\left\|\mathbf{d}^{n}-\mathbf{d}\right\|_{M_{T_{0}}^{p, s, r}} \\
& \rightarrow 0 \text { as } n \rightarrow \infty \text {. }
\end{aligned}
$$

If $\frac{3}{2}<s<3$, we have

$$
\begin{aligned}
& \left\|\left|\nabla\left(\mathbf{d}^{n}-\overline{\mathbf{d}}\right)\right|^{2}\left(\mathbf{d}^{n}-\overline{\mathbf{d}}\right)-|\nabla(\mathbf{d}-\overline{\mathbf{d}})|^{2}(\mathbf{d}-\overline{\mathbf{d}})\right\|_{L_{T_{0}}^{p}\left(L^{\frac{q}{3}}\right)} \\
& \leq C\left(\left\|\left|\nabla\left(\mathbf{d}^{n}-\overline{\mathbf{d}}\right)\right|^{2}\left(\mathbf{d}^{n}-\mathbf{d}\right)\right\|_{L_{T_{0}}^{p}\left(L^{\frac{q}{3}}\right)}\right. \\
& \left.+\left\|\nabla\left(\mathbf{d}^{n}-\overline{\mathbf{d}}\right):\left(\nabla \mathbf{d}^{n}-\nabla \mathbf{d}\right)(\mathbf{d}-\overline{\mathbf{d}})\right\|_{L_{T_{0}}^{p}\left(L^{\frac{q}{3}}\right)}\right) \\
& +\left\|\nabla(\mathbf{d}-\overline{\mathbf{d}}):\left(\nabla \mathbf{d}^{n}-\nabla \mathbf{d}\right)(\mathbf{d}-\overline{\mathbf{d}})\right\|_{L_{T_{0}}^{p}\left(L^{\frac{3 s}{6-s}}\right)} \\
& \leq C\left\|\nabla\left(\mathbf{d}^{n}-\overline{\mathbf{d}}\right)\right\|_{L_{T_{0}}^{\infty}\left(L^{q}\right)}\left(\left\|\nabla\left(\mathbf{d}^{n}-\overline{\mathbf{d}}\right)\right\|_{L_{T_{0}}^{p}\left(L^{\infty}\right)}\left\|\mathbf{d}^{n}-\mathbf{d}\right\|_{L_{T_{0}}^{\infty}\left(L^{\frac{q}{2}}\right)}\right. \\
& \left.+\|\mathbf{d}-\overline{\mathbf{d}}\|_{L_{T_{0}}^{p}\left(L^{\infty}\right)}\left\|\nabla \mathbf{d}^{n}-\nabla \mathbf{d}\right\|_{L_{T_{0}}^{\infty}\left(L^{\frac{q}{2}}\right)}\right) \\
& +\|\mathbf{d}-\overline{\mathbf{d}}\|_{L_{T_{0}}^{\infty}\left(L^{\frac{3 s}{3-s}}\right)}\|\nabla(\mathbf{d}-\overline{\mathbf{d}})\|_{L_{T_{0}}^{p}\left(L^{\infty}\right)}\left\|\nabla \mathbf{d}^{n}-\nabla \mathbf{d}\right\|_{L_{T_{0}}^{\infty}\left(L^{s}\right)} \\
& \leq C\left\|\nabla\left(\mathbf{d}^{n}-\overline{\mathbf{d}}\right)\right\|_{L_{T_{0}}^{\infty}\left(L^{q}\right)}\left(\left\|\nabla\left(\mathbf{d}^{n}-\overline{\mathbf{d}}\right)\right\|_{L_{T_{0}}^{p}\left(W^{1, q}\right)}\left\|\mathbf{d}^{n}-\mathbf{d}\right\|_{L_{T_{0}}^{\infty}\left(L^{s}\right)}\right. \\
& \left.+\|\mathbf{d}-\overline{\mathbf{d}}\|_{\left.L_{T_{0}}^{p}\left(W^{2, s}\right)\right)}\left\|\nabla \mathbf{d}^{n}-\nabla \mathbf{d}\right\|_{L_{T_{0}}^{\infty}\left(L^{s}\right)}\right) \\
& +C\|\mathbf{d}-\overline{\mathbf{d}}\|_{\left.L_{T_{0}}^{\infty}\left(W^{1, s}\right)\right)}\|\nabla(\mathbf{d}-\overline{\mathbf{d}})\|_{L_{T_{0}}^{p}\left(W^{2, s}\right)}\left\|\nabla \mathbf{d}^{n}-\nabla \mathbf{d}\right\|_{L_{T_{0}}^{\infty}\left(L^{s}\right)} \\
& \leq C U^{0}\left(U^{0}+\|\mathbf{d}-\overline{\mathbf{d}}\|_{M_{T_{0}}^{p, s, r}}\right)\left\|\mathbf{d}^{n}-\mathbf{d}\right\|_{M_{T_{0}}^{p, s, r}}+C\|\mathbf{d}-\overline{\mathbf{d}}\|_{M_{T_{0}}^{p, s, r}}^{2}\left\|\mathbf{d}^{n}-\mathbf{d}\right\|_{M_{T_{0}}^{p, s, r}} \\
& \rightarrow 0 \text { as } n \rightarrow \infty \text {. }
\end{aligned}
$$


The case $s=3$ may be handled by noticing that we also have

$$
\begin{aligned}
& \left\|\left|\nabla\left(\mathbf{d}^{n}-\overline{\mathbf{d}}\right)\right|^{2}\left(\mathbf{d}^{n}-\overline{\mathbf{d}}\right)-|\nabla(\mathbf{d}-\overline{\mathbf{d}})|^{2}(\mathbf{d}-\overline{\mathbf{d}})\right\|_{L_{T_{0}}^{p}\left(L^{\frac{q}{3}}\right)} \\
& \leq\left\|\nabla\left(\mathbf{d}^{n}-\overline{\mathbf{d}}\right)\right\|_{L_{T_{0}}^{\infty}\left(L^{q}\right)}\left(\left\|\nabla\left(\mathbf{d}^{n}-\overline{\mathbf{d}}\right)\right\|_{L_{T_{0}}^{p}\left(L^{\infty}\right)}\left\|\mathbf{d}^{n}-\mathbf{d}\right\|_{L_{T_{0}}^{\infty}\left(L^{\frac{q}{2}}\right)}\right. \\
& \left.+\|\mathbf{d}-\overline{\mathbf{d}}\|_{L_{T_{0}}^{p}\left(L^{\infty}\right)}\left\|\nabla \mathbf{d}^{n}-\nabla \mathbf{d}\right\|_{L_{T_{0}}^{\infty}\left(L^{\frac{q}{2}}\right)}\right) \\
& +\|\mathbf{d}-\overline{\mathbf{d}}\|_{L_{T_{0}}^{\infty}\left(L^{\frac{3 q}{9-q}}\right)}\|\nabla(\mathbf{d}-\overline{\mathbf{d}})\|_{L_{T_{0}}^{p}\left(L^{\infty}\right)}\left\|\nabla \mathbf{d}^{n}-\nabla \mathbf{d}\right\|_{L_{T_{0}}^{\infty}\left(L^{3}\right)} \\
& \leq C\left\|\nabla\left(\mathbf{d}^{n}-\overline{\mathbf{d}}\right)\right\|_{L_{T_{0}}^{\infty}\left(L^{q}\right)}\left(\left\|\nabla\left(\mathbf{d}^{n}-\overline{\mathbf{d}}\right)\right\|_{L_{T_{0}}^{p}\left(W^{1, q}\right)}\left\|\mathbf{d}^{n}-\mathbf{d}\right\|_{L_{T_{0}}^{\infty}\left(L^{3}\right)}\right. \\
& \left.+\|\mathbf{d}-\overline{\mathbf{d}}\|_{\left.L_{T_{0}}^{p}\left(W^{2,3}\right)\right)}\left\|\nabla \mathbf{d}^{n}-\nabla \mathbf{d}\right\|_{L_{T_{0}}^{\infty}\left(L^{3}\right)}\right) \\
& +C\|\mathbf{d}-\overline{\mathbf{d}}\|_{L_{T_{0}}^{\infty}\left(W^{1,3}\right)}\|\nabla(\mathbf{d}-\overline{\mathbf{d}})\|_{L_{T_{0}}^{p}\left(W^{2,3}\right)}\left\|\nabla \mathbf{d}^{n}-\nabla \mathbf{d}\right\|_{L_{T_{0}}^{\infty}\left(L^{3}\right)} \\
& \leq C U^{0}\left(U^{0}+\|\mathbf{d}-\overline{\mathbf{d}}\|_{M_{T_{0}}^{p, 3, r}}\right)\left\|\mathbf{d}^{n}-\mathbf{d}\right\|_{M_{T_{0}}^{p, 3, r}}+C\|\mathbf{d}-\overline{\mathbf{d}}\|_{M_{T_{0}}^{p, 3, r}}^{2}\left\|\mathbf{d}^{n}-\mathbf{d}\right\|_{M_{T_{0}}^{p, 3, r}} \\
& \rightarrow 0 \text { as } n \rightarrow \infty \text {. }
\end{aligned}
$$

Hence, we finally get

$$
\left|\nabla\left(\mathbf{d}^{n}-\overline{\mathbf{d}}\right)\right|^{2}\left(\mathbf{d}^{n}-\overline{\mathbf{d}}\right) \rightarrow|\nabla(\mathbf{d}-\overline{\mathbf{d}})|^{2}(\mathbf{d}-\overline{\mathbf{d}}) \quad \text { in }\left(L^{p}\left(0, T_{0} ; L^{\frac{q}{3}}(\Omega)\right)\right)^{3} .
$$

Thus, passing to the limit in (4.1), (4.2) and (4.3) as $n \rightarrow \infty$, since $L^{s}(\Omega) \hookrightarrow$ $L^{\alpha}(\Omega) \hookrightarrow L^{\frac{q}{3}}(\Omega)$, we conclude that (1.1) holds in $L^{p}\left(0, T_{0} ; L^{\frac{q}{3}}(\Omega)\right)$ or $\left(L^{p}\left(0, T_{0} ; L^{\frac{q}{3}}(\Omega)\right)\right)^{3}$, and therefore almost everywhere in $\Omega \times\left(0, T_{0}\right)$.

Next, we check that $\mathbf{d} \in \mathbb{S}^{2}$. Multiplying the $\mathbf{d}$-system (1.1c) by $\mathbf{d}$, we obtain

$$
\frac{1}{2} \partial_{t}\left(|\mathbf{d}|^{2}\right)+\frac{1}{2} \mathbf{u} \cdot \nabla\left(|\mathbf{d}|^{2}\right)=\triangle \mathbf{d} \cdot \mathbf{d}+|\nabla \mathbf{d}|^{2}|\mathbf{d}|^{2} .
$$

Since

$$
\triangle\left(|\mathbf{d}|^{2}\right)=2|\nabla \mathbf{d}|^{2}+2 \mathbf{d} \cdot(\triangle \mathbf{d})
$$

it follows that

$$
\frac{1}{2} \partial_{t}\left(|\mathbf{d}|^{2}\right)+\frac{1}{2} \mathbf{u} \cdot \nabla\left(|\mathbf{d}|^{2}\right)=\frac{1}{2} \triangle\left(|\mathbf{d}|^{2}\right)-|\nabla \mathbf{d}|^{2}+|\nabla \mathbf{d}|^{2}|\mathbf{d}|^{2} .
$$

Therefore, it is easy to deduce that

$$
\partial_{t}\left(|\mathbf{d}|^{2}-1\right)-\triangle\left(|\mathbf{d}|^{2}-1\right)+\mathbf{u} \cdot \nabla\left(|\mathbf{d}|^{2}-1\right)-2|\nabla \mathbf{d}|^{2}\left(|\mathbf{d}|^{2}-1\right)=0 .
$$

Multiplying (4.29) by $\left(|\mathbf{d}|^{2}-1\right)$ and then integrating over $\Omega$, using (1.1d) and (1.3), we get the following inequality:

$$
\begin{aligned}
\frac{d}{d t} \int_{\Omega}\left(|\mathbf{d}|^{2}-1\right)^{2} d \mathbf{x} & \leq 4 \int_{\Omega}|\nabla \mathbf{d}|^{2}\left(|\mathbf{d}|^{2}-1\right)^{2} d \mathbf{x} \\
& \leq 4\|\nabla \mathbf{d}\|_{L^{\infty}}^{2} \int_{\Omega}\left(|\mathbf{d}|^{2}-1\right)^{2} d \mathbf{x} .
\end{aligned}
$$


Note that interpolation between $L^{\infty}\left(0, T_{0} ; W^{1, q}(\Omega)\right)$ and $L^{p}\left(0, T_{0} ; W^{3, q}(\Omega)\right)$ shows that for some positive $\alpha>\frac{1}{2}, \mathbf{d}-\overline{\mathbf{d}}$ belongs to $\left(L^{2}\left(0, T_{0} ; H^{2+\alpha}(\Omega)\right)\right)^{3}$ and that $\|\nabla \mathbf{d}\|_{L^{\infty}}^{2} \in L^{1}\left(0, T_{0}\right)$. Notice also that

$$
\int_{\Omega}\left(|\mathbf{d}|^{2}-1\right)^{2} d \mathbf{x}=0, \quad \text { at time } t=0
$$

Thus, using (4.30) together with Grönwall's inequality yields $|\mathbf{d}|=1$ in $\Omega \times\left(0, T_{0}\right)$.

4.5. Uniqueness and continuity. Let $\left(\rho_{1}, \mathbf{u}_{1}, P_{1}, \mathbf{d}_{1}\right)$ and $\left(\rho_{2}, \mathbf{u}_{2}, P_{2}, \mathbf{d}_{2}\right)$ be two solutions to (1.1) with the initial-boundary conditions (1.2), (1.3). Denote

$$
\bar{\rho}=\rho_{1}-\rho_{2}, \quad \overline{\mathbf{u}}=\mathbf{u}_{1}-\mathbf{u}_{2}, \quad \bar{P}=P_{1}-P_{2}, \quad \overline{\mathbf{d}}=\mathbf{d}_{1}-\mathbf{d}_{2} .
$$

Note that the quadruplet $(\bar{\rho}, \overline{\mathbf{u}}, \bar{P}, \overline{\mathbf{d}})$ satisfies the following system:

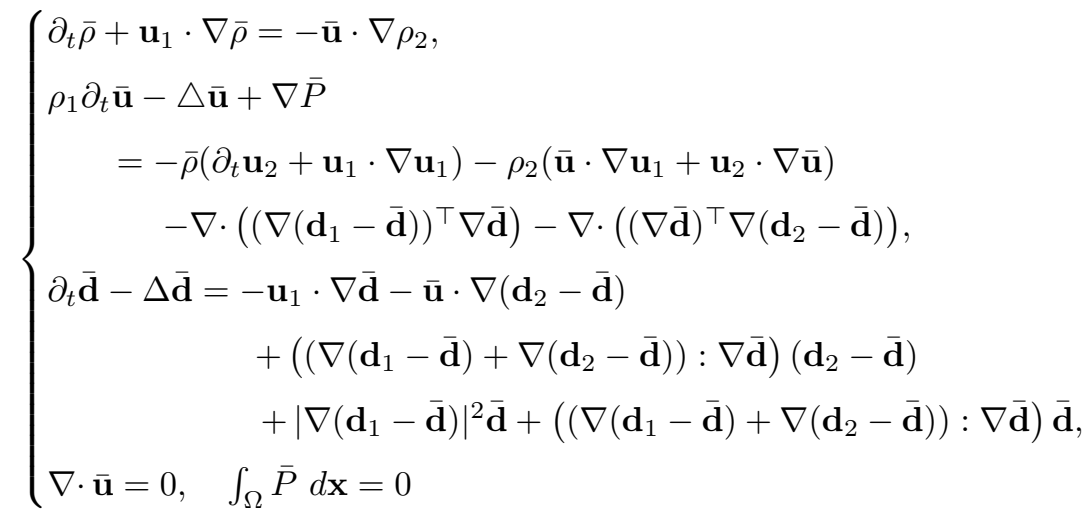

with the initial-boundary conditions

$$
\left.(\bar{\rho}, \overline{\mathbf{u}}, \overline{\mathbf{d}})\right|_{t=0}=(0,0,0),\left.\quad\left(\overline{\mathbf{u}}, \partial_{\nu} \overline{\mathbf{d}}\right)\right|_{\partial \Omega}=(0,0) .
$$

Using the same argument for $\bar{\rho}^{n}$ in Subsection 4.3, for all $t \in\left[0, T_{0}\right]$, we have

$$
\begin{aligned}
\|\bar{\rho}(t)\|_{L^{r}} & \leq \int_{0}^{t}\left\|\nabla \rho_{2}(\tau)\right\|_{L^{r}}\|\overline{\mathbf{u}}(\tau)\|_{L^{\infty}} d \tau \\
& \leq t^{1-\frac{1}{p}}\left\|\nabla \rho_{2}\right\|_{L_{t}^{\infty}\left(L^{r}\right)}\|\overline{\mathbf{u}}\|_{L_{t}^{p}\left(L^{\infty}\right)} \\
& \leq C t^{1-\frac{1}{p}}\left\|\rho_{2}\right\|_{L_{t}^{\infty}\left(W^{1, r}\right)}\|\overline{\mathbf{u}}\|_{L_{t}^{p}\left(W^{2, s}\right)} .
\end{aligned}
$$

On the one hand, since $\rho_{1}, \rho_{2} \in L^{\infty}\left(0, T_{0} ; W^{1, r}(\Omega)\right) \cap W^{1, \infty}\left(0, T_{0} ; L^{s}(\Omega)\right)$ implies that $\rho_{1}, \rho_{2} \in C^{\beta}\left(\left[0, T_{0}\right] ; L^{\infty}(\Omega)\right)$ whenever $\beta \in\left(0, \frac{1-\frac{3}{r}}{1+\frac{3}{q}}\right)$, Theorem 3.2 yields, for some constant $\mathrm{C}$ depending on $T_{0}, p, q, r, \check{\rho}, \hat{\rho}, \Omega, \beta$ and on the norm of $\rho_{1}$ in 
$L^{\infty}\left(0, T_{0} ; W^{1, r}(\Omega)\right) \cap C^{\beta}\left(0, T_{0} ; L^{\infty}(\Omega)\right)$, and for all $t \in\left[0, T_{0}\right]$,

$$
\begin{aligned}
& \|\overline{\mathbf{u}}(t)\|_{D_{A_{s}}^{1-\frac{1}{p}, p}}+\|\overline{\mathbf{u}}\|_{L_{t}^{p}\left(W^{2, s}\right)}+\left\|\partial_{t} \overline{\mathbf{u}}\right\|_{L_{t}^{p}\left(L^{s}\right)}+\|\bar{P}\|_{L_{t}^{p}\left(W^{1, s}\right)} \\
& \leq C\left(\left\|\bar{\rho}\left(\partial_{t} \mathbf{u}_{2}+\mathbf{u}_{1} \cdot \nabla \mathbf{u}_{1}\right)\right\|_{L_{t}^{p}\left(L^{s}\right)}+\left\|\rho_{2} \overline{\mathbf{u}} \cdot \nabla \mathbf{u}_{1}\right\|_{L_{t}^{p}\left(L^{s}\right)}+\left\|\rho_{2} \mathbf{u}_{2} \cdot \nabla \overline{\mathbf{u}}\right\|_{L_{t}^{p}\left(L^{s}\right)}\right. \\
& \left.+\left\|\nabla \cdot\left(\left(\nabla\left(\mathbf{d}_{1}-\overline{\mathbf{d}}\right)\right)^{\top} \nabla \overline{\mathbf{d}}\right)\right\|_{L_{t}^{p}\left(L^{s}\right)}+\left\|\nabla \cdot\left((\nabla \overline{\mathbf{d}})^{\top} \nabla\left(\mathbf{d}_{2}-\overline{\mathbf{d}}\right)\right)\right\|_{L_{t}^{p}\left(L^{s}\right)}\right) \\
& \leq C\left(\|\bar{\rho}\|_{L_{t}^{\infty}\left(L^{r}\right)}\left(\left\|\partial_{t} \mathbf{u}_{2}\right\|_{L_{t}^{p}\left(L^{q}\right)}+\left\|\mathbf{u}_{1}\right\|_{L_{t}^{\infty}\left(L^{q}\right)}\left\|\nabla \mathbf{u}_{1}\right\|_{L_{t}^{p}\left(L^{\infty}\right)}\right)\right. \\
& +\left\|\nabla \mathbf{u}_{1}\right\|_{L_{t}^{p}\left(L^{\infty}\right)}\|\overline{\mathbf{u}}\|_{L_{t}^{\infty}\left(L^{s}\right)} \\
& +\left\|\mathbf{u}_{2}\right\|_{L_{t}^{\infty}\left(L^{q}\right)}\|\nabla \overline{\mathbf{u}}\|_{L_{t}^{p}\left(L^{r}\right)}+\left\|\nabla\left(\mathbf{d}_{1}-\overline{\mathbf{d}}\right)\right\|_{L_{t}^{\infty}\left(L^{q}\right)}\|\triangle \overline{\mathbf{d}}\|_{L_{t}^{p}\left(L^{r}\right)} \\
& +\left\|\triangle\left(\mathbf{d}_{1}-\overline{\mathbf{d}}\right)\right\|_{L_{t}^{p}\left(L^{\infty}\right)}\|\nabla \overline{\mathbf{d}}\|_{L_{t}^{\infty}\left(L^{s}\right)}+\left\|\nabla\left(\mathbf{d}_{2}-\overline{\mathbf{d}}\right)\right\|_{L_{t}^{\infty}\left(L^{q}\right)}\|\triangle \overline{\mathbf{d}}\|_{L_{t}^{p}\left(L^{r}\right)} \\
& \left.+\left\|\triangle\left(\mathbf{d}_{2}-\overline{\mathbf{d}}\right)\right\|_{L_{t}^{p}\left(L^{\infty}\right)}\|\nabla \overline{\mathbf{d}}\|_{L_{t}^{\infty}\left(L^{s}\right)}\right) \\
& \leq C\left(\|\bar{\rho}\|_{L_{t}^{\infty}\left(L^{r}\right)}\left(\left\|\partial_{t} \mathbf{u}_{2}\right\|_{L_{t}^{p}\left(L^{q}\right)}+\left\|\mathbf{u}_{1}\right\|_{L_{t}^{\infty}\left(D_{A_{q}}^{1-\frac{1}{p}, p}\right)}\left\|\mathbf{u}_{1}\right\|_{L_{t}^{p}\left(W^{2, q}\right)}\right)\right. \\
& +\left\|\mathbf{u}_{1}\right\|_{L_{t}^{p}\left(W^{2, q}\right)}\|\overline{\mathbf{u}}\|_{L_{t}^{\infty}\left(L^{s}\right)}+\left\|\mathbf{u}_{2}\right\|_{L_{t}^{\infty}\left(D_{A_{q}}^{1-\frac{1}{p}, p}\right)}\|\nabla \overline{\mathbf{u}}\|_{L_{t}^{p}\left(L^{r}\right)} \\
& +\left(\left\|\mathbf{d}_{1}-\overline{\mathbf{d}}\right\|_{L_{t}^{\infty}\left(B_{q, p}^{3\left(1-\frac{1}{p}\right)}\right)}+\left\|\mathbf{d}_{2}-\overline{\mathbf{d}}\right\|_{L_{t}^{\infty}\left(B_{q, p}^{3\left(1-\frac{1}{p}\right)}\right)}\right)\|\Delta \overline{\mathbf{d}}\|_{L_{t}^{p}\left(L^{r}\right)} \\
& \left.+\left(\left\|\mathbf{d}_{1}-\overline{\mathbf{d}}\right\|_{L_{t}^{p}\left(W^{3, q}\right)}+\left\|\mathbf{d}_{2}-\overline{\mathbf{d}}\right\|_{L_{t}^{p}\left(W^{3, q}\right)}\right)\|\nabla \overline{\mathbf{d}}\|_{L_{t}^{\infty}\left(L^{s}\right)}\right) .
\end{aligned}
$$

On the other hand, Theorem 3.1 yields, for some constant $\mathrm{C}$ independent of $T_{0}$,

$$
\begin{aligned}
& \|\overline{\mathbf{d}}(t)\|_{B_{s, p}^{3\left(1-\frac{1}{p}\right)}}+\|\overline{\mathbf{d}}\|_{\mathcal{W}_{s, p}(0, t)} \\
& \leq C\left(\|-\mathbf{u}_{1} \cdot \nabla \overline{\mathbf{d}}-\overline{\mathbf{u}} \cdot \nabla\left(\mathbf{d}_{2}-\overline{\mathbf{d}}\right)+\left|\nabla\left(\mathbf{d}_{1}-\overline{\mathbf{d}}\right)\right|^{2} \overline{\mathbf{d}}\right. \\
& \quad+\left(\left(\nabla\left(\mathbf{d}_{1}-\overline{\mathbf{d}}\right)+\nabla\left(\mathbf{d}_{2}-\overline{\mathbf{d}}\right)\right): \nabla \overline{\mathbf{d}}\right)\left(\mathbf{d}_{2}-\overline{\mathbf{d}}\right) \\
& \left.\quad+\left(\left(\nabla\left(\mathbf{d}_{1}-\overline{\mathbf{d}}\right)+\nabla\left(\mathbf{d}_{2}-\overline{\mathbf{d}}\right)\right): \nabla \overline{\mathbf{d}}\right) \overline{\mathbf{d}} \|_{L_{t}^{p}\left(L^{s}\right)}\right) \\
& \quad+C\left(\left\|\mathbf{u}_{1}\right\|_{L_{t}^{\infty}\left(L^{q}\right)}\|\nabla \overline{\mathbf{d}}\|_{L_{t}^{p}\left(L^{r}\right)}+\left\|\nabla\left(\mathbf{d}_{2}-\overline{\mathbf{d}}\right)\right\|_{L_{t}^{p}\left(L^{\infty}\right)}\|\overline{\mathbf{u}}\|_{L_{t}^{\infty}\left(L^{s}\right)}\right. \\
& \quad+\left\|\nabla\left(\mathbf{d}_{1}-\overline{\mathbf{d}}\right)\right\|_{L_{t}^{\infty}\left(L^{q}\right)}\left\|\nabla\left(\mathbf{d}_{1}-\overline{\mathbf{d}}\right)\right\|_{L_{t}^{p}\left(L^{\infty}\right)}\|\overline{\mathbf{d}}\|_{L_{t}^{\infty}\left(L^{r}\right)} \\
& \left.\quad+\left(\left\|\nabla\left(\mathbf{d}_{1}-\overline{\mathbf{d}}\right)\right\|_{L_{t}^{p}\left(L^{\infty}\right)}+\left\|\nabla\left(\mathbf{d}_{2}-\overline{\mathbf{d}}\right)\right\|_{L_{t}^{p}\left(L^{\infty}\right)}\right)\|\nabla \overline{\mathbf{d}}\|_{L_{t}^{\infty}\left(L^{s}\right)}\left(\left\|\mathbf{d}_{2}-\overline{\mathbf{d}}\right\|_{L_{t}^{\infty}\left(L^{\infty}\right)}+1\right)\right) \\
& \leq C\left(\left.\left\|\mathbf{u}_{1}\right\|\right|_{L_{t}^{\infty}\left(D_{A_{q}}^{1-\frac{1}{p}, p}\right)}\|\nabla \overline{\mathbf{d}}\|_{L_{t}^{p}\left(L^{r}\right)}+\left\|\mathbf{d}_{2}-\overline{\mathbf{d}}\right\|_{L_{t}^{p}\left(W^{2, q}\right)}\|\overline{\mathbf{u}}\|_{L_{t}^{\infty}\left(L^{s}\right)}\right. \\
& \quad+\left\|\mathbf{d}_{1}-\overline{\mathbf{d}}\right\|_{L_{t}^{\infty}\left(B_{q, p}^{3\left(1-\frac{1}{p}\right)}\right)}\left\|\mathbf{d}_{1}-\overline{\mathbf{d}}\right\|_{L_{t}^{p}\left(W^{2, q}\right)}\|\overline{\mathbf{d}}\|_{L_{t}^{\infty}\left(L^{r}\right)} \\
& \left.\quad+\left(\left\|\mathbf{d}_{2}-\overline{\mathbf{d}}\right\|_{L_{t}^{\infty}\left(B_{q, p}^{3\left(1-\frac{1}{p}\right)}\right)}+1\right)\left(\left\|\mathbf{d}_{1}-\overline{\mathbf{d}}\right\|_{L_{t}^{p}\left(W^{2, q}\right)}+\left\|\mathbf{d}_{2}-\overline{\mathbf{d}}\right\|_{L_{t}^{p}\left(W^{2, q}\right)}\right)\|\nabla \overline{\mathbf{d}}\|_{L_{t}^{\infty}\left(L^{s}\right)}\right) .
\end{aligned}
$$

We remark here that Hölder's inequality and the embedding $W^{1, q}(\Omega) \hookrightarrow L^{\infty}(\Omega)$ $(q>3)$ have been employed repeatedly in both (4.32) and (4.33). 
Lemmas 3.1$] 3.3$ yield, by use of Young's inequality,

$$
\begin{aligned}
& \|\nabla \overline{\mathbf{u}}\|_{L_{t}^{p}\left(L^{r}\right)} \leq C t^{\frac{1}{2}-\frac{3}{2 q}}\left(\|\overline{\mathbf{u}}\|_{L_{t}^{\infty}\left(D_{A_{s}}^{1-\frac{1}{p}, p}\right)}+\|\overline{\mathbf{u}}\|_{L_{t}^{p}\left(W^{2, s}\right)}\right), \\
& \|\nabla \overline{\mathbf{d}}\|_{L_{t}^{p}\left(L^{r}\right)} \leq C t^{\frac{2}{3}-\frac{1}{q}}\left(\|\overline{\mathbf{d}}\|_{L_{t}^{\infty}\left(B_{s, p}^{3\left(1-\frac{1}{p}\right)}\right)}+\|\overline{\mathbf{d}}\|_{L_{t}^{p}\left(W^{3, s}\right)}\right),
\end{aligned}
$$

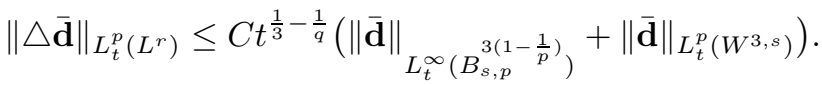

Define

$$
\begin{aligned}
X(t):= & \|\bar{\rho}\|_{L_{t}^{\infty}\left(L^{r}\right)}+\|\overline{\mathbf{u}}\|_{L_{t}^{\infty}\left(D_{A_{s}}^{1-\frac{1}{p}, p}\right)}+\|\overline{\mathbf{u}}\|_{L_{t}^{p}\left(W^{2, s}\right)}+\left\|\partial_{t} \overline{\mathbf{u}}\right\|_{L_{t}^{p}\left(L^{s}\right)} \\
& +\|\nabla \bar{P}\|_{L_{t}^{p}\left(L^{s}\right)}+\|\overline{\mathbf{d}}\|_{L_{t}^{\infty}\left(B_{s, p}^{3\left(1-\frac{1}{p}\right)}\right)}+\|\overline{\mathbf{d}}\|_{\mathcal{W}_{s, p}(0, t)} .
\end{aligned}
$$

Thus, combining (4.31)-4.33) and $B_{s, p}^{3\left(1-\frac{1}{p}\right)} \hookrightarrow W^{1, s}(\Omega) \hookrightarrow L^{r}(\Omega)$, we have

$$
\begin{aligned}
& X(t) \leq C\left\{t^{1-\frac{1}{p}}\left\|\rho_{2}\right\|_{L_{t}^{\infty}\left(W^{1, r}\right)}\left(1+\left\|\partial_{t} \mathbf{u}_{2}\right\|_{L_{t}^{p}\left(L^{q}\right)}+\left\|\mathbf{u}_{1}\right\|_{L_{t}^{\infty}\left(D_{A_{q}}^{1-\frac{1}{p}, p}\right)}\left\|\mathbf{u}_{1}\right\|_{L_{t}^{p}\left(W^{2, q}\right)}\right)\right. \\
& +t^{\frac{1}{2}-\frac{3}{2 q}}\left\|\mathbf{u}_{2}\right\|_{L_{t}^{\infty}\left(D_{A_{q}}^{1-\frac{1}{p}, p}\right)}+t^{\frac{2}{3}-\frac{1}{q}}\left\|\mathbf{u}_{1}\right\|_{L_{t}^{\infty}\left(D_{A_{q}}^{1-\frac{1}{p}, p}\right)}+\left\|\mathbf{u}_{1}\right\|_{L_{t}^{p}\left(W^{2, q}\right)} \\
& +t^{\frac{1}{3}-\frac{1}{q}}\left(\left\|\mathbf{d}_{1}-\overline{\mathbf{d}}\right\|_{L_{t}^{\infty}\left(B_{q, p}^{3\left(1-\frac{1}{p}\right)}\right)}+\left\|\mathbf{d}_{2}-\overline{\mathbf{d}}\right\|_{L_{t}^{\infty}\left(B_{q, p}^{3\left(1-\frac{1}{p}\right)}\right)}\right)+\left\|\mathbf{d}_{2}-\overline{\mathbf{d}}\right\|_{L_{t}^{p}\left(W^{2, q}\right)} \\
& +\left\|\mathbf{d}_{1}-\overline{\mathbf{d}}\right\|_{L_{t}^{p}\left(W^{3, q}\right)}+\left\|\mathbf{d}_{2}-\overline{\mathbf{d}}\right\|_{L_{t}^{p}\left(W^{3, q}\right)}+\left\|\mathbf{d}_{1}-\overline{\mathbf{d}}\right\|_{L_{t}^{\infty}\left(B_{q, p}^{3\left(1-\frac{1}{p}\right)}\right)}\left\|\mathbf{d}_{1}-\overline{\mathbf{d}}\right\|_{L_{t}^{p}\left(W^{2, q}\right)} \\
& \left.+\left(\left\|\mathbf{d}_{2}-\overline{\mathbf{d}}\right\|_{L_{t}^{\infty}\left(B_{q, p}^{3\left(1-\frac{1}{p}\right)}\right)}+1\right)\left(\left\|\mathbf{d}_{1}-\overline{\mathbf{d}}\right\|_{L_{t}^{p}\left(W^{2, q}\right)}+\left\|\mathbf{d}_{2}-\overline{\mathbf{d}}\right\|_{L_{t}^{p}\left(W^{2, q}\right)}\right)\right\} X(t) .
\end{aligned}
$$

Now, choosing $\eta$ so small that the term between brackets is less than $\frac{1}{2}$ for $t=\eta$ enables us to conclude that $X \equiv 0$ on $[0, \eta]$. As the constant $C$ does not depend on $\eta$, a standard induction argument yields the uniqueness on $\left[0, T_{0}\right]$.

Finally, as $\rho$ satisfies a transport equation with data in $W^{1, r}(\Omega)$, $\mathbf{u}$ satisfies

$$
\rho \partial_{t} \mathbf{u}-\triangle \mathbf{u}+\nabla P \in\left(L^{p}\left(0, t ; L^{q}(\Omega)\right)\right)^{3},
$$

and $\mathbf{d}$ satisfies

$$
\partial_{t} \mathbf{d}-\triangle \mathbf{d} \in\left(L^{p}\left(0, t ; L^{q}(\Omega)\right)\right)^{3},
$$

then Proposition 3.1 and Theorems 3.1$] 3.2$ insure that $\rho \in C\left(\left[0, T_{0}\right] ; W^{1, r}(\Omega)\right)$ (if $r \neq \infty), \mathbf{u} \in C\left(\left[0, T_{0}\right] ; D_{A_{q}}^{1-\frac{1}{p}, p}\right)$ and $\mathbf{d}-\overline{\mathbf{d}} \in C\left(\left[0, T_{0}\right] ; B_{q, p}^{3\left(1-\frac{1}{p}\right)}\right)$.

Remark 4.1. Following the argument of uniqueness and continuity, we can also easily prove that if $\left(\rho_{1}, \mathbf{u}_{1}, P_{1}, \mathbf{d}_{1}\right)$ and $\left(\rho_{2}, \mathbf{u}_{2}, P_{2}, \mathbf{d}_{2}\right)$ are solutions to (1.1)-(1.3) with different initial data $\left(\rho_{0}^{1}, \mathbf{u}_{0}^{1}, \mathbf{d}_{0}^{1}\right)$ and $\left(\rho_{0}^{2}, \mathbf{u}_{0}^{2}, \mathbf{d}_{0}^{2}\right)$, then the following estimate holds true on $\left[0, T_{0}\right]$ :

$$
\begin{aligned}
& \|\bar{\rho}(t)\|_{L^{r}}+\|\overline{\mathbf{u}}(t)\|_{D_{A_{s}^{1-\frac{1}{p}, p}}+\|\overline{\mathbf{u}}\|_{L_{t}^{p}\left(W^{2, s}\right)}+\left\|\partial_{t} \overline{\mathbf{u}}\right\|_{L_{t}^{p}\left(L^{s}\right)}} \\
& +\|\bar{P}\|_{L_{t}^{p}\left(W^{1, s}\right)}+\|\overline{\mathbf{d}}(t)\|_{B_{s, p}^{3\left(1-\frac{1}{p}\right)}}+\|\overline{\mathbf{d}}\|_{\mathcal{W}_{s, p}(0, t)} \\
& \leq C\left(\left\|\bar{\rho}_{0}\right\|_{L^{r}}+\left\|\overline{\mathbf{u}}_{0}\right\|_{D_{A_{s}}^{1-\frac{1}{p}, p}}+\left\|\overline{\mathbf{d}}_{0}\right\|_{B_{s, p}^{3\left(1-\frac{1}{p}\right)}}\right),
\end{aligned}
$$


where $\bar{\rho}_{0}:=\rho_{0}^{1}-\rho_{0}^{2}, \overline{\mathbf{u}}_{0}:=\mathbf{u}_{0}^{1}-\mathbf{u}_{0}^{2}, \overline{\mathbf{d}}_{0}:=\mathbf{d}_{0}^{1}-\mathbf{d}_{0}^{2}$. Combining with Theorem 2.1, we conclude that for small enough $T$, the map $\left(\rho_{0}, \mathbf{u}_{0}, \mathbf{d}_{0}-\overline{\mathbf{d}}\right) \rightarrow(\rho, \mathbf{u}, P, \mathbf{d}-\overline{\mathbf{d}})$ is Lipschitz continuous from bounded sets of $W^{1, r} \times D_{A_{q}}^{1-\frac{1}{p}, p} \times B_{q, p}^{3\left(1-\frac{1}{p}\right)}$ to

$$
\begin{array}{r}
C\left([0, T] ; L^{r}(\Omega)\right) \times\left(C\left([0, T] ; D_{A_{s}}^{1-\frac{1}{p}, p}\right) \cap\left(W^{1, p}\left(0, T ; L^{s}(\Omega)\right)\right)^{3} \cap\left(L^{p}\left(0, T ; W^{2, s}(\Omega)\right)\right)^{3}\right) \\
\times L^{p}\left(0, T ; W^{1, s}(\Omega)\right) \times\left(C\left([0, T] ; B_{s, p}^{3\left(1-\frac{1}{p}\right)}\right) \cap\left(W^{1, p}\left(0, T ; L^{s}(\Omega)\right)\right)^{3}\right. \\
\left.\cap\left(L^{p}\left(0, T ; W^{3, s}(\Omega)\right)\right)^{3}\right) .
\end{array}
$$

\section{Global existence}

In this section, we prove that if the initial data of velocity and orientation field is sufficiently 'small' in appropriate norms, with the help of uniform estimates, the local strong solution $(\rho, \mathbf{u}, P, \mathbf{d})$ of (1.1)-(1.3) established in the previous section is indeed global in time.

\subsection{Estimates for $\|\mathbf{u}\|_{L^{2}}$ and $\|\mathbf{d}\|_{L^{2}}$.}

Lemma 5.1. Let $\Omega, p, q, r$ be as in Theorem 2.1 and let $(\rho, \mathbf{u}, P, \mathbf{d})$ with $(\rho, \mathbf{u}, P$, $\mathbf{d}-\overline{\mathbf{d}}) \in M_{T_{0}}^{p, q, r}$ be a solution to (1.1)-(1.3) on $\Omega \times\left[0, T_{0}\right]$. Then the following inequality holds true for all $t \in\left[0, T_{0}\right]$ :

$$
\begin{aligned}
& \|(\sqrt{\rho} \mathbf{u})(t)\|_{L^{2}}^{2}+\|\nabla \mathbf{d}(t)\|_{L^{2}}^{2} \\
& \leq e^{-\frac{2 \lambda_{1}}{\hat{\rho}} t}\left(\left\|\sqrt{\rho_{0}} \mathbf{u}_{0}\right\|_{L^{2}}^{2}+\left\|\nabla \mathbf{d}_{0}\right\|_{L^{2}}^{2}\right)\left(1+\frac{2 \lambda_{1}}{\hat{\rho}} t e^{\frac{2 \lambda_{1}}{\hat{\rho}} t}\right),
\end{aligned}
$$

where $\lambda_{1}$ stands for the first eigenvalue of the Dirichlet-Laplace operator in $\Omega$.

Proof. Due to the inhomogeneous incompressible character the flows we are dealing with, the natural framework in which we shall work is that of the solenoidal vector field of $L^{2}(\Omega)^{3}$. Note that

$$
\begin{gathered}
\mathbf{u} \in C\left(\left[0, T_{0}\right] ; D_{A_{q}}^{1-\frac{1}{p}, p}\right) \cap\left(L^{p}\left(0, T_{0} ; W^{2, q}(\Omega) \cap W_{0}^{1, q}(\Omega)\right)\right)^{3}, \\
\mathbf{d}-\overline{\mathbf{d}} \in C\left(\left[0, T_{0}\right] ; B_{q, p}^{3\left(1-\frac{1}{p}\right)}\right) \cap\left(L^{p}\left(0, T_{0} ; W^{3, q}(\Omega)\right)\right)^{3} .
\end{gathered}
$$

And, since

$$
D_{A_{q}}^{1-\frac{1}{p}, p} \hookrightarrow B_{q, p}^{2\left(1-\frac{1}{p}\right)} \cap X^{q}
$$

(see Proposition 2.5 in [8]) where

$$
X^{q}=\left\{\mathbf{z} \in L^{q}(\Omega)^{3} \mid \nabla \cdot \mathbf{z}=0 \text { in } \Omega \text { and } \mathbf{z} \cdot \mathbf{n}=0 \text { on } \partial \Omega\right\},
$$

then when $1<p<2$, by the standard interpolation inequality

$$
L^{\infty}\left(0, T_{0} ; L^{q}(\Omega)\right) \cap L^{p}\left(0, T_{0} ; W^{2, q}(\Omega)\right) \subset L^{2}\left(0, T_{0} ; H^{1+\alpha}(\Omega)\right),
$$

where

we have

$$
\frac{1}{2}=\frac{\theta}{p}, \quad \frac{1}{2}-\frac{1+\alpha}{3}=(1-\theta) \frac{1}{q}+\theta\left(\frac{1}{q}-\frac{2}{3}\right),
$$

$$
\begin{gathered}
\mathbf{u} \in\left(C\left(\left[0, T_{0}\right] ; H^{\alpha}(\Omega)\right) \cap L^{2}\left(0, T_{0} ; H^{1+\alpha}(\Omega)\right)\right)^{3}, \\
\mathbf{d}-\overline{\mathbf{d}} \in\left(C\left(\left[0, T_{0}\right] ; H^{1+\alpha}(\Omega)\right) \cap L^{2}\left(0, T_{0} ; H^{2+\alpha}(\Omega)\right)\right)^{3} .
\end{gathered}
$$


When $2 \leq p<\infty$, (5.1)-(5.2) hold obviously due to $W^{2, q}(\Omega) \hookrightarrow H^{2}(\Omega)$ as $q>3$.

Now, $\rho$ is continuous in $(\mathbf{x}, t), \mathbf{u} \in\left(C\left(\left[0, T_{0}\right] ; H^{\alpha}(\Omega)\right) \cap L^{2}\left(0, T_{0} ; H^{1+\alpha}(\Omega)\right)\right)^{3}$ and $\mathbf{d}-\overline{\mathbf{d}} \in\left(C\left(\left[0, T_{0}\right] ; H^{1+\alpha}(\Omega)\right) \cap L^{2}\left(0, T_{0} ; H^{2+\alpha}(\Omega)\right)\right)^{3}$. This enables us to justify the following computations.

Taking the $L^{2}$ scalar product in $(1.1 \mathrm{~b})$ with $\mathbf{u}$ and performing integration by parts, using the continuity equation (1.1a), we obtain

$$
\begin{aligned}
& \frac{1}{2} \frac{d}{d t} \int_{\Omega} \rho|\mathbf{u}|^{2} d \mathbf{x}+\int_{\Omega}|\nabla \mathbf{u}|^{2} d \mathbf{x} \\
& =-\frac{1}{2} \int_{\Omega}|\mathbf{u}|^{2} \nabla \cdot(\rho \mathbf{u}) d \mathbf{x}-\int_{\Omega} \rho \mathbf{u} \cdot \nabla \mathbf{u} \cdot \mathbf{u} d \mathbf{x}-\int_{\Omega} \mathbf{u} \cdot\left((\nabla \mathbf{d})^{\top} \triangle \mathbf{d}\right) d \mathbf{x} \\
& =-\int_{\Omega} \mathbf{u} \cdot\left((\nabla \mathbf{d})^{\top} \triangle \mathbf{d}\right) d \mathbf{x} .
\end{aligned}
$$

Here we have used the facts

$$
\nabla \cdot(\nabla \mathbf{d} \odot \nabla \mathbf{d})=\nabla\left(\frac{|\nabla \mathbf{d}|^{2}}{2}\right)+(\nabla \mathbf{d})^{\top} \triangle \mathbf{d}
$$

and $\nabla \cdot \mathbf{u}=0$ in $\Omega, \mathbf{u}=0$ on $\partial \Omega$, as well as

$$
\int_{\Omega} \nabla P \cdot \mathbf{u} d \mathbf{x}=\int_{\Omega} \nabla\left(\frac{|\nabla \mathbf{d}|^{2}}{2}\right) \cdot \mathbf{u} d \mathbf{x}=0 .
$$

Multiplying (1.1c) by $-\left(\triangle \mathbf{d}+|\nabla \mathbf{d}|^{2} \mathbf{d}\right)$ and integrating over $\Omega$, we obtain

$$
-\int_{\Omega} \partial_{t} \mathbf{d} \cdot \triangle \mathbf{d} d \mathbf{x}-\int_{\Omega}(\mathbf{u} \cdot \nabla \mathbf{d}) \cdot \triangle \mathbf{d} d \mathbf{x}=-\left.\left.\int_{\Omega}|\triangle \mathbf{d}+| \nabla \mathbf{d}\right|^{2} \mathbf{d}\right|^{2} d \mathbf{x} .
$$

Here we have used the fact that $|\mathbf{d}|=1$ to get

$$
\left(\partial_{t} \mathbf{d}+\mathbf{u} \cdot \nabla \mathbf{d}\right) \cdot|\nabla \mathbf{d}|^{2} \mathbf{d}=\frac{1}{2}\left(|\nabla \mathbf{d}|^{2} \partial_{t}\left(|\mathbf{d}|^{2}\right)+\mathbf{u} \cdot \nabla|\mathbf{d}|^{2}|\nabla \mathbf{d}|^{2}\right)=0 .
$$

Since $\partial_{\nu} \mathbf{d}=0$ on $\partial \Omega$, integrating by parts, we have

$$
\int_{\Omega} \partial_{t} \mathbf{d} \cdot \triangle \mathbf{d} d \mathbf{x}=-\frac{1}{2} \frac{d}{d t} \int_{\Omega}|\nabla \mathbf{d}|^{2} d \mathbf{x}
$$

Hence we obtain

$$
\frac{1}{2} \frac{d}{d t} \int_{\Omega}|\nabla \mathbf{d}|^{2} d \mathbf{x}+\left.\left.\int_{\Omega}|\Delta \mathbf{d}+| \nabla \mathbf{d}\right|^{2} \mathbf{d}\right|^{2} d \mathbf{x}=\int_{\Omega}(\mathbf{u} \cdot \nabla \mathbf{d}) \cdot \Delta \mathbf{d} d \mathbf{x} .
$$

By adding (5.3) and (5.4), we eventually get the identity

$$
\frac{1}{2} \frac{d}{d t} \int_{\Omega}\left(\rho|\mathbf{u}|^{2}+|\nabla \mathbf{d}|^{2}\right) d \mathbf{x}+\int_{\Omega}\left(|\nabla \mathbf{u}|^{2}+\left.\left.|\triangle \mathbf{d}+| \nabla \mathbf{d}\right|^{2} \mathbf{d}\right|^{2}\right) d \mathbf{x}=0 .
$$

Since $\nabla \mathbf{d} \in L^{2}\left(0, T_{0} ; H^{1}(\Omega)\right)$ and $|\mathbf{d}|=1$, we have

$$
\triangle \mathbf{d} \cdot \mathbf{d}+|\nabla \mathbf{d}|^{2}=0
$$

and then

$$
\left.\left.\int_{\Omega}|\triangle \mathbf{d}+| \nabla \mathbf{d}\right|^{2} \mathbf{d}\right|^{2} d \mathbf{x}=\int_{\Omega}|\triangle \mathbf{d} \times \mathbf{d}|^{2} d \mathbf{x}=\int_{\Omega}\left|\partial_{t} \mathbf{d}\right|^{2} d \mathbf{x} .
$$

The last equality comes from $\Delta \mathbf{d}=\partial_{t} \mathbf{d}+\mathbf{u} \cdot \nabla \mathbf{d}-|\nabla \mathbf{d}|^{2} \mathbf{d}$ and the fact that $|\mathbf{d}|=1$.

Now, by virtue of the Poincaré inequality

$$
\|\nabla \mathbf{u}\|_{L^{2}}^{2} \geq \lambda_{1}\|\mathbf{u}\|_{L^{2}}^{2}
$$


and the inequality

we get from (5.5) that

$$
\left\|\partial_{t} \mathbf{d}\right\|_{L^{2}} \geq \frac{d}{d t}\|\mathbf{d}(t)\|_{L^{2}}=0
$$

$$
\frac{1}{2} \frac{d}{d t}\left(\|\sqrt{\rho} \mathbf{u}\|_{L^{2}}^{2}+\|\nabla \mathbf{d}\|_{L^{2}}^{2}\right)+\frac{\lambda_{1}}{\hat{\rho}}\|\sqrt{\rho} \mathbf{u}\|_{L^{2}}^{2} \leq 0,
$$

i.e.

$$
\frac{d}{d t}\left(e^{\frac{2 \lambda_{1}}{\hat{\rho}} t}\|\sqrt{\rho} \mathbf{u}\|_{L^{2}}^{2}\right)+e^{\frac{2 \lambda_{1}}{\hat{\rho}} t} \frac{d}{d t}\|\nabla \mathbf{d}\|_{L^{2}}^{2} \leq 0 .
$$

Integrating (5.7) from 0 to $t$, we obtain

$$
\begin{aligned}
& e^{\frac{2 \lambda_{1}}{\hat{\rho}} t}\left(\|(\sqrt{\rho} \mathbf{u})(t)\|_{L^{2}}^{2}+\|\nabla \mathbf{d}(t)\|_{L^{2}}^{2}\right) \\
& \leq\left\|\sqrt{\rho_{0}} \mathbf{u}_{0}\right\|_{L^{2}}^{2}+\left\|\nabla \mathbf{d}_{0}\right\|_{L^{2}}^{2}+\frac{2 \lambda_{1}}{\hat{\rho}} \int_{0}^{t} e^{\frac{2 \lambda_{1}}{\hat{\rho}} \tau}\|\nabla \mathbf{d}(\tau)\|_{L^{2}}^{2} d \tau .
\end{aligned}
$$

It follows from Grönwall's inequality that

$$
e^{\frac{2 \lambda_{1}}{\hat{\rho}} t}\|\nabla \mathbf{d}\|_{L^{2}}^{2} \leq\left(\left\|\sqrt{\rho_{0}} \mathbf{u}_{0}\right\|_{L^{2}}^{2}+\left\|\nabla \mathbf{d}_{0}\right\|_{L^{2}}^{2}\right)\left(1+\frac{2 \lambda_{1}}{\hat{\rho}} t e^{\frac{2 \lambda_{1}}{\hat{\rho}} t}\right),
$$

and furthermore,

$e^{\frac{2 \lambda_{1}}{\hat{\rho}} t}\left(\|\sqrt{\rho} \mathbf{u}\|_{L^{2}}^{2}+\|\nabla \mathbf{d}\|_{L^{2}}^{2}\right) \leq\left(\left\|\sqrt{\rho_{0}} \mathbf{u}_{0}\right\|_{L^{2}}^{2}+\left\|\nabla \mathbf{d}_{0}\right\|_{L^{2}}^{2}\right)\left(2+\frac{2 \lambda_{1}}{\hat{\rho}} t e^{\frac{2 \lambda_{1}}{\hat{\rho}} t}-e^{\frac{2 \lambda_{1}}{\hat{\rho}} t}+\frac{2 \lambda_{1}}{\hat{\rho}} t\right)$.

Usually (5.5) is called the basic energy law governing the system (1.1)-(1.3). It reflects the energy dissipation property of the flow of liquid crystals.

5.2. A more explicit lower bound for the existence time. We denote by $T^{*}$ the maximal existence time for $(\rho, \mathbf{u}, P, \mathbf{d})$ which means $(\rho, \mathbf{u}, P, \mathbf{d})$ cannot be continued beyond $T^{*}$ into a strong solution of (1.1)-(1.3). Let us first state a continuation criterion:

Lemma 5.2. Let $\rho_{0}, \mathbf{u}_{0}, \mathbf{d}_{0}$ be as in Theorem 2.1 and assume that system (1.1) with the initial-boundary conditions (1.2)-(1.3) has a strong solution on a finite time interval $\left[0, T^{*}\right)$ with

$$
\begin{gathered}
\rho \in L^{\infty}\left(0, T^{*} ; W^{1, r}(\Omega)\right), \inf _{t<T^{*}, \mathbf{x} \in \Omega} \rho(\mathbf{x}, t)>0 \\
\mathbf{u} \in L^{\infty}\left(0, T^{*} ; D_{A_{q}}^{1-\frac{1}{p}, p}\right) \quad \text { and } \quad \mathbf{d}-\overline{\mathbf{d}} \in L^{\infty}\left(0, T^{*} ; B_{q, p}^{3\left(1-\frac{1}{p}\right)}\right) .
\end{gathered}
$$

Then $(\rho, \mathbf{u}, P, \mathbf{d})$ can be continued beyond $T^{*}$ into a strong solution of (1.1)-(1.3).

Proof. Indeed, a positive lower bound $\check{T}$ for the existence time has already been obtained in the proof of Theorem 2.1 (see (4.21), (4.22) and (4.28) when $\left(\rho_{0}, \mathbf{u}_{0}\right.$, $\left.\mathbf{d}_{0}-\overline{\mathbf{d}}\right)$ remains in a bounded set of

$$
W^{1, r} \times D_{A_{q}}^{1-\frac{1}{p}, p} \times B_{q, p}^{3\left(1-\frac{1}{p}\right)}
$$

with, in addition, $\inf _{\mathbf{x} \in \Omega} \rho_{0}(\mathbf{x}) \geq \check{\rho}$ for a fixed $\check{\rho}>0$. Hence system (1.1) with initial density $\rho\left(T^{*}-\frac{\check{T}}{2}\right)$, initial velocity $\mathbf{u}\left(T^{*}-\frac{\check{T}}{2}\right)$ and initial orientation field $\mathbf{d}\left(T^{*}-\frac{\check{T}}{2}\right)$ has a unique strong solution on $[0, \check{T}]$ which provides a continuation of the strong solution beyond $T^{*}$. 
Combining Lemma 5.1 and Lemma 5.2 enables us to get the following result:

Proposition 5.1. Let $\rho_{0}, \mathbf{u}_{0}, \mathbf{d}_{0}$ be as in Theorem 2.1 and let $(\rho, \mathbf{u}, P, \mathbf{d})$ denote the corresponding strong solution of (1.1)-(1.3). Then there exists some constant $C$ depending on $p, q, r, \mu, \lambda, \gamma, \Omega$ and $\check{\rho}$ such that the maximal existence time $T^{*}$ for $(\rho, \mathbf{u}, P, \mathbf{d})$ satisfies

$$
T^{*} \geq \frac{C}{\left(1+\left\|\rho_{0}\right\|_{W^{1, r}}\right)^{\kappa}\left(U^{0}\right)^{\iota}}
$$

for some positive exponents $\kappa$ and $\iota$ depending only on the regularity parameters.

Proof. Fix a $\tilde{T}<T^{*}$. We aim to prove that if $\tilde{T} \leq C\left(1+\left\|\rho_{0}\right\|_{W^{1, r}}\right)^{-\kappa}\left(U^{0}\right)^{-\iota}$ for a convenient choice of $C, \kappa$ and $\iota$, then $(\rho, \mathbf{u}, P, \mathbf{d}-\overline{\overline{\mathbf{d}}})$ may be bounded in $M_{\tilde{T}}^{p, q, r}$ by a function depending only on the data. Then Lemma 5.2 will entail Proposition 5.1

Define

$$
\begin{aligned}
G(t):= & \|\mathbf{u}\|_{L_{t}^{\infty}\left(D_{A_{q}}^{1-\frac{1}{p}, p}\right)}+\|\mathbf{u}\|_{L_{t}^{p}\left(W^{2, q}\right)}+\left\|\partial_{t} \mathbf{u}\right\|_{L_{t}^{p}\left(L^{q}\right)} \\
& +\|\nabla P\|_{L_{t}^{p}\left(L^{q}\right)}+\|\mathbf{d}-\overline{\mathbf{d}}\|_{L_{t}^{\infty}\left(B_{q, p}^{3\left(1-\frac{1}{p}\right)}\right)}+\|\mathbf{d}-\overline{\mathbf{d}}\|_{\mathcal{W}_{q, p}(0, t)} .
\end{aligned}
$$

According to Theorems 3.13.2 and (4.7), we have

$$
\begin{aligned}
G(t) \leq C( & \mathcal{B}_{\rho}^{2}(t)\left(\left\|\mathbf{u}_{0}\right\|_{D_{A_{q}}^{1-\frac{1}{p}, p}}+\|\mathbf{u} \cdot \nabla \mathbf{u}\|_{L_{t}^{p}\left(L^{q}\right)}+\left\|\nabla \cdot\left((\nabla \mathbf{d})^{\top} \nabla \mathbf{d}\right)\right\|_{L_{t}^{p}\left(L^{q}\right)}\right) \\
& \left.+\mathcal{C}_{\rho}(t)\|\mathbf{u}\|_{L_{t}^{p}\left(L^{q}\right)}+\left\|\mathbf{d}_{0}\right\|_{B_{q, p}^{3\left(1-\frac{1}{p}\right)}}+\left\|-\mathbf{u} \cdot \nabla \mathbf{d}+|\nabla \mathbf{d}|^{2} \mathbf{d}\right\|_{L_{t}^{p}\left(L^{q}\right)}\right)
\end{aligned}
$$

where $C=C(p, q, r, \Omega, \check{\rho}, \hat{\rho})$.

Combining the Gagliardo-Nirenberg-Sobolev inequality and Young's inequality yields, for all $\varepsilon>0$,

$$
\|\mathbf{u}\|_{L^{q}} \leq C\left(\varepsilon\|\mathbf{u}\|_{W^{2, q}}+\varepsilon^{1-\frac{1}{\theta}}\|\mathbf{u}\|_{L^{2}}\right) \quad \text { with } \quad \theta=\frac{4 q}{7 q-6} .
$$

We note that Lemma 5.1 insures

$$
\|\mathbf{u}\|_{L_{t}^{\infty}\left(L^{2}\right)} \leq C\left(1+t^{\frac{1}{2}}\right) U^{0}
$$

then employing Hölder's inequality, we have

$$
\|\mathbf{u}\|_{L_{t}^{p}\left(L^{2}\right)} \leq t^{\frac{1}{p}}\|\mathbf{u}\|_{L_{t}^{\infty}\left(L^{2}\right)} \leq C t^{\frac{1}{p}}\left(1+t^{\frac{1}{2}}\right) U^{0} .
$$

Moreover, we get

$$
\|\mathbf{u} \cdot \nabla \mathbf{u}\|_{L_{t}^{p}\left(L^{q}\right)} \leq\|\mathbf{u}\|_{L_{t}^{\infty}\left(L^{q}\right)}\|\nabla \mathbf{u}\|_{L_{t}^{p}\left(L^{\infty}\right)} \leq C t^{\frac{1}{2}-\frac{3}{2 q}} G^{2}(t),
$$

$\left\|\nabla \cdot\left((\nabla \mathbf{d})^{\top} \nabla \mathbf{d}\right)\right\|_{L_{t}^{p}\left(L^{q}\right)} \leq C\|\nabla(\mathbf{d}-\overline{\mathbf{d}})\|_{L_{t}^{\infty}\left(L^{q}\right)}\|\triangle(\mathbf{d}-\overline{\mathbf{d}})\|_{L_{t}^{p}\left(L^{\infty}\right)} \leq C t^{\frac{1}{3}-\frac{1}{q}} G^{2}(t)$,

$$
\begin{aligned}
& \left\|-\mathbf{u} \cdot \nabla \mathbf{d}+|\nabla \mathbf{d}|^{2} \mathbf{d}\right\|_{L_{t}^{p}\left(L^{q}\right)} \\
& \leq\|\mathbf{u}\|_{L_{t}^{\infty}\left(L^{q}\right)}\|\nabla(\mathbf{d}-\overline{\mathbf{d}})\|_{L_{t}^{p}\left(L^{\infty}\right)}+\|\nabla(\mathbf{d}-\overline{\mathbf{d}})\|_{L_{t}^{\infty}\left(L^{q}\right)}\|\nabla(\mathbf{d}-\overline{\mathbf{d}})\|_{L_{t}^{p}\left(L^{\infty}\right)} \\
& \leq C t^{\frac{1}{2}-\frac{3}{2 q}} G^{2}(t) .
\end{aligned}
$$


Here we have used the fact that $|\mathbf{d}|=1$. Hence plugging (5.9)-(5.13) into (5.8) while taking $\varepsilon=\epsilon \mathcal{C}_{\rho}^{-1}(t)$ with $\epsilon$ suitably small, we get

$$
\begin{aligned}
G(t) \leq C\left(\mathcal { B } _ { \rho } ^ { 2 } ( t ) \left(U^{0}+\left(t^{\frac{1}{2}-\frac{3}{2 q}}\right.\right.\right. & \left.\left.+t^{\frac{1}{3}-\frac{1}{q}}\right) G^{2}(t)\right) \\
& \left.+\mathcal{C}_{\rho}^{\frac{1}{\theta}}(t) t^{\frac{1}{p}}\left(1+t^{\frac{1}{2}}\right) U^{0}+U^{0}+t^{\frac{1}{2}-\frac{3}{2 q}} G^{2}(t)\right)
\end{aligned}
$$

On the other hand, using the same argument as for $\rho^{k+1}$ in Subsection 4.2, we obtain

$$
\begin{aligned}
\|\nabla \rho\|_{L_{t}^{\infty}\left(L^{r}\right)} \leq\left\|\rho_{0}\right\|_{W^{1, r}} e^{C t^{\frac{3}{2}-\frac{1}{p}-\frac{3}{2 q}} G(t)} \\
\|\rho\|_{C_{t}^{\beta}\left(L^{\infty}\right)} \leq C\left(\|\rho\|_{L_{t}^{\infty}\left(W^{1, r}\right)}+\left\|\partial_{t} \rho\right\|_{L_{t}^{\infty}\left(L^{s}\right)}\right) \\
\leq C\left(\|\rho\|_{L_{t}^{\infty}\left(W^{1, r}\right)}+\|\mathbf{u}\|_{L_{t}^{\infty}\left(L^{q}\right)}\|\nabla \rho\|_{L_{t}^{\infty}\left(L^{r}\right)}\right) \\
\leq C\|\nabla \rho\|_{L_{t}^{\infty}\left(L^{r}\right)}(1+G(t)) .
\end{aligned}
$$

Then, according to the definitions of $\mathcal{B}_{\rho}(t)$ and $\mathcal{C}_{\rho}(t)$ in Theorem 3.7 in $[8$, using (5.15) and (5.16), we eventually get

$$
\mathcal{B}_{\rho}(t) \leq C e^{C t^{\frac{3}{2}-\frac{1}{p}-\frac{3}{2 q}} G(t)}\left(1+\left\|\rho_{0}\right\|_{W^{1, r}}\right)^{\frac{r}{r-3}}
$$

$\mathcal{C}_{\rho}(t) \leq C e^{C t^{\frac{3}{2}-\frac{1}{p}-\frac{3}{2 q}}} G(t)\left(\left(1+\left\|\rho_{0}\right\|_{W^{1, r}}\right)^{\gamma_{1}}+\left(1+\left\|\rho_{0}\right\|_{W^{1, r}}\right)^{\gamma_{2}}\left\|\rho_{0}\right\|_{W^{1, r}}^{\frac{1}{\beta}}(1+G(t))^{\frac{1}{\beta}}\right)$,

where $\gamma_{1}$ and $\gamma_{2}$ depend only on $p, q, r$ and $\beta$.

Plugging (5.17)-(5.18) into (5.14), for some positive exponents $\delta_{1}$ and $\delta_{2}$, we have

$$
\begin{aligned}
G(t) \leq & C e^{C t^{\frac{3}{2}-\frac{1}{p}-\frac{3}{2 q}} G(t)}\left(1+\left\|\rho_{0}\right\|_{W^{1, r}}\right)^{\delta_{1}}\left(U^{0}\left(1+t^{\frac{1}{p}}\left(1+t^{\frac{1}{2}}\right)(1+G(t))^{\delta_{2}}\right)\right. \\
& \left.+\left(t^{\frac{1}{2}-\frac{3}{2 q}}+t^{\frac{1}{3}-\frac{1}{q}}\right) G^{2}(t)\right) .
\end{aligned}
$$

Assume that $\tilde{T}$ has been chosen such that

$$
G(\tilde{T}) \leq 8 C\left(1+\left\|\rho_{0}\right\|_{W^{1, r}}\right)^{\delta_{1}} U^{0} .
$$

This is possible because of the continuity of the function $t \mapsto G(t)$. Noticing that $G(t)$ is increasing in $t$, a standard induction argument shows (5.19) is satisfied at time $t \leq \tilde{T}$ with a strict inequality whenever the following three inequalities are satisfied:

$$
\begin{gathered}
8 C^{2}\left(1+\left\|\rho_{0}\right\|_{W^{1, r}}\right)^{\delta_{1}} U^{0} t^{\frac{3}{2}-\frac{1}{p}-\frac{3}{2 q}}<\ln 2, \\
\left(1+8 C\left(1+\left\|\rho_{0}\right\|_{W^{1, r}}\right)^{\delta_{1}} U^{0}\right)^{\delta_{2}} t^{\frac{1}{p}}\left(1+t^{\frac{1}{2}}\right) \leq 1, \\
64 C^{2}\left(1+\left\|\rho_{0}\right\|_{W^{1, r}}\right)^{2 \delta_{1}} U^{0}\left(t^{\frac{1}{2}-\frac{3}{2 q}}+t^{\frac{1}{3}-\frac{1}{q}}\right) \leq 2 .
\end{gathered}
$$

Hence Lemma 5.2 enables us to continue the solution $(\rho, \mathbf{u}, P, \mathbf{d})$ beyond $\tilde{T}$.

The proof of Proposition 5.1 is complete. 
5.3. The case of a 'small' initial velocity and orientation field. Proposition 5.1 insures that the existence time of a strong solution for (1.1)-(1.3) goes to infinity (for fixed initial density) when $\mathbf{u}_{0}$ (resp. $\mathbf{d}_{0}-\overline{\mathbf{d}}$ ) tends to $\mathbf{0}$ in $D_{A_{q}}^{1-\frac{1}{p}, p}$ (resp. $\left.B_{q, p}^{3\left(1-\frac{1}{p}\right)}\right)$. We now aim to state that the system has indeed a global strong solution if $\mathbf{u}_{0}$ and $\mathbf{d}_{0}$ are suitably small. This will give Theorem 2.2

Let $(\rho, \mathbf{u}, P, \mathbf{d})$ be the strong solution given by Theorem 2.1. For any $\zeta \geq 0$, define

$$
G_{0,2, \zeta}(t):=\left(\left\|\sqrt{\rho_{0}} \mathbf{u}_{0}\right\|_{L^{2}}+\left\|\nabla \mathbf{d}_{0}\right\|_{L^{2}}\right)\left(1+(2 \zeta t)^{\frac{1}{2}} e^{\zeta t}\right) \quad \text { and } \quad G_{0,2}:=G_{0,2,0}(t) .
$$

By Lemma 5.1, for $t<T^{*}$, we have

$$
\|\sqrt{\rho} \mathbf{u}\|_{L_{t}^{p}\left(L^{2}\right)} \leq C G_{0,2} t^{\frac{1}{p}}\left(1+\left(\frac{2 \lambda_{1}}{\hat{\rho}} t\right)^{\frac{1}{2}}\right)
$$

and

$$
\|(\sqrt{\rho} \mathbf{u})(t)\|_{L^{2}}+\|\nabla \mathbf{d}(t)\|_{L^{2}} \leq C e^{-\frac{\lambda_{1}}{\hat{\rho}} t} G_{0,2, \frac{\lambda_{1}}{\hat{\rho}}}(t) .
$$

Hence, starting from (5.8), using (5.9), (5.20) and the fact that

$$
\begin{aligned}
&\|\mathbf{u} \cdot \nabla \mathbf{u}\|_{L_{t}^{p}\left(L^{q}\right)} \leq\|\mathbf{u}\|_{L_{t}^{\infty}\left(L^{q}\right)}\|\nabla \mathbf{u}\|_{L_{t}^{p}\left(L^{\infty}\right)} \\
& \leq C\|\mathbf{u}\|_{L_{t}^{\infty}\left(D_{A_{q}}^{1-\frac{1}{p}, p}\right)}\|\mathbf{u}\|_{L_{t}^{p}\left(W^{2, q}\right)} \leq C G^{2}(t), \\
&\left\|\nabla \cdot\left((\nabla \mathbf{d})^{\top} \nabla \mathbf{d}\right)\right\|_{L_{t}^{p}\left(L^{q}\right)} \leq C\|\nabla \mathbf{d}\|_{L_{t}^{\infty}\left(L^{q}\right)}\|\Delta \mathbf{d}\|_{L_{t}^{p}\left(L^{\infty}\right)} \\
& \leq C\|\mathbf{d}-\overline{\mathbf{d}}\|_{L_{t}^{\infty}\left(B_{q, p}^{3\left(1-\frac{1}{p}\right)}\right)}\|\mathbf{d}-\overline{\mathbf{d}}\|_{L_{t}^{p}\left(W^{3, q}\right)} \leq C G^{2}(t), \\
&\left\|-\mathbf{u} \cdot \nabla \mathbf{d}+|\nabla \mathbf{d}|^{2} \mathbf{d}\right\|_{L_{t}^{p}\left(L^{q}\right)} \\
& \leq\|\mathbf{u}\|_{L_{t}^{\infty}\left(L^{q}\right)}\|\nabla \mathbf{d}\|_{L_{t}^{p}\left(L^{\infty}\right)}+\|\nabla \mathbf{d}\|_{L_{t}^{\infty}\left(L^{q}\right)}\|\nabla \mathbf{d}\|_{L_{t}^{p}\left(L^{\infty}\right)} \\
& \leq C\left(\|\mathbf{u}\|_{L_{t}^{\infty}\left(D_{A_{q}}^{1-\frac{1}{p}, p}\right)}\|\mathbf{d}-\overline{\mathbf{d}}\|_{L_{t}^{p}\left(W^{2, q}\right)}+\|\mathbf{d}-\overline{\mathbf{d}}\|_{L_{t}^{\infty}\left(B_{q, p}^{3\left(1-\frac{1}{p}\right)}\right)}\|\mathbf{d}-\overline{\mathbf{d}}\|_{L_{t}^{p}\left(W^{2, q}\right)}\right) \\
& \leq C G^{2}(t),
\end{aligned}
$$

we end up with

$$
G(t) \leq C\left(\left(1+\mathcal{B}_{\rho}^{2}(t)\right)\left(U^{0}+G^{2}(t)\right)+\mathcal{C}_{\rho}^{\frac{1}{\theta}}(t) G_{0,2} t^{\frac{1}{p}}\left(1+\left(\frac{2 \lambda_{1}}{\hat{\rho}} t\right)^{\frac{1}{2}}\right)\right) .
$$

Once again, the bounds for $\mathcal{B}_{\rho}(t)$ and $\mathcal{C}_{\rho}(t)$ will follow from (5.17) and (5.18). However, in contrast with the previous section, we are going to take advantage of Lemma 5.1 to avoid the appearance of the factor $t^{\frac{3}{2}-\frac{1}{p}-\frac{3}{2 q}}$. Indeed, since $\left(L^{2}(\Omega), W^{2, q}(\Omega)\right)_{\vartheta} \hookrightarrow W^{1, \infty}(\Omega)$ with $\vartheta=\frac{5 q}{7 q-6}$, it follows from Hölder's inequality that

$$
\begin{aligned}
\int_{0}^{t}\|\nabla \mathbf{u}(\tau)\|_{L^{\infty}} d \tau & \leq C \int_{0}^{t}\|\mathbf{u}(\tau)\|_{L^{2}}^{1-\vartheta}\|\mathbf{u}(\tau)\|_{W^{2, q}}^{\vartheta} d \tau \\
& \leq C \int_{0}^{t}\left(e^{-\frac{\lambda_{1}}{\hat{\rho}} \tau} G_{0,2, \frac{\lambda_{1}}{\hat{\rho}}}(\tau)\right)^{1-\vartheta}\|\mathbf{u}(\tau)\|_{W^{2, q}}^{\vartheta} d \tau \\
& \leq C G_{0,2, \frac{\lambda_{1}}{\hat{\rho}}}^{1-\vartheta}(t) G^{\vartheta}(t) .
\end{aligned}
$$


Now, bounding $\mathcal{B}_{\rho}(t)$ and $\mathcal{C}_{\rho}(t)$ may be done by mimicking the proof of Proposition 5.1, and we eventually conclude that

$$
G(t) \leq C e^{C G_{0,2, \frac{\lambda_{1}}{\hat{\rho}}}^{1-\vartheta}(t) G^{\vartheta}(t)}\left(1+\left\|\rho_{0}\right\|_{W^{1, r}}\right)^{\delta_{3}}\left(U^{0}\left(1+t^{\frac{1}{p}}\left(1+t^{\frac{1}{2}}\right)(1+G(t))^{\delta_{4}}\right)+G^{2}(t)\right)
$$

for some positive exponents $\delta_{3}$ and $\delta_{4}$ depending only on $p, q, r$.

Fix a positive $\bar{T}$ and assume that

$$
G(t) \leq 8 C\left(1+\left\|\rho_{0}\right\|_{W^{1, r}}\right)^{\delta_{3}} U^{0}, \quad t \in[0, \bar{T}] .
$$

If the data are so small that

$$
C G_{0,2, \frac{\lambda_{1}}{\hat{\rho}}}^{1-\vartheta}(\bar{T})\left(8 C\left(1+\left\|\rho_{0}\right\|_{W^{1, r}}\right)^{\delta_{3}} U^{0}\right)^{\vartheta} \leq \ln 2,
$$

then (5.21) implies

$$
G(t) \leq 2 C\left(1+\left\|\rho_{0}\right\|_{W^{1, r}}\right)^{\delta_{3}}\left(U^{0}\left(1+t^{\frac{1}{p}}\left(1+t^{\frac{1}{2}}\right)(1+G(t))^{\delta_{4}}\right)+G^{2}(t)\right) .
$$

Now, if in addition

$64 C^{2}\left(1+\left\|\rho_{0}\right\|_{W^{1, r}}\right)^{2 \delta_{3}} U^{0} \leq \frac{1}{2}$ and $\bar{T}^{\frac{1}{p}}\left(1+\bar{T}^{\frac{1}{2}}\right)\left(1+8 C\left(1+\left\|\rho_{0}\right\|_{W^{1, r}}\right)^{\delta_{3}} U^{0}\right)^{\delta_{4}} \leq \frac{3}{2}$,

then $(\underline{5.22})$ is satisfied with the constant $6 C$ instead of $8 C$. A standard bootstrap argument enables us to conclude the second part of Theorem 2.1.

\section{WeAK-STRong Uniqueness}

The purpose of this section is to show weak-strong uniqueness in Theorem 2.2 . To this end, we need to obtain first an energy estimate for the strong solution to system (1.1)-(1.3).

Lemma 6.1. Let $p, q, r$ satisfy the same conditions as Theorem 2.1 and $(\rho, \mathbf{u}, P, \mathbf{d})$ with $(\rho, \mathbf{u}, P, \mathbf{d}-\overline{\mathbf{d}}) \in M_{T_{0}}^{p, q, r}$ be the unique solution to (1.1)-(1.3) on $\Omega \times\left[0, T_{0}\right]$. Then for any $0<t \leq T_{0}$, we have

$$
\begin{aligned}
& \frac{1}{2} \int_{\Omega}\left(\rho(t)|\mathbf{u}(t)|^{2}+|\nabla \mathbf{d}(t)|^{2}\right) d \mathbf{x}+\int_{0}^{t} \int_{\Omega}\left(|\nabla \mathbf{u}|^{2}+\left.\left.|\triangle \mathbf{d}+| \nabla \mathbf{d}\right|^{2} \mathbf{d}\right|^{2}\right) d \mathbf{x} d \tau \\
& =\frac{1}{2} \int_{\Omega}\left(\rho_{0}\left|\mathbf{u}_{0}\right|^{2}+\left|\nabla \mathbf{d}_{0}\right|^{2}\right) d \mathbf{x} .
\end{aligned}
$$

Proof. Integrating (5.5) over the time interval $[0, t]$, we obtain the energy equality (6.1).

Now, we proceed to prove weak-strong uniqueness. Let $(\tilde{\rho}, \tilde{\mathbf{u}}, \Pi, \tilde{\mathbf{d}})$ be a global (in time) weak solution. On one hand, as the density $\tilde{\rho}$ satisfies

$$
\left\{\begin{array}{l}
\partial_{t} \tilde{\rho}+\tilde{\mathbf{u}} \cdot \nabla \tilde{\rho}=0 \\
\left.\tilde{\rho}\right|_{t=0}=\rho_{0} \in W^{1, r}(\Omega)
\end{array}\right.
$$

with $\tilde{\mathbf{u}} \in\left(L_{\text {loc }}^{2}\left(\mathbb{R}^{+} ; H_{0}^{1}(\Omega)\right)\right)^{3}$, Theorem 1 in $[9]$ insures that $\tilde{\rho} \in C\left(\mathbb{R}^{+} ; W^{1, r^{-}}(\Omega)\right)$ for all $r^{-}<r$. On the other hand, we remark that, in view of the regularity of the 
strong solution $(\rho, \mathbf{u}, P, \mathbf{d})$, we deduce from the weak formulation that

$$
\begin{aligned}
& \int_{\Omega} \tilde{\rho} \tilde{\mathbf{u}} \cdot \mathbf{u} d \mathbf{x}+\int_{0}^{t} \int_{\Omega} \nabla \tilde{\mathbf{u}}: \nabla \mathbf{u} d \mathbf{x} d \tau \\
& =\int_{\Omega} \rho_{0}\left|\mathbf{u}_{0}\right|^{2} d \mathbf{x}+\int_{0}^{t} \int_{\Omega} \tilde{\rho} \tilde{\mathbf{u}} \cdot\left(\partial_{\tau} \mathbf{u}+\tilde{\mathbf{u}} \cdot \nabla \mathbf{u}\right) d \mathbf{x} d \tau-\int_{0}^{t} \int_{\Omega}(\nabla \tilde{\mathbf{d}})^{\top} \triangle \tilde{\mathbf{d}} \cdot \mathbf{u} d \mathbf{x} d \tau
\end{aligned}
$$

and

$$
\begin{aligned}
& \int_{\Omega} \nabla \tilde{\mathbf{d}}: \nabla \mathbf{d} d \mathbf{x}-\int_{\Omega}\left|\nabla \mathbf{d}_{0}\right|^{2} d \mathbf{x} \\
& =\int_{0}^{t} \int_{\Omega}\left(-\tilde{\mathbf{d}} \cdot \triangle \mathbf{d}_{\tau}+\tilde{\mathbf{u}} \cdot \nabla \tilde{\mathbf{d}} \cdot \Delta \mathbf{d}-\triangle \tilde{\mathbf{d}} \cdot \Delta \mathbf{d}-|\nabla \tilde{\mathbf{d}}|^{2} \tilde{\mathbf{d}} \cdot \triangle \mathbf{d}\right) d \mathbf{x} d \tau
\end{aligned}
$$

for almost all $t \in\left(0, T_{0}\right)$.

If we write

$$
\begin{aligned}
& \tilde{\rho} \partial_{t} \mathbf{u}+\tilde{\rho} \tilde{\mathbf{u}} \cdot \nabla \mathbf{u}-\triangle \mathbf{u}+\nabla P \\
& =(\tilde{\rho}-\rho)\left(\partial_{t} \mathbf{u}+\mathbf{u} \cdot \nabla \mathbf{u}\right)+\tilde{\rho}(\tilde{\mathbf{u}}-\mathbf{u}) \cdot \nabla \mathbf{u}-\nabla\left(\frac{|\nabla \mathbf{d}|^{2}}{2}\right)-(\nabla \mathbf{d})^{\top} \triangle \mathbf{d}
\end{aligned}
$$

then multiply (6.4) by $\tilde{\mathbf{u}}$ and integrate over $\Omega \times(0, t)$ to find

$$
\begin{aligned}
& \int_{0}^{t} \int_{\Omega}\left(\tilde{\rho} \partial_{\tau} \mathbf{u}+\tilde{\rho} \tilde{\mathbf{u}} \cdot \nabla \mathbf{u}\right) \cdot \tilde{\mathbf{u}} d \mathbf{x} d \tau+\int_{0}^{t} \int_{\Omega} \nabla \mathbf{u}: \nabla \tilde{\mathbf{u}} d \mathbf{x} d \tau \\
& =\int_{0}^{t} \int_{\Omega}\left((\tilde{\rho}-\rho)\left(\partial_{\tau} \mathbf{u}+\mathbf{u} \cdot \nabla \mathbf{u}\right) \cdot \tilde{\mathbf{u}}+\tilde{\rho}(\tilde{\mathbf{u}}-\mathbf{u}) \cdot \nabla \mathbf{u} \cdot \tilde{\mathbf{u}}-(\nabla \mathbf{d})^{\top} \triangle \mathbf{d} \cdot \tilde{\mathbf{u}}\right) d \mathbf{x} d \tau
\end{aligned}
$$

then meanwhile replace $\partial_{\tau} \mathbf{d}$ by (1.1c) in $(\underline{6.3)}$ to get

$$
\begin{aligned}
& \int_{\Omega} \nabla \tilde{\mathbf{d}}: \nabla \mathbf{d} d \mathbf{x}-\int_{\Omega}\left|\nabla \mathbf{d}_{0}\right|^{2} d \mathbf{x} \\
&=\int_{0}^{t} \int_{\Omega}\left(-2 \triangle \tilde{\mathbf{d}} \cdot \triangle \mathbf{d}+\mathbf{u} \cdot \nabla \mathbf{d} \cdot \triangle \tilde{\mathbf{d}}+\tilde{\mathbf{u}} \cdot \nabla \tilde{\mathbf{d}} \cdot \triangle \mathbf{d}-|\nabla \mathbf{d}|^{2} \mathbf{d} \cdot \triangle \tilde{\mathbf{d}}\right. \\
&\left.-|\nabla \tilde{\mathbf{d}}|^{2} \tilde{\mathbf{d}} \cdot \triangle \mathbf{d}\right) d \mathbf{x} d \tau
\end{aligned}
$$

Combining (6.2), (6.5) and (6.6), we get for almost all $t \in\left(0, T_{0}\right)$,

$$
\begin{aligned}
& \int_{\Omega}(\tilde{\rho} \tilde{\mathbf{u}} \cdot \mathbf{u}+\nabla \tilde{\mathbf{d}}: \nabla \mathbf{d}) d \mathbf{x}+2 \int_{0}^{t} \int_{\Omega}(\nabla \tilde{\mathbf{u}}: \nabla \mathbf{u}+\triangle \tilde{\mathbf{d}} \cdot \triangle \mathbf{d}) d \mathbf{x} d \tau \\
& =\int_{\Omega}\left(\rho_{0}\left|\mathbf{u}_{0}\right|^{2}+\left|\nabla \mathbf{d}_{0}\right|^{2}\right) d \mathbf{x}-\int_{0}^{t} \int_{\Omega}(\nabla \tilde{\mathbf{d}})^{\top} \triangle \tilde{\mathbf{d}} \cdot \mathbf{u} d \mathbf{x} d \tau \\
& \quad+\int_{0}^{t} \int_{\Omega}\left((\tilde{\rho}-\rho)\left(\partial_{\tau} \mathbf{u}+\mathbf{u} \cdot \nabla \mathbf{u}\right) \cdot \tilde{\mathbf{u}}+\tilde{\rho}(\tilde{\mathbf{u}}-\mathbf{u}) \cdot \nabla \mathbf{u} \cdot \tilde{\mathbf{u}}-(\nabla \mathbf{d})^{\top} \triangle \mathbf{d} \cdot \tilde{\mathbf{u}}\right) d \mathbf{x} d \tau \\
& \quad+\int_{0}^{t} \int_{\Omega}\left(\mathbf{u} \cdot \nabla \mathbf{d} \cdot \triangle \tilde{\mathbf{d}}+\tilde{\mathbf{u}} \cdot \nabla \tilde{\mathbf{d}} \cdot \triangle \mathbf{d}-|\nabla \mathbf{d}|^{2} \mathbf{d} \cdot \triangle \tilde{\mathbf{d}}-|\nabla \tilde{\mathbf{d}}|^{2} \tilde{\mathbf{d}} \cdot \triangle \mathbf{d}\right) d \mathbf{x} d \tau .
\end{aligned}
$$


From (6.4) and (1.1c), using the same argument we used to get the energy estimate (6.1), we get for almost all $t \in\left(0, T_{0}\right)$,

$$
\begin{aligned}
& \frac{1}{2} \int_{\Omega}\left(\tilde{\rho}|\mathbf{u}|^{2}+|\nabla \mathbf{d}|^{2}\right) d \mathbf{x}+\int_{0}^{t} \int_{\Omega}\left(|\nabla \mathbf{u}|^{2}+\left.\left.|\triangle \mathbf{d}+| \nabla \mathbf{d}\right|^{2} \mathbf{d}\right|^{2}\right) d \mathbf{x} d \tau \\
& =\frac{1}{2} \int_{\Omega}\left(\rho_{0}\left|\mathbf{u}_{0}\right|^{2}+\left|\nabla \mathbf{d}_{0}\right|^{2}\right) d \mathbf{x} \\
& \quad+\int_{0}^{t} \int_{\Omega}\left((\tilde{\rho}-\rho)\left(\partial_{\tau} \mathbf{u}+\mathbf{u} \cdot \nabla \mathbf{u}\right) \cdot \mathbf{u}+\tilde{\rho}(\tilde{\mathbf{u}}-\mathbf{u}) \cdot \nabla \mathbf{u} \cdot \mathbf{u}\right) d \mathbf{x} d \tau
\end{aligned}
$$

Here we have used the fact that $\partial_{t} \tilde{\rho}=-\tilde{\mathbf{u}} \cdot \nabla \tilde{\rho}$.

Then, adding (2.1) and (6.8) and substracting (6.7), together with the fact that (5.6) and $(\tilde{\rho}, \tilde{\mathbf{u}}, \Pi, \tilde{\mathbf{d}})$ is a weak solution, we obtain

$$
\begin{gathered}
\frac{1}{2} \int_{\Omega}\left(\tilde{\rho}|\mathbf{u}-\tilde{\mathbf{u}}|^{2}+|\nabla \mathbf{d}-\nabla \tilde{\mathbf{d}}|^{2}\right) d \mathbf{x}+\int_{0}^{t} \int_{\Omega}\left(|\nabla \mathbf{u}-\nabla \tilde{\mathbf{u}}|^{2}+|\triangle \mathbf{d}-\triangle \tilde{\mathbf{d}}|^{2}\right) d \mathbf{x} d \tau \\
\leq \int_{0}^{t} \int_{\Omega}\left((\tilde{\rho}-\rho)\left(\partial_{\tau} \mathbf{u}+\mathbf{u} \cdot \nabla \mathbf{u}\right) \cdot(\mathbf{u}-\tilde{\mathbf{u}})-\tilde{\rho}(\mathbf{u}-\tilde{\mathbf{u}}) \cdot \nabla \mathbf{u} \cdot(\mathbf{u}-\tilde{\mathbf{u}})\right) d \mathbf{x} d \tau \\
\quad-\int_{0}^{t} \int_{\Omega}((\nabla \mathbf{d}-\nabla \tilde{\mathbf{d}}) \cdot \triangle \mathbf{d} \cdot(\mathbf{u}-\tilde{\mathbf{u}})-\mathbf{u} \cdot(\nabla \mathbf{d}-\nabla \tilde{\mathbf{d}}) \cdot(\triangle \mathbf{d}-\triangle \tilde{\mathbf{d}}) \\
\left.+\left(|\nabla \mathbf{d}|^{2} \mathbf{d}-|\nabla \tilde{\mathbf{d}}|^{2} \tilde{\mathbf{d}}\right) \cdot(\triangle \mathbf{d}-\triangle \tilde{\mathbf{d}})\right) d \mathbf{x} d \tau
\end{gathered}
$$

Hence, for almost all $t \in\left(0, T_{0}\right)$ and for all $\varepsilon>0$, we have

$$
\begin{aligned}
& \frac{1}{2} \int_{\Omega}\left(\tilde{\rho}|\mathbf{u}-\tilde{\mathbf{u}}|^{2}+|\nabla \mathbf{d}-\nabla \tilde{\mathbf{d}}|^{2}\right) d \mathbf{x}+\int_{0}^{t} \int_{\Omega}\left(|\nabla \mathbf{u}-\nabla \tilde{\mathbf{u}}|^{2}+|\triangle \mathbf{d}-\triangle \tilde{\mathbf{d}}|^{2}\right) d \mathbf{x} d \tau \\
& \leq \int_{0}^{t}\left(C_{\varepsilon}\left\|\partial_{\tau} \mathbf{u}+\mathbf{u} \cdot \nabla \mathbf{u}\right\|_{L^{3}}^{2}\|\rho-\tilde{\rho}\|_{L^{2}}^{2}\right. \\
& \left.\quad+\varepsilon\|\mathbf{u}-\tilde{\mathbf{u}}\|_{L^{6}}^{2}\right) d \tau+\varepsilon \int_{0}^{t} \int_{\Omega}|\triangle \mathbf{d}-\triangle \tilde{\mathbf{d}}|^{2} d \mathbf{x} d \tau \\
& \quad+C_{\varepsilon} \int_{0}^{t}\left(\|\nabla \mathbf{d}\|_{L^{\infty}}^{4} \int_{\Omega}|\mathbf{d}-\tilde{\mathbf{d}}|^{2} d \mathbf{x}\right) d \tau+\int_{0}^{t}\left(\|\nabla \mathbf{u}\|_{L^{\infty}} \int_{\Omega} \tilde{\rho}|\mathbf{u}-\tilde{\mathbf{u}}|^{2} d \mathbf{x}\right) d \tau \\
& \quad+\int_{0}^{t}\left(C_{\varepsilon}\left(\|\nabla \mathbf{d}+\nabla \tilde{\mathbf{d}}\|_{L^{\infty}}^{2}+\|\mathbf{u}\|_{L^{\infty}}^{2}+\|\triangle \mathbf{d}\|_{L^{3}}^{2}\right) \int_{\Omega}|\nabla \mathbf{d}-\nabla \tilde{\mathbf{d}}|^{2} d \mathbf{x}\right) d \tau
\end{aligned}
$$

Here we have used Hölder's inequality and Cauchy's inequality with $\varepsilon$.

Now, we wish to estimate $\|\rho-\tilde{\rho}\|_{L^{2}}$ and $\|\mathbf{d}-\tilde{\mathbf{d}}\|_{L^{2}}$. We write

$$
\partial_{t}(\rho-\tilde{\rho})+\nabla(\rho-\tilde{\rho}) \cdot \tilde{\mathbf{u}}=(\tilde{\mathbf{u}}-\mathbf{u}) \cdot \nabla \rho
$$

and

$(6.11) \partial_{t}(\mathbf{d}-\tilde{\mathbf{d}})+\mathbf{u} \cdot \nabla(\mathbf{d}-\tilde{\mathbf{d}})+(\mathbf{u}-\tilde{\mathbf{u}}) \cdot \nabla \tilde{\mathbf{d}}=\Delta \mathbf{d}-\triangle \tilde{\mathbf{d}}+|\nabla \mathbf{d}|^{2} \mathbf{d}-|\nabla \tilde{\mathbf{d}}|^{2} \tilde{\mathbf{d}}$

Multiplying (6.10) (resp. (6.11) $)$ by $\rho-\tilde{\rho}($ resp. $\mathbf{d}-\tilde{\mathbf{d}})$ and integrating over $\Omega \times(0, t)$, we have

$$
\frac{1}{2} \int_{\Omega}|\rho-\tilde{\rho}|^{2} d \mathbf{x}=\int_{0}^{t} \int_{\Omega}(\rho-\tilde{\rho})(\tilde{\mathbf{u}}-\mathbf{u}) \cdot \nabla \rho d \mathbf{x} d \tau
$$


and

$$
\begin{aligned}
& \frac{1}{2} \int_{\Omega}|\mathbf{d}-\tilde{\mathbf{d}}|^{2} d \mathbf{x} \\
& =-\int_{0}^{t} \int_{\Omega}(\mathbf{u}-\tilde{\mathbf{u}}) \cdot \nabla \tilde{\mathbf{d}} \cdot(\mathbf{d}-\tilde{\mathbf{d}}) d \mathbf{x} d \tau-\int_{0}^{t} \int_{\Omega}|\nabla \mathbf{d}-\nabla \tilde{\mathbf{d}}|^{2} d \mathbf{x} d \tau \\
& \quad+\int_{0}^{t} \int_{\Omega}|\nabla \mathbf{d}|^{2}|\mathbf{d}-\tilde{\mathbf{d}}|^{2} d \mathbf{x} d \tau+\int_{0}^{t} \int_{\Omega}(\nabla \mathbf{d}+\nabla \tilde{\mathbf{d}}):(\nabla \mathbf{d}-\nabla \tilde{\mathbf{d}}) \tilde{\mathbf{d}} \cdot(\mathbf{d}-\tilde{\mathbf{d}}) d \mathbf{x} d \tau .
\end{aligned}
$$

Employing the same argument as in (6.9), we get

$$
\frac{1}{2} \int_{\Omega}|\rho-\tilde{\rho}|^{2} d \mathbf{x} \leq \int_{0}^{t}\left(C_{\varepsilon}\|\nabla \rho\|_{L^{3}}^{2}\|\rho-\tilde{\rho}\|_{L^{2}}^{2}+\varepsilon\|\mathbf{u}-\tilde{\mathbf{u}}\|_{L^{6}}^{2}\right) d \tau,
$$

$$
\begin{aligned}
& \frac{1}{2} \int_{\Omega}|\mathbf{d}-\tilde{\mathbf{d}}|^{2} d \mathbf{x} \\
& \leq \int_{0}^{t}\left(C_{\varepsilon}\|\nabla \tilde{\mathbf{d}}\|_{L^{3}}^{2}+\|\nabla \mathbf{d}\|_{L^{\infty}}^{2}+\frac{1}{2}\right) \int_{\Omega}|\mathbf{d}-\tilde{\mathbf{d}}|^{2} d \mathbf{x} d \tau+\varepsilon \int_{0}^{t}\|\mathbf{u}-\tilde{\mathbf{u}}\|_{L^{6}}^{2} d \tau \\
& \quad+\int_{0}^{t}\left(1+\frac{\|\nabla \mathbf{d}+\nabla \tilde{\mathbf{d}}\|_{L^{\infty}}^{2}}{2}\right) \int_{\Omega}|\nabla \mathbf{d}-\nabla \tilde{\mathbf{d}}|^{2} d \mathbf{x} d \tau
\end{aligned}
$$

Using Sobolev's inequality $\|\mathbf{u}-\tilde{\mathbf{u}}\|_{L^{6}} \leq C\|\nabla \mathbf{u}-\nabla \tilde{\mathbf{u}}\|_{L^{2}}$, we eventually get from (6.9), (6.12) and (6.13), for almost all $t \in\left(0, T_{0}\right)$,

$$
\begin{aligned}
& \int_{\Omega}\left(\tilde{\rho}|\mathbf{u}-\tilde{\mathbf{u}}|^{2}+|\nabla \mathbf{d}-\nabla \tilde{\mathbf{d}}|^{2}+|\rho-\tilde{\rho}|^{2}+|\mathbf{d}-\tilde{\mathbf{d}}|^{2}\right) d \mathbf{x} \\
& +\int_{0}^{t} \int_{\Omega}\left(|\nabla \mathbf{u}-\nabla \tilde{\mathbf{u}}|^{2}+|\triangle \mathbf{d}-\triangle \tilde{\mathbf{d}}|^{2}\right) d \mathbf{x} d \tau \\
& \leq \int_{0}^{t} \int_{\Omega}\left(C_{\varepsilon}(\tau)\left(|\rho-\tilde{\rho}|^{2}+|\nabla \mathbf{d}-\nabla \tilde{\mathbf{d}}|^{2}+|\mathbf{d}-\tilde{\mathbf{d}}|^{2}\right)\right. \\
& \left.\quad+C(\tau)\left(\tilde{\rho}|\mathbf{u}-\tilde{\mathbf{u}}|^{2}+|\nabla \mathbf{d}-\nabla \tilde{\mathbf{d}}|^{2}+|\mathbf{d}-\tilde{\mathbf{d}}|^{2}\right)\right) d \mathbf{x} d \tau
\end{aligned}
$$

where $C_{\varepsilon}(\cdot), C(\cdot)$ denote various nonnegative measurable functions in $L^{1}\left(0, T_{0}\right)$ which occurred when we applied the parabolic type estimates for quasi-linear equations (cf. 24, Chapter VI, Section 2) to (1.1c) to obtain $\tilde{\mathbf{d}}(\cdot, t) \in C^{1, \alpha}$ for some $\alpha>0$, with the $C^{1, \alpha}$ norm independent of $t$. We hence conclude that $\mathbf{u}=\tilde{\mathbf{u}}, \mathbf{d}=\tilde{\mathbf{d}}$ and $\rho=\tilde{\rho}$ a.e. in $\Omega \times\left(0, T_{0}\right)$ by applying Grönwall's inequality.

The proof of Theorem 2.2 is complete.

\section{REFERENCES}

[1] Herbert Amann, Linear and quasilinear parabolic problems. Vol. I, Abstract linear theory, Monographs in Mathematics, vol. 89, Birkhäuser Boston Inc., Boston, MA, 1995. MR 1345385 (96g:34088)

[2] Jöran Bergh and Jörgen Löfström, Interpolation spaces. An introduction, Grundlehren der Mathematischen Wissenschaften, No. 223, Springer-Verlag, Berlin, 1976. MR0482275 (58 \#2349)

[3] L. Caffarelli, R. Kohn, and L. Nirenberg, Partial regularity of suitable weak solutions of the Navier-Stokes equations, Comm. Pure Appl. Math. 35 (1982), no. 6, 771-831, DOI 10.1002/cpa.3160350604. MR673830 (84m:35097)

[4] S. Chandrasekhar, Liquid crystals. 2nd ed., Cambridge University Press, 1992. 
[5] Blanca Climent-Ezquerra, Francisco Guillén-González, and Marko Rojas-Medar, Reproductivity for a nematic liquid crystal model, Z. Angew. Math. Phys. 57 (2006), no. 6, 984-998, DOI 10.1007/s00033-005-0038-1. MR2279252(2008e:76004)

[6] Daniel Coutand and Steve Shkoller, Well-posedness of the full Ericksen-Leslie model of nematic liquid crystals (English, with English and French summaries), C. R. Acad. Sci. Paris Sér. I Math. 333 (2001), no. 10, 919-924, DOI 10.1016/S0764-4442(01)02161-9. MR.1873808 (2002j:82118)

[7] R. Danchin, Global existence in critical spaces for compressible Navier-Stokes equations, Invent. Math. 141 (2000), no. 3, 579-614, DOI 10.1007/s002220000078. MR.1779621 (2003a:76090)

[8] R. Danchin, Density-dependent incompressible fluids in bounded domains, J. Math. Fluid Mech. 8 (2006), no. 3, 333-381, DOI 10.1007/s00021-004-0147-1. MR2258416 (2007k:76032)

[9] Benoit Desjardins, Linear transport equations with initial values in Sobolev spaces and application to the Navier-Stokes equations, Differential Integral Equations 10 (1997), no. 3, 577-586. MR 1744862 (2000k:35234)

[10] Shijin Ding, Junyu Lin, Changyou Wang, and Huanyao Wen, Compressible hydrodynamic flow of liquid crystals in 1-D, Discrete Contin. Dyn. Syst. 32 (2012), no. 2, 539-563, DOI 10.3934/dcds.2012.32.539. MR2837072(2012j:35329)

[11] Shijin Ding, Changyou Wang, and Huanyao Wen, Weak solution to compressible hydrodynamic flow of liquid crystals in dimension one, Discrete Contin. Dyn. Syst. Ser. B 15 (2011), no. 2, 357-371, DOI 10.3934/dcdsb.2011.15.357. MR2754089(2012a:35237)

[12] J. L. Ericksen, Hydrostatic theory of liquid crystals, Arch. Rational Mech. Anal. 9 (1962), 371-378. MR0137403 (25 \#855)

[13] Benoît Desjardins, Regularity of weak solutions of the compressible isentropic NavierStokes equations, Comm. Partial Differential Equations 22 (1997), no. 5-6, 977-1008, DOI 10.1080/03605309708821291. MR1452175 (98e:35126)

[14] J. L. Ericksen, Conservation laws for liquid crystals, Trans. Soc. Rheology 5 (1961), 23-34. MR.0158610 (28 \#1833)

[15] J. L. Ericksen, Continuum theory of nematic liquid crystals. Res. Mechanica 21 (1987), 381392.

[16] Hiroshi Fujita and Tosio Kato, On the Navier-Stokes initial value problem. I, Arch. Rational Mech. Anal. 16 (1964), 269-315. MR0166499(29 \#3774)

[17] Giovanni P. Galdi, An introduction to the mathematical theory of the Navier-Stokes equations. Vol. I, Linearized steady problems, Springer Tracts in Natural Philosophy, vol. 38, Springer-Verlag, New York, 1994. MR1284205(95i:35216a)

[18] Robert Hardt and David Kinderlehrer, Mathematical questions of liquid crystal theory, Theory and applications of liquid crystals (Minneapolis, Minn., 1985), IMA Vol. Math. Appl., vol. 5, Springer, New York, 1987, pp. 151-184, DOI 10.1007/978-1-4613-8743-5_9. MR.900833 (88m:82033)

[19] Robert Hardt, David Kinderlehrer, and Fang-Hua Lin, Existence and partial regularity of static liquid crystal configurations, Comm. Math. Phys. 105 (1986), no. 4, 547-570. MR.852090 (88a:35207)

[20] Xianpeng $\mathrm{Hu}$ and Dehua Wang, Global solution to the three-dimensional incompressible flow of liquid crystals, Comm. Math. Phys. 296 (2010), no. 3, 861-880, DOI 10.1007/s00220-0101017-8. MR2628824 (2011c:76014)

[21] Tao Huang, Changyou Wang, and Huanyao Wen, Strong solutions of the compressible nematic liquid crystal flow, J. Differential Equations 252 (2012), no. 3, 2222-2265, DOI 10.1016/j.jde.2011.07.036. MR2860617 (2012k:35410)

[22] Tao Huang, Changyou Wang, and Huanyao Wen, Blow up criterion for compressible nematic liquid crystal flows in dimension three, Arch. Ration. Mech. Anal. 204 (2012), no. 1, 285-311, DOI 10.1007/s00205-011-0476-1. MR2898742

[23] Fei Jiang and Zhong Tan, Global weak solution to the flow of liquid crystals system, Math. Methods Appl. Sci. 32 (2009), no. 17, 2243-2266, DOI 10.1002/mma.1132. MR2561116 (2010m:35392)

[24] O. A. Ladyženskaja, V. A. Solonnikov, and N. N. Ural'ceva, Linear and quasilinear equations of parabolic type, (Russian) translated from the Russian by S. Smith. Translations of Mathematical Monographs, Vol. 23, American Mathematical Society, Providence, R.I., 1968. MR.0241822(39 \#3159b) 
[25] F. M. Leslie, Some constitutive equations for liquid crystals, Arch. Rational Mech. Anal. 28 (1968), no. 4, 265-283, DOI 10.1007/BF00251810. MR1553506

[26] Xiaoli Li and Dehua Wang, Global solution to the incompressible flow of liquid crystals, J. Differential Equations 252 (2012), no. 1, 745-767, DOI 10.1016/j.jde.2011.08.045. MR2852225 (2012k:35419)

[27] Fang-Hua Lin, Nonlinear theory of defects in nematic liquid crystals; phase transition and flow phenomena, Comm. Pure Appl. Math. 42 (1989), no. 6, 789-814, DOI 10.1002/cpa.3160420605. MR 1003435 (90g:82076)

[28] Fang-Hua Lin and Chun Liu, Nonparabolic dissipative systems modeling the flow of liquid crystals, Comm. Pure Appl. Math. 48 (1995), no. 5, 501-537, DOI 10.1002/cpa.3160480503. MR $1329830(96 \mathrm{a}: 35154)$

[29] Fang-Hua Lin and Chun Liu, Partial regularity of the dynamic system modeling the flow of liquid crystals, Discrete Contin. Dynam. Systems 2 (1996), no. 1, 1-22. MR.1367385 (96m:35255)

[30] Fang-Hua Lin and Chun Liu, Existence of solutions for the Ericksen-Leslie system, Arch. Ration. Mech. Anal. 154 (2000), no. 2, 135-156, DOI 10.1007/s002050000102. MR.1784963 (2003a:76014)

[31] Fanghua Lin and Chun Liu, Static and dynamic theories of liquid crystals, J. Partial Differential Equations 14 (2001), no. 4, 289-330. MR1883167(2003b:82063)

[32] Fanghua Lin, Junyu Lin, and Changyou Wang, Liquid crystal flows in two dimensions, Arch. Ration. Mech. Anal. 197 (2010), no. 1, 297-336, DOI 10.1007/s00205-009-0278-x. MR2646822(2011c:35411)

[33] Pierre-Louis Lions, Mathematical topics in fluid mechanics. Vol. 1, Incompressible models, Oxford Science Publications, Oxford Lecture Series in Mathematics and its Applications, vol. 3, The Clarendon Press, Oxford University Press, New York, 1996. MR1422251 (98b:76001)

[34] Chun Liu and Noel J. Walkington, Approximation of liquid crystal flows, SIAM J. Numer. Anal. 37 (2000), no. 3, 725-741 (electronic), DOI 10.1137/S0036142997327282. MR 1740379 (2000k:65174)

[35] Lan Ming Liu and Xian Gao Liu, A blow-up criterion for strong solutions to the compressible liquid crystals system (Chinese, with English and Chinese summaries), Chinese Ann. Math. Ser. A 32 (2011), no. 4, 393-406. MR2884827 (2012k:35423)

[36] X. Liu, L. Liu, and Y. Hao, Existence of strong solutions for the compressible Ericksen-Leslie model. Preprint, 2011.

[37] Xian-Gao Liu and Jie Qing, Globally weak solutions to the flow of compressible liquid crystals system, Discrete Contin. Dyn. Syst. 33 (2013), no. 2, 757-788, DOI 10.3934/dcds.2013.33.757. MR2975133

[38] Yuming Qin and Lan Huang, Global existence and regularity of a $1 D$ liquid crystal system, Nonlinear Anal. Real World Appl. 15 (2014), 172-186, DOI 10.1016/j.nonrwa.2013.07.003. MR 3110563

[39] Huan Sun and Chun Liu, On energetic variational approaches in modeling the nematic liquid crystal flows, Discrete Contin. Dyn. Syst. 23 (2009), no. 1-2, 455-475, DOI 10.3934/dcds.2009.23.455. MR2449088 (2009h:76064)

[40] Dehua Wang and Cheng Yu, Global weak solution and large-time behavior for the compressible flow of liquid crystals, Arch. Ration. Mech. Anal. 204 (2012), no. 3, 881-915, DOI 10.1007/s00205-011-0488-x. MR2917124

Institute of Applied Physics and Computational Mathematics, Beijing 100088, PeoPLE's Republic of ChinA

Current address: College of Science, Beijing University of Posts and Telecommunications,

Beijing, 100876, People's Republic of China

E-mail address: xlli@bupt.edu.cn

Department of Mathematics, University of Pittsburgh, Pittsburgh, Pennsylvania 15260

E-mail address: dwang@math.pitt.edu 